\title{
Racing Against The Clock: Evidence-Based Vs. Time-Based Decisions
}

\author{
Guy E. Hawkins ${ }^{1} \&$ Andrew Heathcote ${ }^{2}$ \\ 1: School of Psychology, University of Newcastle, Australia \\ 2: School of Psychology, University of Tasmania, Australia
}

\begin{abstract}
Classical dynamic theories of decision making assume that responses are triggered by accumulating a threshold amount of information. Recently, there has been a growing appreciation that the passage of time also plays a role in triggering responses. We propose that decision processes are composed of two diffusive accumulation mechanisms - one evidence-based and one timebased - that compete in an independent race architecture. We show that this Timed Racing Diffusion Model (TRDM) provides a unified, comprehensive and quantitatively accurate explanation of key decision phenomenaincluding the effects of implicit and explicit deadlines and the relative speed of correct and error responses under speed-accuracy tradeoffs - without requiring additional mechanisms that have been criticized as being ad-hoc in theoretical motivation and difficult to estimate, such as trial-to-trial variability parameters, collapsing thresholds, or urgency signals. In contrast, our addition is grounded in a widely validated account of time-estimation performance, enabling the same mechanism to simultaneously account for interval estimation and decision making with an explicit deadline.
\end{abstract}

Keywords: Decision making; Time estimation; Cognitive model; Response time.

Funding: This work was supported by: Australian Research Council (ARC) Discovery Early Career Researcher Award (Hawkins, DE170100177); ARC Discovery Project (Hawkins, DP180103613); ARC Discovery Project (Heathcote, DP160101891); Visiting Professor Grant, University of Amsterdam (Heathcote). The funding sources had no role in the study design; in the collection, analysis and interpretation of data; in the writing of the report; and in the decision to submit the article for publication. Declarations of interest: none. Earlier versions of this work were posted online at psyarxiv.com/m4uh7 and presented at the 2019 meetings of the Society for Mathematical Psychology and the Psychonomic Society. 


\section{Introduction}

We propose and test a new model of dynamic decision making, the Timed Racing Diffusion Model (TRDM). TRDM unifies major information-controlled phenomena, such as the speed-accuracy tradeoff, and time-controlled phenomena, such as interval timing and decision making in the presence of external and internal deadlines. Literatures about these phenomena have evolved somewhat independently yet have considerable parallels. In particular, most of the major theories of speeded decision making assume a latent process of accumulating evidence (e.g., Brown \& Heathcote, 2008; Ratcliff, 1978; Usher \& McClelland, 2001; Van Zandt, Colonius, \& Proctor, 2000), while major theories of time estimation assume a latent process of accumulating from a constant input (e.g., Merchant, Harrington, \& Meck, 2013; Simen, Balci, deSouza, Cohen, \& Holmes, 2011). Although modern timeestimation models are based on a simple diffusion process with a constant mean drift rate within a trial, decision models have diverged from similarly simple roots (Stone, 1960). This increase in complexity occurred because trial-to-trial variability in the drift rate and the starting point of evidence accumulation (Laming, 1968; Ratcliff, 1978) are required to account for differences in the speed of error and correct responses (e.g., Ratcliff \& Rouder, 1998). Indeed, trial-to-trial variability has even been suggested to dominate diffusive effects (e.g., Brown \& Heathcote, 2005, 2008; Heathcote \& Love, 2012).

The TRDM is inspired by Simen and colleagues' work that integrates the diffusion decision model with timing accumulation (Balci \& Simen, 2014, 2016; Simen, Vlasov, \& Papadakis, 2016). It differs in having a race architecture consisting only of simple diffusion processes. We demonstrate that the two cognitive activities - decision making and timing - have considerable overlap. Although our focus here is mainly on explicit decision-making behavior, we note that the timing component of our model can operate independently and inherits the ability of a simple diffusion process to provide a neurally plausible account for benchmark phenomena in time estimation (e.g., time-scale invariance, see Simen et al., 2016).

A core commitment of the TRDM is that both evidence accumulation and an internal sense of time both contribute to decision making, although the influence of the latter component may vary. Previous domain-specific theories have proposed a related role for timing. For example, in the lexical decision task it has been proposed that if the stimulus is not recognized as a word by a participant-determined 'internal deadline' a non-word response is made (Coltheart, Rastle, Perry, Langdon, \& Ziegler, 2001; Grainger \& Jacobs, 1996). Similar theories exist in the recognition memory and short-term memory scanning literatures, where an item is determined to be "new" (i.e., not been previously studied) when an internal deadline elapses prior to an evidence-based "old" response reaching threshold (Diller, Nobel, \& Shiffrin, 2001; Nobel \& Shiffrin, 2001; Nosofsky, Little, Donkin, \& Fific, 2011). Terminating visual search has also been modeled as a 'time to quit' signal: each inspected element of the display not containing the target contributes toward a dynamically accumulated signal that is compared to a 'quitting time' threshold, producing a target-absent response when crossed (Shi, Allenmark, Zhu, Elliott, \& Müller, 2020; Wolfe \& Van Wert, 2010). Paradigms where a decision can be made to defer making a choice have been modeled with an evidence accumulation mechanism racing against an accumulator driven by a constant input; if the latter accumulator reaches threshold first then the choice is deferred 
(Bhatia \& Mullett, 2016).

Previous domain-general theories of choice have also hinted at the importance of elapsed time as a trigger to terminate a decision process independent of the current state of accumulated evidence. For example, in Audley's (1960) 'runs' model the most uncertain of responses were suggested to be subject to an internal stopping time - the maximum time participants are willing to wait to make a decision. In simple detection, the 'deadline' model was proposed with a time estimation process under the decision maker's control, so it can be strategically calibrated to the timing properties of a task to ensure efficient detection (Ollman \& Billington, 1972).

More recent theories have argued for an indirect effect of time through evidence thresholds that decrease as accumulation proceeds (i.e., "collapsing thresholds", Bowman, Kording, \& Gottfried, 2012; Drugowitsch, Moreno-Bote, Churchland, Shadlen, \& Pouget, 2012; Hawkins, Forstmann, Wagenmakers, Ratcliff, \& Brown, 2015; Milosavljevic, Malmaud, Huth, Koch, \& Rangel, 2010; Shadlen \& Kiani, 2013; Tajima, Drugowitsch, \& Pouget, 2016). These theories assume that taking account of elapsed decision time is critical so the decision maker does not waste effort on difficult decisions where continued evidence integration is unlikely to be helpful. It has even been suggested that an entirely evidenceindependent signal is integrated (i.e., an "urgency signal" or "urgency gating", Churchland, Kiani, \& Shadlen, 2008; Cisek, Puskas, \& El-Murr, 2009; Ditterich, 2006). The TRDM can also avoid wasteful evidence integration but remains committed to the idea that decisions are primarily based on relevant evidence. Importantly, its account of the influence of time is directly testable because the TRDM provides an explicit process-level model of timing.

After describing the TRDM in detail in the next section, Part 1 of the paper explores its ability to explain benchmark phenomena in information- and time-controlled decisionmaking paradigms. We compare its performance with the major theories that have either championed the necessity of adding trial-to-trial parameter variability to a core diffusive process, the Diffusion Decision Model (DDM; Ratcliff, 1978; Ratcliff, Smith, Brown, \& McKoon, 2016), or assume the effects of diffusive variability can be entirely neglected because they are dominated by trial-to-trial variability, the Linear Ballistic Accumulator (LBA; Brown \& Heathcote, 2008). We demonstrate that, despite its broader explanatory reach, the TRDM provides an equal or better account than the DDM and LBA in terms of goodness of fit, both descriptively and after an adjustment for differences in model flexibility. We also show that this account can have different psychological implications because of the effects of timer-based responding on accuracy.

In the paradigms examined in Part 1 of this paper the stimuli associated with each choice occurred with equal probability, and we assumed equi-probable response guessing for timer-based responses. In Part 2 of the paper we fit the TRDM to a paradigm with unequal stimulus probabilities (Wagenmakers, Ratcliff, Gomez, \& McKoon, 2008) and explore different mechanisms by which timer-based responses can take stimulus probabilities into account. We then examine what happens when inhomogeneous stimuli mapped to the same response are likely to cause trial-to-trial evidence differences. Our analysis suggests these effects are largely absorbed into TRDM's diffusion coefficient (i.e., its index of the level of moment-to-moment diffusive variability) with little effect on other parameters. Hence, trial-to-trial variability can modify inferences based on diffusion-coefficient estimates, but does not tend to compromise the interpretation of other parameters. 
In Part 3 of the paper we explore the timing aspects of the TRDM in more detail. We first fit it to data from the sustained attention to response task (SART; Robertson, Manly, Andrade, Baddeley, \& Yiend, 1997; Smallwood et al., 2004; Smallwood \& Schooler, 2006) whose repetitive nature produces a mixture of more strongly evidence-based decisions and rhythmic, time-based responses that cannot be accounted for by conventional evidence-accumulation models. We compare the fit of the TRDM to Hawkins, Mittner, Forstmann, and Heathcote's (2019) paradigm-specific model, but as in Part 1 our main aim is to demonstrate an adequate fit and a coherent and more general explanation compared to this paradigm-specific model. Finally, we validated the timing component of the TRDM in a paradigm where the same participants estimated temporal intervals and made choices (Miletić \& Van Maanen, 2019). The full TRDM provided an excellent explanation of individual choice performance, and its timing component did the same for interval production. Most importantly, convergent validity was supported by a clear correlation between individual differences in the precision of the timing processes in each task.

\section{The Timed Racing Diffusion Model (TRDM)}

Figure 1 provides a schematic overview of the model's structure. It assumes a race between evidence-based and time-based processes. There is a separate response accumulator for each response option, such that evidence for each of $N$ response options is represented as a race to threshold between $N$ evidence accumulators. Each evidence accumulator is a one-boundary diffusive process drifting to a threshold, which produces a Wald or inverse Gaussian distribution of finishing times. We assume this evidence process races against a timing process, also represented as a one-boundary diffusive process, which has precedent in the timing literature (e.g., Simen et al., 2011). The timing process has its own stimulusindependent rate at which it drifts to its timing threshold. The two processes $-N$ oneboundary diffusion processes representing the accumulation of evidence and a single oneboundary diffusion process representing an internal timer - race against each other. The decision terminates when either of the two latent processes crosses its respective boundary. The predicted response time is the time taken for the first accumulator to cross threshold, plus an offset for stimulus encoding and motor production times. The predicted choice is a function of which accumulator crossed threshold: the $N$ evidence accumulators have a one-to-one mapping with the response options, and the timing accumulator leads to an equiprobable guess between the $N$ response options. We refer to this collective set of modeling assumptions as the Timed Racing Diffusion Model, or TRDM.

The set of racing diffusion processes provides a neurally plausible implementation of decision and timing processes. Neural activation in the form of populations of excitatory and inhibitory spike counts are often modeled as Poisson, where the difference between weighted spike counts from both populations approximates the expected tendency to drift to a positive threshold in a one-boundary diffusion process. Almost identical theoretical motivations of this form have appeared in the literatures on time estimation (e.g., Simen et al., 2011) and evidence accumulation (e.g., Smith, 2010).

\section{Race Architecture}

A response can be generated in two ways: 


\section{Evidence Process}

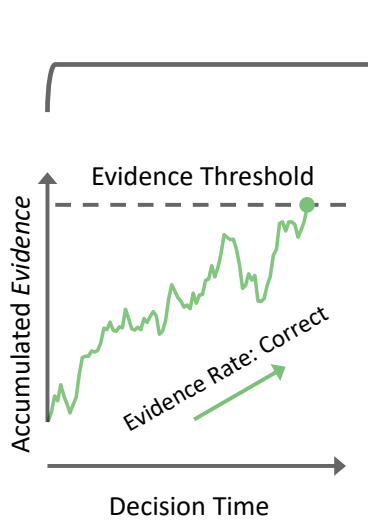

人

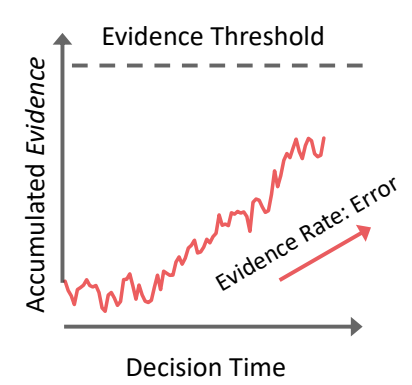

Timing Process
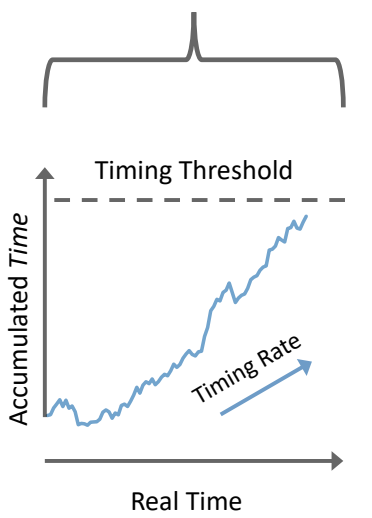

Figure 1. Schematic of the Timed Racing Diffusion Model (TRDM). The TRDM proposes that decisions are composed of two latent racing processes: an evidence process composed of $N$ evidence accumulators for a decision between $N$ options (in this example, green for the correct response and red for the incorrect response), and a timing process composed of a single timing accumulator (blue). The first accumulator to reach threshold gives the decision outcome and time. See text for details.

1. An accumulator in the evidence process crosses its evidence threshold before the timing process crosses its timing threshold. The predicted response corresponds to the evidence threshold that was crossed.

2. The timing process crosses its timing threshold before any accumulator in the evidence process crosses an evidence threshold. The predicted response is a stimulusuninformed guess.

To specify the TRDM we denote the density functions for each of the $N$ evidence accumulation nodes at time $t$ as $f_{E, n}(t)$, where $n \in\{1, \ldots, N\}$, with corresponding distribution functions $F_{E, n}(t)$. Similarly, we denote the density and distribution functions of the timing process as $f_{T}(t)$ and $F_{T}(t)$, respectively. The probability density function (PDF) for a response of option 1 at time $t$ is given by

$$
P D F_{1}(t)=f_{E, 1}(t)\left(\prod_{n=2}^{N} 1-F_{E, n}(t)\right)\left(1-F_{T}(t)\right)+g_{T, 1} \cdot f_{T}(t)\left(\prod_{n=1}^{N} 1-F_{E, n}(t)\right)
$$

where $g_{T, 1}$ is the probability that a stimulus-uninformed guess favors option 1 . Throughout we assume that $g_{T, n}=\frac{1}{N}$ for all $n$. More complicated forms for $g_{T}$ are possible; for example, $g_{T}$ could be informed by the state of the evidence accumulation process at time $t$, conditioned on the evidence process not terminating prior to $t$ ("partial information", see Ratcliff, 1980, 1988, 2006). We did not find these more complex assumptions to be necessary in the data analyzed in Part 1 of the paper, and we explore this assumption in detail in Part 2 of the paper. 


\section{Distribution Functions}

A one-boundary diffusion process results in times to reach the boundary that follow a Wald or inverse Gaussian distribution. This process drifts with rate $\gamma>0$ and diffusion coefficient $\sigma>0$ until it crosses threshold $\alpha>0$. When applied to response time (RT) data, there is an additional non-zero onset time of the diffusion process and a time to produce a motor response once a choice is made. These combined times are often referred to as the non-decision time, $\tau>0$.

The density and distribution functions for the one-boundary diffusion process are (Heathcote, 2004)

$$
f(t \mid \gamma, \sigma, \alpha, \tau)=\frac{\alpha}{\sigma \sqrt{2 \pi(t-\tau)^{3}}} \exp \left(-\frac{[\alpha-\gamma(t-\tau)]^{2}}{2 \sigma^{2}(t-\tau)}\right)
$$

and

$$
F(t \mid \gamma, \sigma, \alpha, \tau)=\Phi\left(\frac{\gamma(t-\tau)-\alpha}{\sigma \sqrt{t-\tau}}\right)+\exp \left(\frac{2 \alpha \gamma}{\sigma^{2}}\right) \cdot \Phi\left(-\frac{\gamma(t-\tau)+\alpha}{\sigma \sqrt{t-\tau}}\right),
$$

where $\Phi$ is the standard normal distribution function. In order to identify the model we must fix at least one parameter value for an accumulation process (Donkin, Brown, \& Heathcote, 2009); here, we set the value of the response threshold to $\alpha=1$ in the evidence and timing processes. This setting makes it easy to compare the speed and variability of the evidence accumulation and timing processes (e.g., whether the evidence process is more variable than the timing process). For clarity, we add subscript $E$ to refer to parameters of the evidence accumulation process $\left(\gamma_{E}, \sigma_{E}, \tau_{E}\right)$ and subscript $T$ to refer to parameters of the timing process $\left(\gamma_{T}, \sigma_{T}, \tau_{T}\right)$.

Evidence Accumulation Process. The parameters of the one-boundary diffusion process have well-studied interpretations in terms of evidence accumulation. The drift rate $\left(\gamma_{E}\right)$ represents the average speed with which information is extracted from the stimulus. The diffusion coefficient $\left(\sigma_{E}\right)$ represents the moment-to-moment variability in the information accumulation process. The non-decision time $\left(\tau_{E}\right)$ is a non-zero onset time that represents the combined time required to encode the stimulus and produce a motor response.

An important feature of the TRDM is that we do not assume across-trial variability in any parameters of the evidence accumulation process. The assumption of trial-to-trial variability in drift rate and the starting point of evidence accumulation (Laming, 1968; Ratcliff, 1978; Ratcliff \& Rouder, 1998), and in non-decision time (Ratcliff, Van Zandt, \& McKoon, 1999), has become standard in modern implementations of the DDM (e.g., Ratcliff \& McKoon, 2008; Ratcliff et al., 2016). Some or all of these types of variability have also been incorporated as standard in other race models such as the LBA (Brown \& Heathcote, 2008) and lognormal race (Heathcote \& Love, 2012), and even other forms of racing diffusion models (Logan, Van Zandt, Verbruggen, \& Wagenmakers, 2014; Tillman, Van Zandt, \& Logan, in press), though not all models (Usher \& McClelland, 2001).

Nevertheless, the specific assumptions about across-trial variability in some decision parameters has been criticized as lacking theoretical motivation (Jones \& Dzhafarov, 2014; for alternative perspectives see Heathcote, Wagenmakers, \& Brown, 2014; Smith, Ratcliff, 
\& McKoon, 2014). We argue that a key theoretical advantage of the TRDM is that all model parameters represent a theoretical commitment to a cognitive process; there are no across-trial variability parameters and no ad hoc distributional assumptions. We show that the TRDM achieves this without sacrificing goodness-of-fit to data. We note that this is a simplifying assumption. We do not claim that trial-to-trial variability in parameters does not exist. Rather, we assume that in the data sets we examine this form of variability has little or no explanatory role. Later, we examine how TRDM estimates are affected if substantial trial-to-trial variability is present.

Timing Process. The parameters of the one-boundary diffusion process also have sensible interpretations as a timing process. The drift rate $\left(\gamma_{T}\right)$ represents the rate at which perceived time clocks over, like an internal counter. The diffusion coefficient $\left(\sigma_{T}\right)$ represents the variability in the timing accumulation process. The shift parameter $\left(\tau_{T}\right)$ represents the onset of the timing process, which need not necessarily commence at trial onset or be equivalent to the corresponding shift parameter of the evidence process (i.e., $\left.\tau_{E}\right)$, although these and other assumptions may be plausible in some circumstances.

\section{Parameterization}

Drift Rates. For a choice between two options - the context we consider here the standard race model approach is to directly estimate drift rates $\gamma_{E, 1}$ and $\gamma_{E, 2}$ that drive accumulation processes racing toward response thresholds $\alpha_{E, 1}$ and $\alpha_{E, 2}$, respectively; the race is unbiased when $\alpha_{E, 1}=\alpha_{E, 2}$, simplifying to a common threshold $\alpha_{E}$. Here, we estimate two alternative parameters: the mean speed or rate of the evidence process $\left(\gamma_{E, \mu}\right)$ and the sensitivity to stimulus information $\left(\gamma_{E, \zeta}\right)$. The mean and sensitivity parameters are transformed to drift rates for options 1 and 2 by $\gamma_{E, 1}=\gamma_{E, \mu}+\frac{\gamma_{E, \zeta}}{2}$ and $\gamma_{E, 2}=\gamma_{E, \mu}-\frac{\gamma_{E, \zeta}}{2}$. As with the standard parameterization of drift rates in a race model, the mean-sensitivity scheme presents a range of possible parameterizations for choices between $N>2$ options. Some of these parameterizations will be more suitable in some contexts than others; we leave it to future research to empirically test them.

The mean-sensitivity estimation scheme is simply a re-parameterization of the standard estimation scheme of 'correct' vs. 'error' (or 'match' vs. 'mismatch') drift rates in a race model. It is analogous to Strickland, Loft, Remington, and Heathcote's (2018) reparameterization in terms of the quantity (mean) and quality (sensitivity) of LBA accumulation rates (see also Boag, Strickland, Loft, \& Heathcote, 2019, for the relationship of this re-parameterization to divisive normalization). This re-parameterization is not essential to the TRDM's goodness of fit but we believe it has some interpretation advantages. First, the sensitivity parameter $\left(\gamma_{E, \zeta}\right)$ represents the difference in evidence between two options and thus reflects the discriminability of the stimulus information. It can be interpreted in a similar manner to the sensitivity parameter in signal detection theory $\left(d^{\prime}\right)$ or the mean drift rate in the DDM. Larger values of $\gamma_{E, \zeta}$ indicate greater discriminability and lead to a higher proportion of correct responses. Second, the mean speed of the evidence process indicates the overall rate at which all evidence accumulation nodes drift toward the threshold. This is largely unrelated, though not independent of, stimulus identity, so we refer to this as a generic 'drive' or urgency to make a response. This drive might differ based on contextual factors such as task instructions to emphasize decision speed or accuracy (e.g., Rae, Heathcote, Donkin, Averell, \& Brown, 2014). The drive parameter $\left(\gamma_{E, \mu}\right)$ can also be 
compared to the drift rate of the timing process $\left(\gamma_{T}\right)$, permitting inference on a common scale about the overall rate at which the evidence and timing processes drift toward their respective thresholds.

Thresholds. In a similar vein, in order to quantify reponse bias, we parameterize the mean evidence required to trigger a response $\left(\alpha_{E, \mu}\right)$ and the difference in evidence required for the two options $\left(\alpha_{E, \zeta}\right)$, again such that $\alpha_{E, 1}=\alpha_{E, \mu}+\frac{\alpha_{E, \zeta}}{2}$ and $\alpha_{E, 2}=\alpha_{E, \mu}-$ $\frac{\alpha_{E, \zeta}}{2}$ (see Garton, Reynolds, Hinder, \& Heathcote, 2019, for a related approach with the LBA). In the contexts we consider below that assume a single evidence threshold across all conditions, $\alpha_{E, \mu}=1$ to satisfy the scaling condition. Where there is more than one evidence threshold across conditions, we set the evidence threshold for one condition to a fixed value $\left(\alpha_{E, \mu, 1}=1\right.$; typically a speed-emphasis condition $)$ and freely estimate the mean evidence threshold in the remaining $k-1$ conditions. The difference parameter $\left(\alpha_{E, \zeta}\right)$ is similar to the start point parameter in the DDM, allowing for systematic response biases (e.g., a stimulus manipulation with frequent vs. infrequent response categories) and/or random biases (e.g., individual differences in propensity to respond with one option over another).

Diffusion Coefficient. The diffusion coefficient is freely estimated from data, allowing a direct comparison of the variability in the evidence accumulation process and the timing process. This allows us to address psychologically interesting questions, for example, do people more precisely track the passing of time $\left(\sigma_{T}\right)$ or the integration of action-relevant information $\left(\sigma_{E}\right)$.

TRDM Parameterization Constraints. Taken together, for a two-choice task we estimate seven base parameters for the TRDM: $\gamma_{E, \mu}, \gamma_{E, \zeta}, \alpha_{E, \zeta}, \sigma_{E}, \tau_{E}, \gamma_{T}$, and $\sigma_{T}$. For all experimental designs analyzed here we impose various parameter constraints across conditions, with each design-specific constraint discussed further below with the corresponding experiment. One common constraint we assume across all applications is a fixed value of the onset time of the timing process at $\tau_{T}=.05 \mathrm{~s}$. This implements our theoretical assumption that the timing process is unconditionally and rapidly triggered following trial onset. This value also balances differences across the tasks and data sets we consider. Some tasks used pre-stimulus intervals with no variance such that $\tau_{T}=0 \mathrm{~s}$ is plausible; that is, participants could perfectly predict the onset time of the stimulus and thus initiate their timer at stimulus onset. Other tasks had variable pre-stimulus intervals such that stimulus onset time was less predictable and thus $\tau_{T}=0 \mathrm{~s}$ may be less plausible. We chose to emphasize uniformity in the fixed value across applications. Nevertheless, we are not committed to this particular value of the onset time for the timing process and note that future applications of the TRDM may adjust $\tau_{T}$ to suit the requirements of the task under consideration or the prior expectations of the researchers. In Part 3 we present one such application with strong motivation for $\tau_{T}=0 \mathrm{~s}$.

From a practical perspective, we arrived at the constraint on $\tau_{T}$ following preliminary analyses estimating both shift parameters $\left(\tau_{E}, \tau_{T}\right)$ that always resulted in $\tau_{T}<\tau_{E}$, though also generally led to poorer convergence of our estimation procedure and poor parameter recovery compared to a fixed value of $\tau_{T}$. We conducted a sensitivity analysis by assuming different fixed values for $\tau_{T}$. Full details are reported in the supplementary material. In summary, none of our model comparison outcomes are dependent on a particular fixed value of $\tau_{T}$. The main consideration is that $\tau_{T}<\tau_{E}$. By setting $\tau_{T}=.05 \mathrm{~s}$ we reduced the number of freely estimated base parameters and greatly improved parameter identifiability. 


\section{Qualitative Predictions}

Figure 2 displays qualitative predictions of the TRDM in order to provide intuition about the model's scope. Each row shows the effect of shifting the row-named parameter from a low value (on the left) to a high value (on the right), with all other parameters held constant. Many of the predicted parameter effects are qualitatively similar to existing evidence accumulation models; that is, the TRDM nests many conventional explanations for decision making phenomena. For instance, as sensitivity to stimulus evidence increases the predicted behavioral effect is a large increase in accuracy, and slightly faster correct RT and slower errors; this is the expected outcome of a stimulus-based manipulation of choice difficulty. Similarly, when more evidence is accumulated prior to responding, accuracy increases while the RT distribution changes in predictable ways: mean RT becomes slower, variance increases, and skew decreases; this is the classic explanation of the speed-accuracy tradeoff.

Nevertheless, the predictions for some parameters of the TRDM's evidence process are not shared with other evidence accumulation models. For all existing models, as nondecision time increases the onset of the RT distribution is delayed but no other features of RT or accuracy change. ${ }^{1}$ In contrast, in the TRDM there is an increasing proportion of trials terminated by the timer as non-decision time increases. This can generate a nonnegligible combination of behavioral outcomes: lower accuracy and a shift from slower errors to faster errors because the timing process terminates an increasing proportion of trials with a random guess. An increasing proportion of timing-terminated vs. evidence-terminated processes can also produce a shallower leading edge when the two processes have different onset times.

The TRDM generates novel predictions from the two latent components of the timing process that are freely estimated from data (lower two rows of Figure 2). When the timing rate increases the timing process reaches threshold earlier, on average, and so the expected proportion of timing-terminated decisions relative to evidence-terminated decisions increases. This has the behavioral effect of speeding responses and reducing accuracy. The skew of the RT distribution is also reduced. This is because the faster mean speed of the timing process censors the slowest evidence processes, leading to fewer predicted slow responses. In fact, as the timing rate increases the predicted RT distributions become increasingly Gaussian. This is a feature of the TRDM that allows it to explain Gaussian-like RT distributions that have, to date, been most effectively explained with collapsing threshold models. We show that the TRDM provides an alternative explanation of such results in Part 1 of the paper.

To understand the effect of moment-to-moment variability in the timing process we first consider the predicted distribution of a single accumulator. As the moment-to-moment variability of a one-boundary diffusion process increases the variance in the distribution of finishing times also increases, including a faster leading edge and slower tail. In the context of the multi-accumulator race architecture of the TRDM, increased variability in the timing process leads to many very slow random variates from the timing process that will never terminate the decision; they are censored by the earlier-terminating evidence process.

\footnotetext{
${ }^{1}$ Similarly to the DDM (Ratcliff, Gomez, \& McKoon, 2004), we note that across-trial variability in nondecision time generates a shallower leading edge of the RT distribution.
} 


\section{Evidence Process}

Rate $\left(\gamma_{\mathrm{E}, \mu}\right)$
Lower accuracy
Faster RT
Decreasing variance
Decreasing skew
Sensitivity $\left(\gamma_{\mathrm{E}, \zeta}\right)$
Higher accuracy
Faster correct RT
Slower error RT

Threshold $\left(\alpha_{\mathrm{E}, \mu}\right)$
Higher accuracy
Slower RT
Increasing variance
Decreasing skew

Variability $\left(\sigma_{\mathrm{E}}\right)$
Lower accuracy
Faster RT
Increasing skew
Slower errors become faster errors
Shallower leading edge
Lower accuracy
Non-Dision Time $\left(\tau_{\mathrm{E}}\right)$
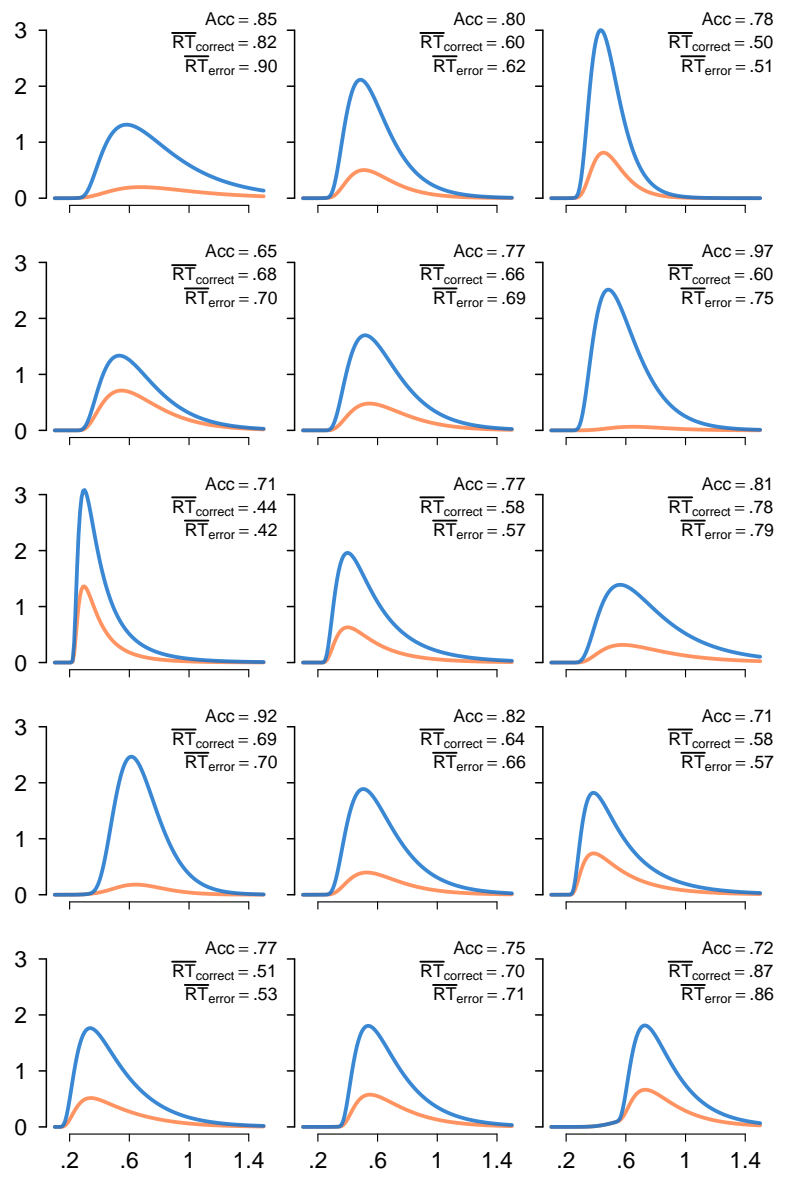

\section{Timing Process}

Rate $\left(\gamma_{\mathrm{T}}\right)$
Lower accuracy
Faster RT
Decreasing skew

Variability $\left(\sigma_{\mathrm{T}}\right)$
Lower accuracy
Faster errors
Shallower leading edge
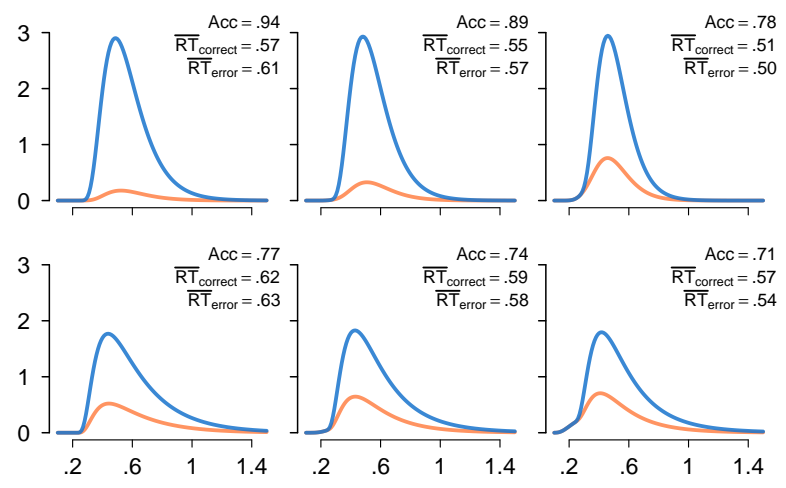

Response Time (s)

Figure 2. Qualitative predictions of the TRDM. Each row focuses on a different model parameter. The three columns show changes in the predicted RT distributions for correct (blue) and error (orange) responses as the row-named parameter shifts from a small to large value with all other parameters held at fixed values. The upper right of each panel shows the accuracy and mean RT for correct and error responses for the cell. The left column provides a qualitative description of the predicted changes as the row-named parameter shifts from lower to higher values. 
However, large variability of the timing process also generates a small proportion of random variates are very fast; they terminate before the evidence process reaches threshold, and can even terminate before onset of the evidence process (i.e., faster than $\tau_{E}$ ). This pattern of fast-terminating timing processes generates the predictions in Figure 2: lower accuracy, faster errors, and a shallower leading edge. We explore the critical role of timing variability in Part 3 of the paper.

\section{Estimation and Model Comparison}

We used a hierarchical Bayesian framework to simultaneously estimate the TRDM parameters at the participant and group levels. We assumed mildly informative prior distributions for the group-level parameters, which allowed the estimated parameters to vary across a range of plausible values. Appendix A has complete details of the prior distributions and parameter estimation routines. The density and distribution functions of the TRDM were implemented in the $\mathrm{R}$ statistical environment based on a generalization of shifted Wald functions developed by Trisha Van Zandt. Code for applying the TRDM to all data sets considered in this paper is available at osf.io/5cf $7 \mathrm{w}$, including an implementation of the TRDM in the R-based DMC for Bayesian estimation of a variety of different evidence-accumulation models (Heathcote, Lin, et al., 2019).

In the applications in Part 1 we compared the TRDM's account of the data relative to three existing models. The first model is a racing diffusion model without a timing process (RDM; Logan et al., 2014; Tillman et al., in press). The RDM was specified, parameterized, and estimated exactly as described for the evidence accumulation process of the TRDM. This tests the necessity of the latent timing process. ${ }^{2}$ The second model is a widely applied member of the class of independent race models, the LBA (Brown \& Heathcote, 2008). This compares the TRDM to a benchmark race model that relies entirely on trial-to-trial parameter variability. The final model is the widely applied 'full' diffusion model (DDM) with across-trial variability parameters for drift rate, start point, and nondecision time (Ratcliff \& Tuerlinckx, 2002; Ratcliff et al., 1999). This compares the TRDM to a benchmark relative-evidence accumulation model. Appendix A outlines the estimation scheme for the comparison models and Appendix B outlines model parameterizations for all data sets. We used the Deviance Information Criterion (DIC; Spiegelhalter, Best, Carlin, \& van der Linde, 2002) to compare models in a way that takes account of model flexibility both in terms of the number of estimated parameters (parametric complexity) and the way in which those parameters interact (functional-form complexity). The model with the lowest DIC is preferred.

\section{Part 1: Information-Controlled and Time-Controlled Choice Phenomena}

\section{Information-Controlled Choice: Decisions Under Time Pressure}

The speed-accuracy tradeoff caused by time pressure has traditionally been understood to be the result of strategically adjusting the quantity of evidence required to trigger a

\footnotetext{
${ }^{2}$ Tillman et al. (in press) developed and tested a version of the RDM that included across-trial variability in the starting point of evidence accumulation. We did not compare the TRDM to the RDM with acrosstrial variability as it would not be informative to our goal of assessing the necessity of the latent timing mechanism of the TRDM relative to a non-timing racing diffusion model.
} 
decision by changing the average distance from the starting point of evidence accumulation to the evidence threshold (e.g., Heitz, 2014; Ratcliff \& Rouder, 1998). In addition, more recent work has suggested that time pressure might also change the speed of evidence accumulation and non-decision time (e.g., Dutilh et al., 2018; Rae et al., 2014; Spieser, Servant, Hasbroucq, \& Burle, 2017; Starns, Pazzaglia, Rotello, Hautus, \& Macmillan, 2013). Here, we demonstrate that the TRDM provides an accurate account of speed-accuracy tradeoff data through an additional process: participants strategically adapting the amount of time they are willing to commit to a decision by adjusting their 'clock speed' (i.e., the drift rate of the timing accumulator, $\left.\gamma_{T}\right) .{ }^{3}$ Changes in clock speed reproduce the qualitative trends of the speed-accuracy tradeoff: a faster timing rate generates quicker responses that are less accurate, on average, since the timing process randomly selects a response (Figure 2).

We comprehensively compared the set of four model types (TRDM, LBA, RDM and DDM) and the different combinations of mechanisms they can employ to explain the speedaccuracy tradeoff. For each type of model we fit variants that either fixed or allowed to vary freely as a function of the time pressure manipulation the parameters that mediate the different mechanisms (see Appendix B for complete details). For the TRDM, this involved estimating all combinations of effects of the time pressure manipulation on mean evidence thresholds, mean evidence drift rate, evidence sensitivity, non-decision time, and the drift rate of the timing process. We omitted the null model where no parameters varied, producing a set of $2^{5}-1=31 \mathrm{TRDM}$ variants. Our motivation for this exploratory test of the TRDM is that it is the first use of the model so we did not wish to exclude any a priori plausible explanation of the speed-accuracy tradeoff, including parameterizations that did not vary evidence thresholds with the time pressure manipulation. It is an open question whether a TRDM with a timing rate effect but without an evidence threshold and/or evidence rate effect is able to quantitatively capture the trends in speed-accuracy tradeoff data.

For the LBA, RDM and DDM we assumed mean evidence thresholds always differed as a function of the time pressure manipulation owing to the overwhelming evidence for this effect in the literature. For the LBA and RDM, we then estimated all combinations of mean evidence drift rate, evidence sensitivity and non-decision time, producing a set of $2^{3}=8$ LBAs and RDMs. For the DDM, which does not have any equivalent to the mean evidence rate in the other racing accumulator models, we estimated all combinations of non-decision time and evidence sensitivity, producing a set of 4 DDMs. Taken together then, we compared 51 different models. We used DIC as our primary method of model comparison in order take account of differences in the flexibility of the different models, but we also graphically compare the best models on their descriptive adequacy in order to identify any qualitative patterns of misfit.

Data. We applied the TRDM to two well-studied speed-accuracy tradeoff data sets. We first provide a brief overview of each data set followed by a combined presentation of the results. We refer the reader to the primary sources for in-depth descriptions of experimental details. Model estimation details are provided in appendices.

Forstmann et al. (2008) tested 19 participants on a perceptual random-dot-motion task. A random-dot-motion decision is based on a cloud of dynamically moving dots, of

\footnotetext{
${ }^{3}$ Alternatively, the rate may be constant and the timing threshold adjusted $\left(\alpha_{T}\right)$. Given appropriate changes in the diffusion coefficient these two approaches have identical effects.
} 
which a certain percentage move coherently to the left or right of the display, with the remaining dots moving randomly. The participant's task was to identify the direction of coherent motion. They made decisions at a single motion coherence level $(60 \%)$ with a pseudo-randomized cue presented prior to each trial indicating the level of time pressure in the upcoming trial: fast, accurate, or neutral. Participants received feedback consistent with the condition following each decision and completed 840 trials that were evenly split across the three levels of time pressure.

Wagenmakers et al. (2008), Experiment 1, tested 17 participants in a lexical decision task. Each participant made decisions about words of high, low or very low frequency, and pronouncable non-words; throughout we denote these stimulus conditions HF, LF, VLF and NW, respectively. Each of the four word types appeared an equal number of times within each of the 20 blocks of trials. The blocks alternated between speed- and accuracy-emphasis instructions, with feedback consistent with the current emphasis condition delivered following each trial. Participants completed 1920 trials in total. Consistent with Wagenmakers et al. (2008), we estimated separate evidence sensitivity parameters for each stimulus condition.

Model Comparison Results. Tables 1 and 2 show DICs for the best performing parameterization of each model type; Appendix C provides DICs for the complete set of 51 models though we summarize key results here. For both data sets the TRDM performed best, with the DDM the second best for the perceptual-decision data and the LBA second best for the lexical-decision data. For the TRDM, effects on timing and evidence rates had the strongest support in both data sets in the sense of being most often included in models with better (i.e., lower) DIC values. For lexical decisions the best TRDM model (i.e., the model with the smallest DIC) included all five mechanisms. Among these mechanisms the weakest contribution was made by the evidence-threshold effect, followed by evidence sensitivity, so that the models dropping one or other of these effects still out-performed all variants of the best competitor model type, the LBA. For perceptual decisions the effect on evidence sensitivity was dropped in the best TRDM. Three other TRDM variants - with all five effects, dropping the mean evidence rate effect, or dropping evidence sensitivity and non-decision time effects - also outperformed all variants of the best competitor model type, the DDM. These results provide clear support for the TRDM's novel timer mechanism as playing an important role in explaining the speed-accuracy tradeoff.

Table 1

DIC model comparison for perceptual decisions (Forstmann et al., 2008). Rows show models. Columns 2-6 indicate whether a parameter was freely estimated across the levels of the time pressure manipulation $(\checkmark)$ or constrained to a single value (blank; - indicates a parameter does not exist in a model). Column 7 shows the number of model parameters freely estimated for each participant. Column 8 shows DICs zero-referenced to the DIC-preferred model such that positive values indicate a poorer explanation of the data. Rows are ordered by DIC.

\begin{tabular}{lccccccc} 
Model & $\begin{array}{c}\text { Evidence } \\
\text { Threshold }\end{array}$ & $\begin{array}{c}\text { Evidence } \\
\text { Rate }\end{array}$ & $\begin{array}{c}\text { Evidence } \\
\text { Sensitivity }\end{array}$ & $\begin{array}{c}\text { Evidence } \\
\text { NDT }\end{array}$ & $\begin{array}{c}\text { Timing } \\
\text { Rate }\end{array}$ & $\begin{array}{c}\text { Number of } \\
\text { Free Parameters }\end{array}$ & $\Delta$ DIC \\
\hline TRDM & $\checkmark$ & $\checkmark$ & & $\checkmark$ & $\checkmark$ & 15 & 0 \\
DDM & $\checkmark$ & - & & $\checkmark$ & - & 11 & 118 \\
LBA & $\checkmark$ & $\checkmark$ & $\checkmark$ & & - & 13 & 472 \\
RDM & $\checkmark$ & $\checkmark$ & $\checkmark$ & $\checkmark$ & - & 13 & 621
\end{tabular}


Table 2

DIC model comparison for lexical decisions (Wagenmakers et al., 2008). All details are as described in Table 1.

\begin{tabular}{lccccccc} 
Model & $\begin{array}{c}\text { Evidence } \\
\text { Threshold }\end{array}$ & $\begin{array}{c}\text { Evidence } \\
\text { Rate }\end{array}$ & $\begin{array}{c}\text { Evidence } \\
\text { Sensitivity }\end{array}$ & $\begin{array}{c}\text { Evidence } \\
\text { NDT }\end{array}$ & $\begin{array}{c}\text { Timing } \\
\text { Rate }\end{array}$ & $\begin{array}{c}\text { Number of } \\
\text { Free Parameters }\end{array}$ & $\Delta$ DIC \\
\hline TRDM & $\checkmark$ & $\checkmark$ & $\checkmark$ & $\checkmark$ & $\checkmark$ & 18 & 0 \\
LBA & $\checkmark$ & $\checkmark$ & $\checkmark$ & $\checkmark$ & - & 17 & 175 \\
DDM & $\checkmark$ & - & $\checkmark$ & $\checkmark$ & - & 16 & 1,022 \\
RDM & $\checkmark$ & $\checkmark$ & $\checkmark$ & $\checkmark$ & - & 15 & 2,358
\end{tabular}

Goodness of Fit. The DIC advantage for the best TRDM was 118 units relative to the best DDM for perceptual decisions and 175 units relative to the best LBA for lexical decisions. These differences are very substantial, corresponding to negligible model weights (Wagenmakers \& Farrell, 2004) for the best competitor models $\left(\ll 10^{-20}\right)$. However, the correction for complexity used by DIC rests on assumptions that may be questioned. Further, DIC does not address absolute fit, so the best model may still miss important features of the data. Hence, we now examine goodness of fit, graphically comparing the descriptive adequacy of the best TRDM model with the best competitor models.

Figure 3 shows observed vs posterior predicted data for individual participants, separately for the TRDM and the best-performing competitor models in Tables 1 and 2. Each symbol represents the RT data and posterior prediction from a single condition from a single participant in each experiment (columns), separately for accuracy and quantiles of the correct and error RT distributions (rows).

The TRDM and both competitor models precisely explained all qualitative trends in the data, and many of the quantitative trends including accuracy and median correct and error RTs. There were some minor discrepancies between the competitor models and data that were not shared by the TRDM, such as the DDM underpredicting the $10^{t h}$ percentile for correct RTs for perceptual decisions (dots tend to fall below the diagonal), and the LBA overpredicting accuracy for the lexical decisions (dots tend to fall above the diagonal). These discrepancies are evident in differences in a measure of absolute fit - root-meansquare-error (RMSE) - accompanying each panel in the plot. Otherwise the models are relatively comparable in terms of RMSE, with a slight advantage for the TRDM.

Overall, the TRDMs descriptive account was both qualitatively and quantitatively accurate and equivalent to or better than the best-performing competitor model for the perceptual and lexical decision data sets. This was the case even though the best-performing competitor models were different for each data set, suggesting the TRDM might provide a more encompassing explanation of the speed-accuracy tradeoff.

Parameter Estimates. Although DIC may select an effect as being present that does not mean that it is necessarily important in explaining the average magnitude of the time pressure effect (e.g., an effect may be selected in order to explain individual differences). We now examine parameter estimates for the best TRDM model in order understand the psychological implications of their explanation of time-pressure effects. We also demonstrate that it is valid to interpret these estimates because the TRDM is a measurement model in the sense that its parameters can be recovered from data simulated following the two designs of the two experiments (Heathcote, Brown, \& Wagenmakers, 2015).

Tables 3 and 4 show the posterior median and $95 \%$ credible interval (CI) of the group- 


\section{Perceptual Decisions Lexical Decisions}
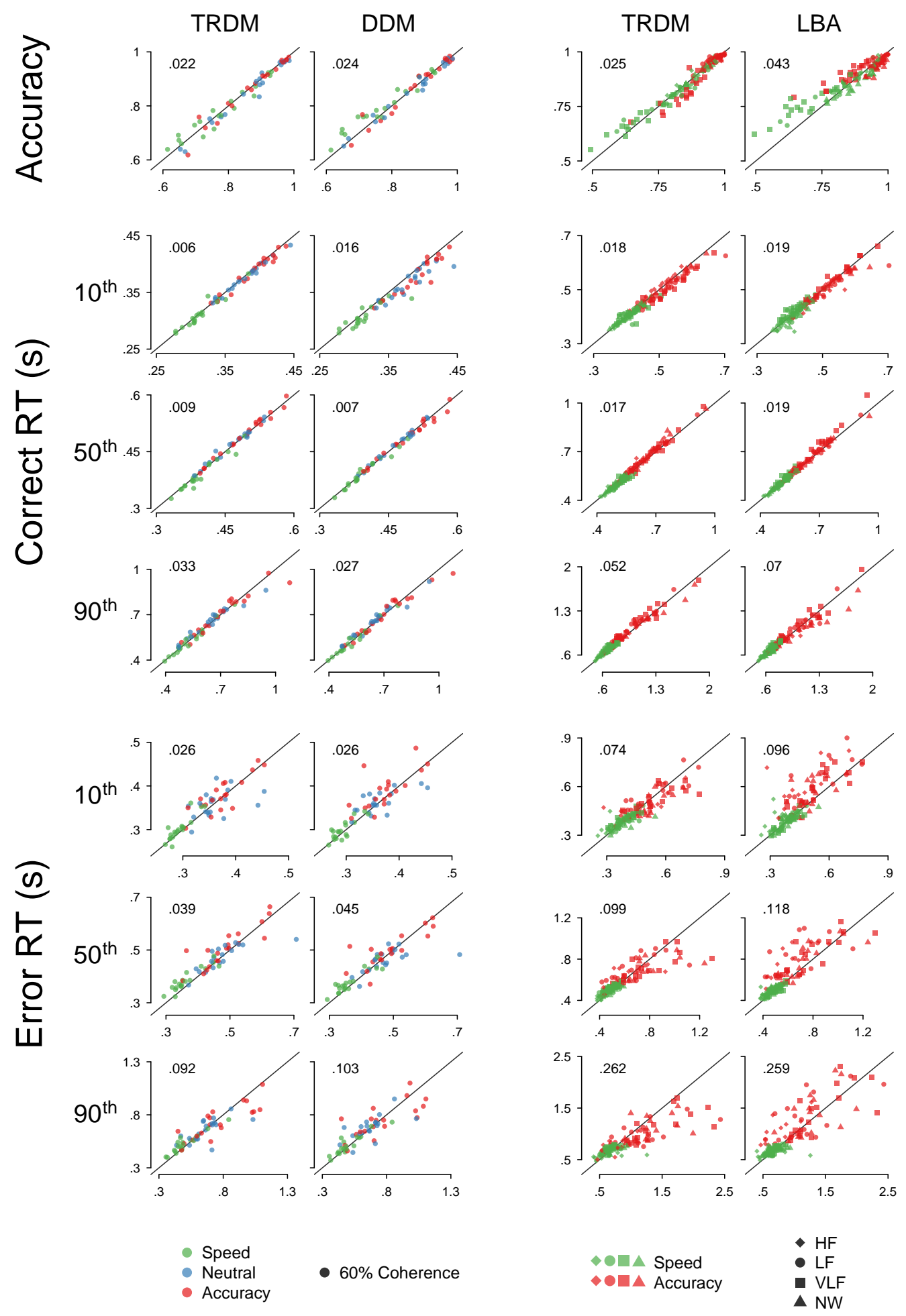

Figure 3. Observed ( $x$-axes) vs. mean posterior predictive ( $y$-axes) data for individual participants. The leftmost two columns represent perceptual decisions (Forstmann et al., 2008) and the rightmost two columns represent lexical decisions (Wagenmakers et al., 2008). The TRDM is shown in the $1^{\text {st }}$ and $3^{\text {rd }}$ columns, the DDM in the $2^{\text {nd }}$ column, and the LBA in the $4^{\text {th }}$ column. The upper row shows accuracy, the next three rows show the $10^{t h}, 50^{\text {th }}$ and $90^{t h}$ percentiles of the correct RT distribution, and the final three rows show the $10^{\text {th }}, 50^{\text {th }}$ and $90^{\text {th }}$ percentiles of the error RT distribution. Each dot represents a single participant in a single experimental condition. Symbol color represents instruction emphasis (speed, neutral, accuracy) and symbol shape represents stimulus condition (perceptual decisions: coherence; lexical decisions: word frequency). The root mean square error is shown in the 
level mean $(\mu)$ parameter estimates for the best TRDM models. We also show in Tables 3 and 4 that the TRDM has good parameter recovery properties for both data sets. Appendix $\mathrm{D}$ details our approach to parameter recovery. In the tables we report the recovery results in terms of the difference between the parameters estimated from simulated data and the parameters that were used to generate those simulated data. Perfect recovery is centered at 0 , with good coverage indicated when the $95 \%$ CI contains the data-generating values (i.e., when it contains 0 ). The difference distribution was close to 0 relative to the degree of uncertainty in estimation for all TRDM parameters, which indicates good parameter recovery, ensuring it is safe to interpret the parameter estimates.

The perceptual-decision parameter estimates in Table 3 display all of the hallmarks of previous speed-accuracy tradeoff explanations: when the accuracy of decisions is emphasized evidence thresholds $\left(\alpha_{E, \mu}\right)$ and non-decision times $\left(\tau_{E}\right)$ are higher (e.g., Ratcliff \& Rouder, 1998; Ratcliff et al., 2016) and mean drift rates $\left(\gamma_{E, \mu}\right)$ are lower (e.g., Rae et al., 2014). There is also a large effect on the timer, almost doubling its rate $\left(\gamma_{T}\right)$. In order to compare the different mechanisms we calculated standardized effect size $(z)$ estimates based on the distribution of the average over participants of differences in speed and accuracy parameters for each posterior sampling iteration (Strickland et al., 2018, details in Appendix E). Timer rate and non-decision time effects were the largest $\left(z\left(\gamma_{T}\right)=13.1\right.$ and $\left.z\left(\tau_{E}\right)=14.1\right)$ with the mean evidence rate intermediate $\left(z\left(\gamma_{E, \mu}\right)=5.9\right)$ and threshold effect the smallest $\left(z\left(\alpha_{E, \mu}\right)=\right.$ $3.5)$, consistent with the model selection results. Appendix E also provides standardized effect estimates for the best performing versions of the competitor models. The LBA also had substantial threshold and rate mean and sensitivity effects $\left(z^{\prime} s>13\right)$ and the DDM substantial threshold and sensitivity effects $\left(z^{\prime} s>21\right)$ in the expected directions. The RDM, which is identical to the TRDM except that it lacks a timer, had substantial effects for threshold, non-decision time and mean rate $\left(z^{\prime} s>8\right)$ in the same direction as those for the TRDM. It also has greater sensitivity in the accuracy condition $\left(z\left(\gamma_{E, \zeta}\right)=8.6\right)$, which has the same effect as a decrease in timer rate in the TRDM, increasing accuracy, suggesting this is a key effect of the timer that substituted for an effect of time pressure on evidence sensitivity.

Table 3 shows that the TRDM's other parameter estimates are sensible. On average, evidence thresholds are unbiased $\left(\alpha_{E, \zeta}\right)$, and non-decision times $\left(\tau_{E}\right)$ have plausible values that are much larger than the onset time for the timing accumulator, consistent with the former time having a substantial response-production component. There was also a large difference in the variability of the different diffusion processes: the standard deviation for the timing process is less than half that of the evidence process. This suggests that the act of timing an interval is more precise than extracting information from a stimulus.

Table 4 shows parameter estimates for the lexical-decision data. In this case the largest effect is on the timer rate $\left(z\left(\gamma_{T}\right)=32.4\right)$ followed by smaller but still substantial effects on mean rates $\left(z\left(\gamma_{E, \mu}\right)=17.3\right)$ and non-decision times $\left(z\left(\tau_{E}\right)=14.8\right)$, and a smaller effect on thresholds $\left(z\left(\alpha_{E, \mu}\right)=4.1\right)$, all in the expected directions. Although effects on mean sensitivity for low and very low frequency words were negligible $\left(z^{\prime} s<.9\right)$, in contrast to the perceptual data there was a substantial effect on evidence sensitivity for non-words $\left(z\left(\gamma_{E, \zeta}\right)=12.7\right)$ and smaller effect for high frequency words $\left(z\left(\gamma_{E, \zeta}\right)=4.1\right)$. These latter effects were in the opposite direction to those conventionally seen in the LBA and RDM (effects which we also found here, see Appendix E) with an increase in sensitivity under 
Table 3

TRDM parameter estimates and parameter recovery for perceptual decisions (Forstmann et al., 2008). Estimates and recovered values are shown as a function of the instruction emphasis (speed, neutral, accuracy) where they were free to vary over this factor in the DIC-best model (cf. Table 1). Column 4 shows the posterior median of the population mean distribution transformed to the natural scale, with underset values showing $95 \%$ credible interval in parentheses. Column 5 shows parameter recovery in terms of summary statistics of the difference distribution between the median of the participant-level posterior distributions estimated from the simulated data and the data-generating parameters. Cell entries show the median and underset values show the 95\% credible interval of the difference distribution. Positive values indicate over-estimation in parameter recovery and negative values indicate under-estimation in parameter recovery, relative to the data-generating values.

\begin{tabular}{|c|c|c|c|c|}
\hline Process & Parameter & Emphasis & $\begin{array}{c}\text { Posterior Median } \\
(95 \% \mathrm{CI})\end{array}$ & $\begin{array}{c}\text { Parameter Recovery } \\
(95 \% \mathrm{CI})\end{array}$ \\
\hline \multirow{12}{*}{ Evidence } & \multirow{3}{*}{$\begin{array}{l}\text { Rate } \\
\left(\gamma_{E, \mu}\right)\end{array}$} & Speed & $\begin{array}{c}2.23 \\
(1.87,2.65)\end{array}$ & $\begin{array}{c}.07 \\
(-.62, .83)\end{array}$ \\
\hline & & Neutral & $\begin{array}{c}1.89 \\
(1.68,2.12)\end{array}$ & $\begin{array}{c}-.14 \\
(-.75, .44)\end{array}$ \\
\hline & & Accuracy & $\begin{array}{c}1.59 \\
(1.27,1.99)\end{array}$ & $\begin{array}{c}-.16 \\
(-1.06, .62)\end{array}$ \\
\hline & $\begin{array}{c}\text { Sensitivity } \\
\left(\gamma_{E, \zeta}\right)\end{array}$ & - & $\begin{array}{c}3.99 \\
(2.71,4.98) \\
\end{array}$ & $\begin{array}{c}-.24 \\
(-2.34, .83)\end{array}$ \\
\hline & \multirow{3}{*}{$\underset{\left(\alpha_{E, \mu}\right)}{\text { Threshold }}$} & Speed & 1 & - \\
\hline & & Neutral & $\begin{array}{c}1.05 \\
(.98,1.11)\end{array}$ & $\begin{array}{c}-.05 \\
(-.27, .13)\end{array}$ \\
\hline & & Accuracy & $\begin{array}{c}1.08 \\
(.98,1.19)\end{array}$ & $\begin{array}{c}0.07 \\
(-.36, .22) \\
\end{array}$ \\
\hline & $\begin{array}{c}\text { Bias } \\
\left(\alpha_{E, \zeta}\right) \\
\end{array}$ & - & $\begin{array}{c}-.11 \\
(-.18,-.04) \\
\end{array}$ & $\begin{array}{c}0 \\
(-.07, .10) \\
\end{array}$ \\
\hline & $\begin{array}{c}\text { Variability } \\
\left(\sigma_{E}\right)\end{array}$ & - & $\begin{array}{c}1.33 \\
(1.21,1.45) \\
\end{array}$ & $\begin{array}{c}-.07 \\
(-.55, .22)\end{array}$ \\
\hline & \multirow{3}{*}{$\underset{\left(\tau_{E}\right)}{\text { Non-decision time }}$} & Speed & $\begin{array}{c}.24 \\
(.23, .25)\end{array}$ & $\begin{array}{c}0 \\
(-.05, .02)\end{array}$ \\
\hline & & Neutral & $(.27, .30)$ & $\begin{array}{c}0 \\
(-.04, .02)\end{array}$ \\
\hline & & Accuracy & $(.28, .31)$ & $\begin{array}{c}0 \\
(-.04, .03)\end{array}$ \\
\hline \multirow{6}{*}{ Timing } & \multirow{3}{*}{$\underset{\left(\gamma_{T}\right)}{\operatorname{Rate}}$} & Speed & $\begin{array}{c}1.66 \\
(1.29,2.13)\end{array}$ & $\begin{array}{c}.03 \\
(-.46, .53)\end{array}$ \\
\hline & & Neutral & $\begin{array}{c}1.12 \\
(.91,1.38)\end{array}$ & $\begin{array}{c}.02 \\
(-.30, .33)\end{array}$ \\
\hline & & Accuracy & $\begin{array}{c}.86 \\
(.67,1.11) \\
\end{array}$ & $\begin{array}{c}.01 \\
(-.34, .43)\end{array}$ \\
\hline & $\begin{array}{c}\text { Threshold } \\
\left(\alpha_{T}\right)\end{array}$ & - & 1 & - \\
\hline & $\begin{array}{c}\text { Variability } \\
\left(\sigma_{T}\right)\end{array}$ & - & $\begin{array}{l}.51 \\
(.45, .57) \\
\end{array}$ & $\begin{array}{c}-.01 \\
(-.09, .06) \\
\end{array}$ \\
\hline & $\underset{\left(\tau_{T}\right)}{\text { Onset }}$ & - & .05 & - \\
\hline
\end{tabular}


Table 4

TRDM parameter estimates for lexical decisions (Wagenmakers et al., 2008). Estimates are shown as a function of the instruction emphasis (speed, accuracy) and lexical class (HF, LF, $V L F, N W$ ) where they were free to vary over these factors in the DIC-best model (cf. Table 2). Column 5 shows the posterior median of the population mean distribution transformed to the natural scale, with underset values showing $95 \%$ credible interval in parentheses. Column 6 shows parameter recovery in the same format as Table 3.

\begin{tabular}{|c|c|c|c|c|c|}
\hline Process & Parameter & Emphasis & Stimulus & $\begin{array}{c}\text { Posterior Median } \\
\quad(95 \% \mathrm{CI})\end{array}$ & $\begin{array}{c}\text { Parameter Recovery } \\
(95 \% \mathrm{CI})\end{array}$ \\
\hline \multirow{16}{*}{ Evidence } & \multirow{2}{*}{$\begin{array}{l}\text { Rate } \\
\left(\gamma_{E, \mu}\right)\end{array}$} & Speed & - & $\begin{array}{c}1.86 \\
(1.61,2.15)\end{array}$ & $\begin{array}{c}-.01 \\
(-.22, .20)\end{array}$ \\
\hline & & Accuracy & - & $\begin{array}{c}1.14 \\
(.94,1.38)\end{array}$ & $\begin{array}{c}.03 \\
(-.31, .27)\end{array}$ \\
\hline & \multirow{8}{*}{$\begin{array}{l}\text { Sensitivity } \\
\left(\gamma_{E, \zeta}\right)\end{array}$} & \multirow{4}{*}{ Speed } & $\mathrm{HF}$ & $\begin{array}{c}5.91 \\
(5.12,6.49)\end{array}$ & $\begin{array}{c}-.21 \\
(-1.32, .88)\end{array}$ \\
\hline & & & $\mathrm{LF}$ & $\begin{array}{c}3.16 \\
(2.69,3.58)\end{array}$ & $\begin{array}{c}-.17 \\
(-.80, .59)\end{array}$ \\
\hline & & & VLF & $\begin{array}{c}1.82 \\
(1.41,2.21)\end{array}$ & $\begin{array}{c}-.01 \\
(-.65, .86)\end{array}$ \\
\hline & & & NW & $\begin{array}{c}4.01 \\
(3.55,4.41)\end{array}$ & $\begin{array}{c}.10 \\
(-.89, .67)\end{array}$ \\
\hline & & \multirow{4}{*}{ Accuracy } & HF & $\begin{array}{c}4.85 \\
(3.71,5.65)\end{array}$ & $\begin{array}{c}-.12 \\
(-1.58, .91)\end{array}$ \\
\hline & & & $\mathrm{LF}$ & $\begin{array}{c}3.06 \\
(2.57,3.52)\end{array}$ & $\begin{array}{c}-.08 \\
(-.89, .58)\end{array}$ \\
\hline & & & VLF & $\begin{array}{c}1.91 \\
(1.59,2.22)\end{array}$ & $\begin{array}{c}-.08 \\
(-.78, .35)\end{array}$ \\
\hline & & & NW & $\begin{array}{c}2.99 \\
(2.55,3.37)\end{array}$ & $\begin{array}{c}-.01 \\
(-.69, .53)\end{array}$ \\
\hline & \multirow{2}{*}{$\underset{\left(\alpha_{E, \mu}\right)}{\text { Threshold }}$} & Speed & - & 1 & - \\
\hline & & Accuracy & - & $\begin{array}{c}1.07 \\
(.98,1.17)\end{array}$ & $\begin{array}{c}0 \\
(-.19, .21)\end{array}$ \\
\hline & $\begin{array}{c}\text { Bias } \\
\left(\alpha_{E, \zeta}\right)\end{array}$ & - & - & $\begin{array}{c}.04 \\
(-.02, .11)\end{array}$ & $\begin{array}{c}.01 \\
(-.06, .09)\end{array}$ \\
\hline & $\begin{array}{c}\text { Variability } \\
\left(\sigma_{E}\right)\end{array}$ & - & - & $\begin{array}{c}1.10 \\
(1.03,1.18) \\
\end{array}$ & $\begin{array}{c}0 \\
(-.19, .13) \\
\end{array}$ \\
\hline & \multirow{2}{*}{$\underset{\left(\tau_{E}\right)}{\text { Non-decision time }}$} & Speed & - & $\begin{array}{c}.31 \\
(.29, .33)\end{array}$ & $\begin{array}{c}0 \\
(-.02, .02)\end{array}$ \\
\hline & & Accuracy & - & $\begin{array}{c}.36 \\
(.33, .38)\end{array}$ & $\begin{array}{c}0 \\
(-.03, .02)\end{array}$ \\
\hline \multirow{5}{*}{ Timing } & \multirow{2}{*}{$\underset{\left(\gamma_{T}\right)}{\operatorname{Rate}}$} & Speed & - & $\begin{array}{c}1.12 \\
(.98,1.29)\end{array}$ & $\begin{array}{c}0 \\
(-.28, .22)\end{array}$ \\
\hline & & Accuracy & - & $\begin{array}{c}.23 \\
(.12, .46)\end{array}$ & $\begin{array}{c}0 \\
(-.25, .16)\end{array}$ \\
\hline & $\begin{array}{c}\text { Threshold } \\
\left(\alpha_{T}\right)\end{array}$ & - & - & 1 & - \\
\hline & $\begin{array}{c}\text { Variability } \\
\left(\sigma_{T}\right)\end{array}$ & - & - & $\begin{array}{c}.53 \\
(.49, .57)\end{array}$ & $\begin{array}{c}0 \\
(-.05, .05)\end{array}$ \\
\hline & $\underset{\left(\tau_{T}\right)}{\text { Onset }}$ & - & - & .05 & - \\
\hline
\end{tabular}


time pressure. We discuss the psychological implications of these findings further below, but first we examine how the TRDM accounts for the relative speed of correct vs. error responses.

The Latent Dynamics of Information-Controlled Decisions. Finally, we examine how the TRDM's novel timer mechanism addresses the fine detail of time pressure effects related to the relative speed of error and correct responses. The findings that errors tend to be slower than correct responses when accuracy is emphasized, and as fast or faster when speed is emphasized, have been important benchmarks for assessing the adequacy of evidence accumulation models that necessitated the inclusion of trial-to-trial parameter variability (Ratcliff \& Rouder, 1998). We now show that these effects can instead be explained by changes in how often the timer wins as a function of response speed.

In particular, we used individual-participant parameter estimates to examine how the posterior predictive proportion of trials terminated by the timing process varied with time pressure. We found that the increase in clock speed under time pressure was sufficient to overcome the increased speed of evidence accumulation. For perceptual decisions, when instructed to emphasize decision speed approximately $27 \%$ (95\% CI $[24,31])$ of decisions were a result of the timing process crossing threshold before either of the accumulators in the evidence process, which decreased to $20 \%[17,22]$ and $15 \%[12,17]$ for the neutral and accuracy emphasis conditions, respectively. These differences between conditions were reliable: the speed condition had an average of $7 \%[3,12]$ and $12 \%[9,16]$ more timer responses than the neutral and accuracy conditions, respectively, and the neutral condition had $5 \%$ $[2,8]$ more timer responses than the accuracy condition. Similarly for lexical decisions, when task instructions emphasized response speed $21 \%[20,22]$ of decisions were predicted to be the outcome of the timing process, decreasing to $7 \%[6,8]$ for accuracy emphasis instructions, which was also a reliable difference between conditions $(14 \%[13,16])$.

Figure 4 provides conditional accuracy functions (CAFs; i.e., accuracy as a function of RT, see Ridderinkhof, 2002) separately for each task (columns) and time-pressure condition (rows). It shows that the TRDM captures the conventional pattern of a greater preponderance of fast errors under speed emphasis. In order to examine how the TRDM achieves this, we also plot the proportion of responses due to the timer as a function of RT. That is, for each task we calculated the probability of the correct, error and timer accumulators crossing threshold first for every observed RT. Figure 4 plots our findings separated by the speed-accuracy tradeoff manipulation and aggregated over stimulus conditions and participants. In speeded conditions the timer mainly produced what can be characterized as "fast guesses", rapid responses that are responsible for the sharp initial rise of the CAF. In accuracy conditions the proportion of timer responses was both lower and varied less as a function of RT, with only a small rise at short RTs, consistent with the much flatter observed CAFs and slower error than correct responses. In contrast, the probability of evidence-based errors changed little with RT, indicating that the timer mechanism is mainly responsible for differences in the speed of error and correct responses.

\section{Discussion}

The TRDM provides a better quantitative explanation of the trends in speed-accuracy tradeoff data in both the perceptual and lexical decision data sets than the DDM, RDM and LBA. This is a notable result given that the weight of evidence in the literature suggests 
Perceptual Decisions

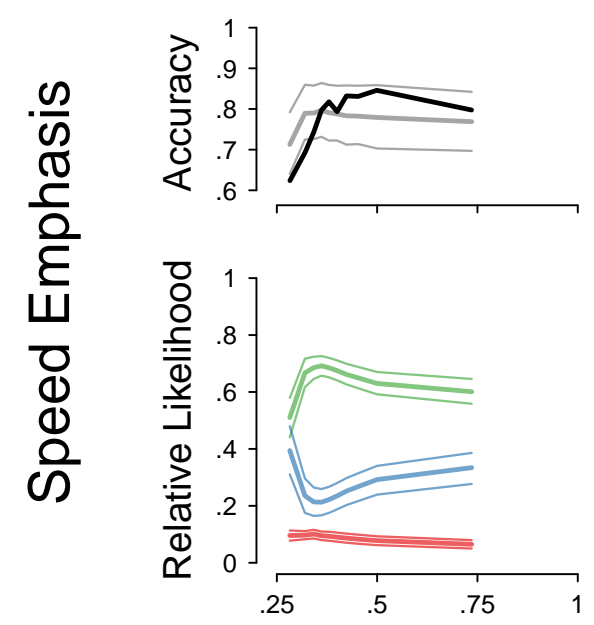

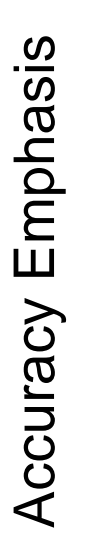
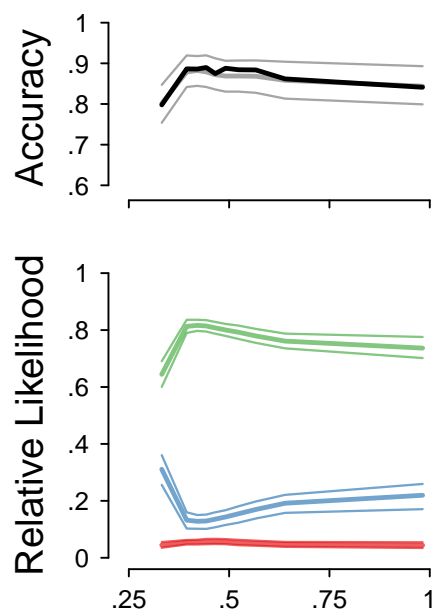

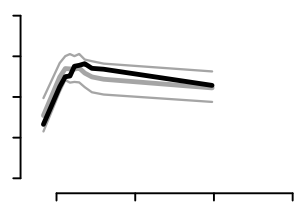

\section{Lexical \\ Decisions}
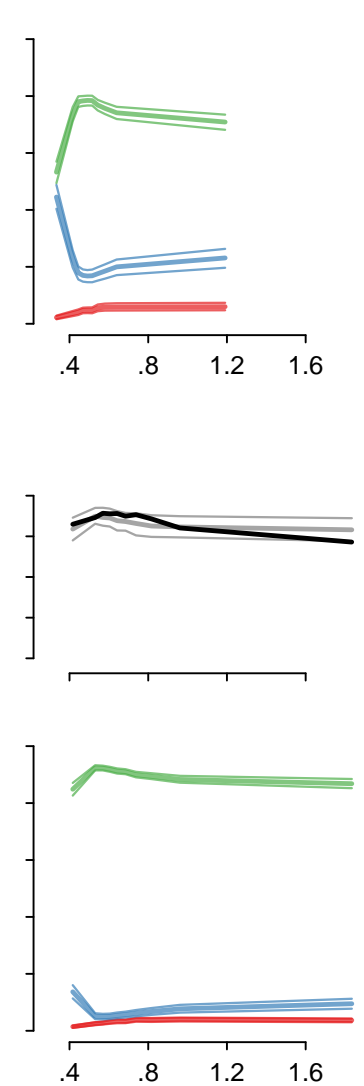

Response Time (s)

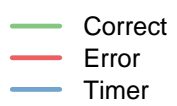

Data
— Model

Figure 4. Posterior predicted probability of each accumulator winning the TRDM race (crossing threshold first; colored panels) and the conditional accuracy function (gray scale panels) as a function of the range of observed RTs in each experiment ( $x$-axes). Perceptual decisions (Forstmann et al., 2008) and lexical decisions (Wagenmakers et al., 2008) are shown in columns. Speed and accuracy emphasis conditions are shown in rows; for visualization purposes we aggregated over the neutral and accuracy emphasis conditions for the perceptual decisions. Colors represent accumulators: correct in green, error in red, and timer in blue. Observed and posterior predicted accuracy are shown in black and gray, respectively. The mean and standard error (.16 and .84 quantiles) of the posterior predictive distribution for individual trials, averaged across participants, are shown in heavy and thin lines, respectively. 
that choice-response time models require some form of across-trial variability in parameters to account for the relative speed of error and correct responses under different instruction regimes, yet the TRDM does not include any such sources of variability. Under speed emphasis the tendency for errors to be faster than correct responses is captured by an increase in cases where fast responses are due to the timer. Under accuracy emphasis the tendency for errors to be slower than correct responses is captured by the evidence process.

The TRDM provides a new theoretical perspective on decisions under time pressure. When decision speed is emphasized, the conventional explanation is that people strategically adjust their level of response caution, accumulating less evidence prior to decision commitment. This is an indirect way for the decision maker to adapt to changing task demands: adjusting the level of evidence in response to an instruction about time - the instruction is commonly to "be speedy" and not "collect less evidence". The TRDM allows for adaptation to time pressure in the conventional manner, by accumulating less evidence, but it also allows the decision maker to directly adjust the amount of time they are willing to wait before they 'time out' their decision, by setting timing drift rates to be faster under speed emphasis than accuracy emphasis conditions.

Although the TRDM suggests an important role for the timing process it also provides a nuanced interpretation of the speed-accuracy tradeoff reflecting differing roles for several elements of conventional accounts. When instructed to emphasize decision speed, people appear to be able to make a number of adaptations to their decision style: they increase the mean rate of evidence accumulation and have faster non-decision times as well as setting a shorter internal time limit on the decision. Surprisingly, in contrast to the classic assumption that threshold effects are the primary mechanism mediating time-pressure effects, they have at best a secondary influence according to the TRDM, at least in the data considered here. Perhaps also surprising, for lexical decisions there was some support for increased sensitivity with time pressure, at least for non-words and high-frequency words. This pattern of drift rate effects - an increase in both their mean and to a lesser degree their sensitivity contrasts with the LBA and RDM where the mean also increases but there is also a strong decrease in sensitivity. The latter decrease partially mediates the ability of these models to explain a decrease in accuracy with time pressure, whereas for the TRDM it is explained by an increase in inaccurate timer-based responses.

Given the fact that an increase in sensitivity was not supported in the TRDM's account of perceptual decisions, and even for lexical decisions a model with no sensitivity effect was still superior to the best competitor (LBA) model in terms of DIC, further investigation is needed to substantiate this aspect of our findings. In contrast, we found clear and consistent evidence that in both perceptual and lexical decisions participants responded to time pressure by decreasing non-decision time, increasing the mean evidence rate and to an even greater degree increasing their timer's rate so that it more often beat evidence accumulation than when accuracy was emphasized.

\section{Time-Controlled Choice: Explicit and Implicit Deadlines}

In a similar vein to our findings about decisions made under time pressure, we argue that the most natural adaptation to decisions made in the presence of response deadlines is to calibrate the expected finishing time of an internal timer to help ensure that the deadline is met. To date, cognitive models that have most effectively explained decisions 
with deadlines assume that evidence thresholds are dynamically adjusted as a function of elapsed decision time (e.g., Cisek et al., 2009; Evans, Hawkins, \& Brown, 2019; Frazier \& Yu, 2008; Hawkins, Forstmann, et al., 2015; Hawkins, Wagenmakers, Ratcliff, \& Brown, 2015; Katsimpokis, Hawkins, \& Van Maanen, in press; Palestro, Weichart, Sederberg, \& Turner, 2018). That is, they posit an extra mechanism (dynamic threshold adjustment) that is essentially a pseudo timer; thresholds can only collapse as a function of decision time if there is a mechanism that monitors time, which (implicitly) informs when the thresholds should be adjusted. This does not build on what we know about timing, where formal theories of interval reproduction are the norm (for reviews, see Grondin, 2010; Simen, Rivest, Ludvig, Balci, \& Killeen, 2013). Nevertheless, there are similarities between collapsing threshold models and the TRDM's timing process such that one might view the timer as a pseudo collapse; that is, plotting $1-F_{T}(t)$ (Equation 1) produces a collapsing threshold-like shape (cf. Hawkins, Forstmann, et al., 2015; Palestro et al., 2018). However, the two are not formally equivalent. The architecture of collapsing threshold models corresponds to an assumption of 'partial information' in the TRDM. Under the settings we consider here, the predicted response of the timing process is not structurally linked to the state of the evidence accumulation process. To more closely mirror the structure of a collapsing threshold model, the TRDM would need to make a partial information assumption - guessing proportional to the current 'winner' of the evidence process. As noted in Part 2 of the paper, we do not study partial information in the TRDM as this more complex assumption was not required in any data set we examined. However, future research may further consider this assumption.

We argue that the TRDM has the desirable feature that it unifies models from the decision and timing literatures, integrating what we know about decisions with what we know about time estimation. It can also serve as a 'drop-in replacement' for collapsing thresholds models with the advantage of an analytic likelihood function. Here, we test the hypothesis that the TRDM provides a unified account of responding to deadlines against two types of deadline data: explicit deadlines - where there is an experimenter-defined upper limit on the available decision time - and implicit deadlines - where the task environment incentivises (or disincentivises) responses at particular times.

Data. We applied the TRDM to two data sets that have been previously shown to require dynamic decision models. We provide a brief overview of the data sets here; for full details we refer the reader to the primary sources.

Frazier and $\mathrm{Yu}$ (2008) proved that when there is an upper bound on decision time - most commonly implemented as an experimenter-imposed response deadline - dynamic decision making models with collapsing thresholds optimize task performance in the sense that they maximize long-run reward. Experiment 2 of Evans, Hawkins, and Brown (2019) tested this hypothesis. Forty-one participants made decisions about random dot motion across a range of difficulty levels: $0 \%, 5 \%, 10 \%$ and 40\%, randomized across trials. All trials had a response deadline of $1.3 \mathrm{~s}$. If the participant did not respond before the explicit deadline the stimulus was removed from screen, a response was no longer accepted for the trial, and the participant received a time-out for missing the deadline before proceeding to the next trial. Evans, Hawkins, and Brown (2019) found greater support across participants for a DDM with collapsing thresholds than a DDM with fixed thresholds.

In a reevaluation of many data sets, Hawkins, Forstmann, et al. (2015) found the 
strongest evidence for collapsing thresholds for two monkeys of Roitman and Shadlen (2002); see also Ditterich (2006); Hawkins, Wagenmakers, et al. (2015). Recently, Evans and Hawkins (2019) verified in humans that the strong preference for collapsing thresholds in Roitman and Shadlen's (2002) monkeys can likely be attributed to a timing procedure in their experimental protocol that aimed to prevent the monkeys from responding too rapidly: the reward for a correct response was withheld until a minimum time after the stimulus onset, no matter how quickly the monkey made a response. This encouraged the monkeys to withhold their responses until the reward delay had passed and then produce the bulk of their responses in a narrow window. We consider Roitman and Shadlen's (2002) task design to have an implicit deadline: the monkeys aimed to respond after the time the reward became available, unlike the humans that aimed to respond before the explicit deadline in Evans, Hawkins, and Brown (2019). As a consequence of responding after the reward delay, the distribution of RTs was much more symmetric than is typically observed; it is this unusual and almost Gaussian outcome that the DDM with fixed thresholds cannot explain. Hence, here we treat this experiment as having an implicit deadline incentivised by the experimental protocol that is modeled by the TRDM's timing accumulator.

Roitman and Shadlen's (2002) monkeys made decisions about random dot motion with choices indicated by eye movements. Decision difficulty was randomly varied from one decision to the next across six levels: $0 \%, 3.2 \%, 6.4 \%, 12.8 \%, 25.6 \%$, and $51.2 \%$ coherence. Roitman and Shadlen (2002) had one monkey complete 2614 trials (monkey B) and another completed 3534 trials (monkey N). The reward delay for monkey B was $800 \mathrm{~ms}$ and for monkey $\mathrm{N}$ it was $1200 \mathrm{~ms}$.

Goodness of Fit. We compare the TRDM to the set of three competitor models introduced in the previous section, which includes the DDM with fixed thresholds. For all four models and both data sets, we estimated a separate evidence sensitivity parameter for each motion coherence condition $\left(\gamma_{E, \zeta}, \gamma_{\zeta}\right.$ or $v$, depending on the model). All other parameters were estimated to a single value across conditions, including the mean evidence rate for the TRDM, RDM and LBA models. Appendix B provides complete parameterization details.

We do not formally compare the TRDM to the DDM with collapsing thresholds. Collapsing thresholds can be difficult to reliably estimate from data in a way that supports model selection criteria that account for a model's goodness of fit as well as its parametric and functional form complexity (Evans, Trueblood, \& Holmes, 2019). This is because the models require simulation-based estimation that sometimes bring additional complexities (e.g., integral equation methods or probability density approximation; Smith, 2000; Turner \& Sederberg, 2014), although these difficulties have been surmounted in some cases (e.g., Evans, Hawkins, \& Brown, 2019; Turner, Sederberg, \& McClelland, 2016; Voskuilen, Ratcliff, \& Smith, 2016). We restrict our focus to likelihood-based models so that model selection criteria can be estimated on a common-ground metric. Nevertheless, for visual comparison we take the predictions of collapsing thresholds models as they were reported in previous publications (Evans, Hawkins, \& Brown, 2019 for explicit deadlines, Hawkins, Forstmann, et al., 2015 for implicit deadlines) and present them alongside the TRDM and DDM with fixed thresholds. This highlights the capacity of the TRDM to explain similar trends in data as the collapsing thresholds model.

The DICs in Table 5 show that the TRDM provided by far the best explanation of the 
models without collapsing thresholds. Figure 5 shows observed vs. posterior predicted data for individual participants for the TRDM, DDM with fixed thresholds (estimated here), and DDM with collapsing thresholds (estimated in previous publications). To facilitate comparison with a collapsing thresholds DDM, Figure 5 displays predictions of the DDM with fixed thresholds rather than the second best performing model, which was the RDM in both cases.

Table 5

DIC model comparison values for time-controlled decisions. Rows show the data sets and models. Column 3 shows the number of model parameters freely estimated for each participant. Column 4 shows DICs zero-referenced to the DIC-preferred model such that positive values indicate a poorer explanation of the data. Rows are ordered by DIC separately for each data set.

\begin{tabular}{cccc} 
Data Set & Model & $\begin{array}{c}\text { Number of } \\
\text { Free Parameters }\end{array}$ & $\Delta$ DIC \\
\hline \multirow{5}{*}{ Explicit Deadlines } & TRDM & 10 & 0 \\
& RDM & 8 & 649 \\
& DDM & 10 & 813 \\
& LBA & 10 & 1,246 \\
\hline \multirow{5}{*}{ Implicit Deadlines } & TRDM & 12 & 0 \\
& RDM & 10 & 512 \\
& LBA & 12 & 791 \\
& DDM & 12 & 1,208
\end{tabular}

All three models provided a good account of individual participant accuracy data. For the explicit deadlines, the DDM with fixed thresholds tended to underpredict the fastest correct responses $\left(10^{\text {th }}\right.$ percentile). It also underpredicted the median correct RTs in the more difficult coherence levels, and overpredicted the frequency with which participants missed the deadline. For the implicit deadlines the DDM misfit was more marked, predicting too much variance (underpredicting the fastest responses, overpredicting the slowest responses) as noted in previous analyses of this data set (Ditterich, 2006; Hawkins, Wagenmakers, et al., 2015). In contrast, there were no signs of systematic misfit for the TRDM in either data set. There was not a great deal of difference in fit between the TRDM and the DDM with collapsing thresholds; RMSE was systematically slightly better for the TRDM with explicit deadlines, whereas overall RMSE was similar for implicit deadlines. Because accuracy was very high for the easiest decisions (for the highest coherence stimuli), the error quantiles in this cell are predicted with considerable noise for all models owing to there being so few data points.

Figure 6 provides data and model predictions aggregated across participants. It shows some of the key features in data in a more pronounced manner, particularly the reduced positive skew of the RT distribution that is not captured by the DDM with fixed thresholds, since the model is constrained to predict a strictly positively skewed distribution (Ratcliff, 2002). Instead, the DDM predicts too much variance relative to data; the leading edge is faster and the right tail slower than in data, or both. This also means the DDM misses the relative speed of correct and error responses, in particular for the implicit deadlines. 

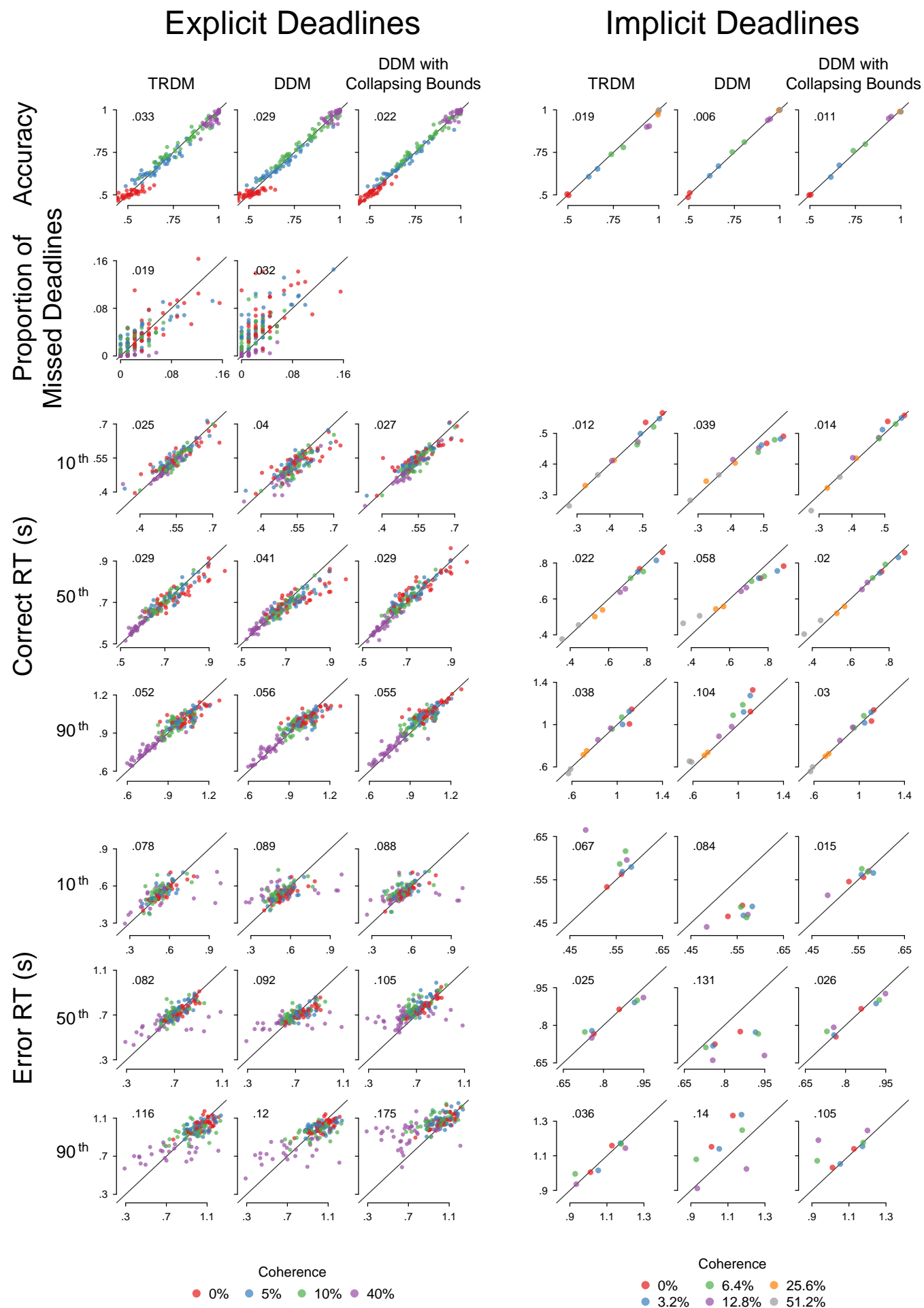

$0 \% \cdot 5 \% \cdot 10 \% \cdot 40 \%$

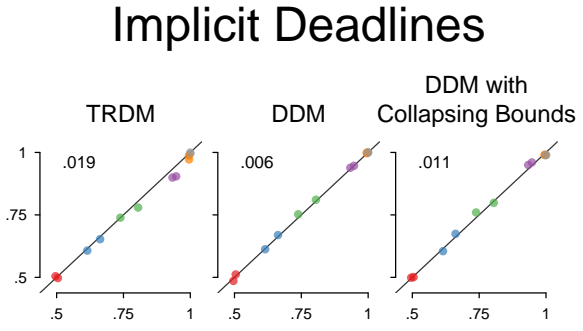




\section{Explicit Deadlines Implicit Deadlines}

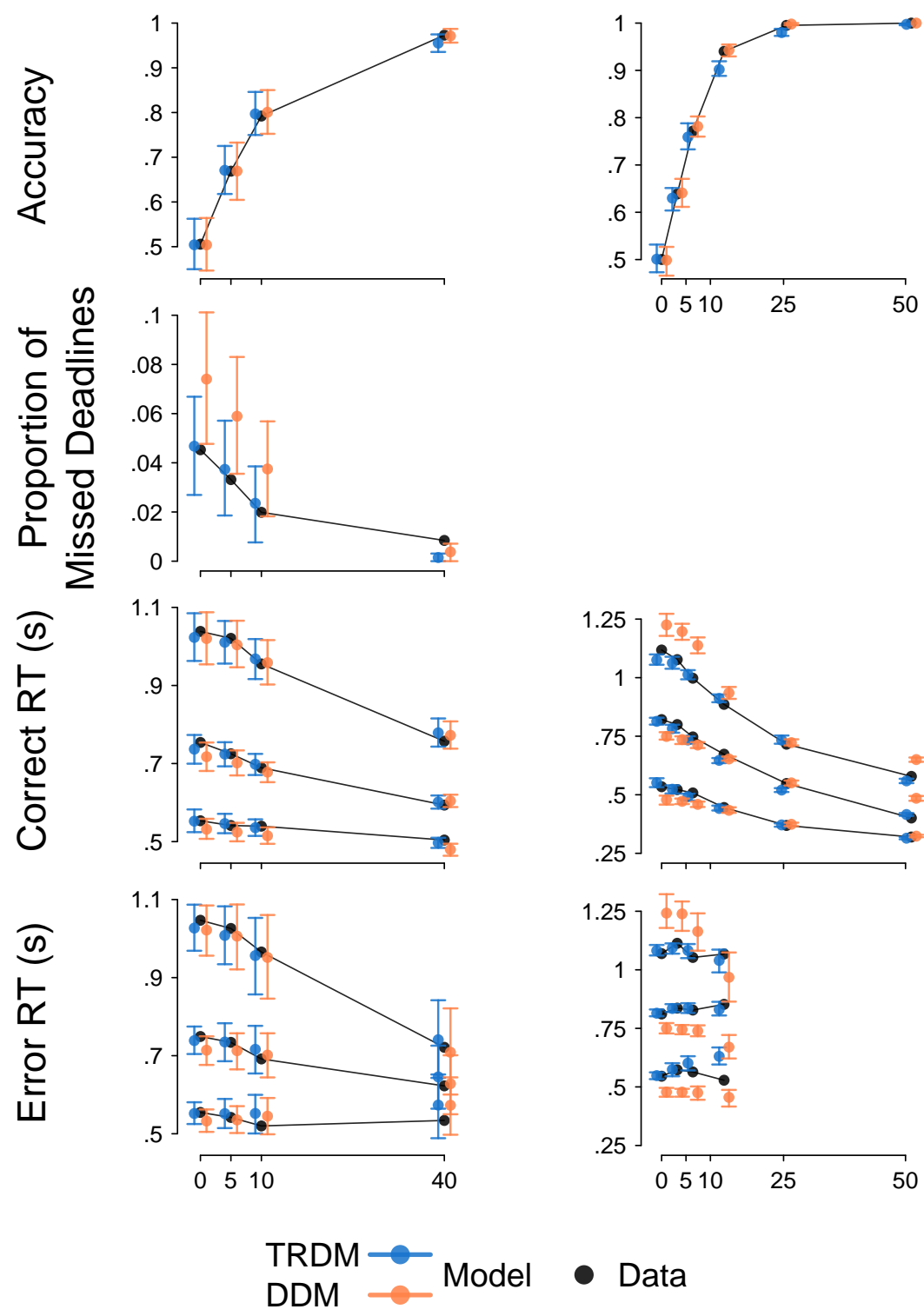

Figure 6. Observed and posterior predictive distribution (TRDM in blue, DDM with fixed thresholds in orange) for decisions with explicit deadlines (left; Evans, Hawkins, \& Brown, 2019) and implicit deadlines (right; Roitman \& Shadlen, 2002). Rows represent different statistics: choice accuracy (upper), proportion of missed deadlines (only defined in the context of explicit deadlines), and correct and error RTs (lower two rows), where vertically placed dots for the latter represent percentiles of the distributions $\left(10^{t h}, 50^{t h}, 90^{t h}\right)$. Uncertainty bars represent the standard error of the posterior predictive distribution. $x$-axes in each panel represent the motion coherence manipulation. 
To truncate the predicted distribution of RTs the DDM requires modification to its core architecture, such as collapsing thresholds or an urgency signal.

The TRDM can naturally predict truncated RT distributions with no changes to the model architecture. The TRDM predicts both the appropriate RT variance and the pattern of error responses that are considerably slower than correct responses. This was even so in the extreme case in Roitman and Shadlen (2002) where the monkeys generated approximately Gaussian distributed RTs. In this account the decision maker calibrates their timing process to terminate the hardest decisions early (with low quality stimulus evidence) so as to avoid spending too much time when there is a low chance of a successful choice outcome, while still allowing the easiest decisions (with high quality stimulus evidence) to terminate with high probability in the evidence accumulation process. This produces behavior consistent with the reward rate maximization argument often used to motivate collapsing threshold models (Drugowitsch et al., 2012).

This interplay between the evidence and timing processes - where the timing process censors the slowest responses of the evidence process - reduces the skew of the predicted RT distribution relative to evidence-only models. The magnitude of the predicted skew reduction is an increasing function of the rate and precision of the timing process, as this decreases the predicted RT variance (cf. Figure 2). When this effect is strong it also implies that the CAF should decline as RT increases, which, as we discuss in more detail below, was observed in the data (cf. Figure 7).

As shown in Figure 5 for individual participants and in Figure 6 in aggregate, the DDM with fixed thresholds missed substantially more of the explicit deadlines compared to both the data and to the TRDM. This is because the DDM has no mechanism to terminate the decision process when the deadline looms near. In the TRDM, in contrast, once the decision maker has internalized the time at which the deadline appears, they can calibrate their timing process to terminate just prior to the deadline if the evidence accumulation process has not already terminated. This strategy ensures that response opportunities are not missed. A similar motivation holds for collapsing thresholds, which assumes that decision boundaries can collapse just prior to the deadline (Frazier \& Yu, 2008). We do not show the goodness of fit of the collapsing thresholds model to the proportion of missed deadlines as the original publication did not fit the model to these data, so model predictions are not available.

Parameter Estimates. Tables 6 and 7 show parameter estimates and recovery for the two deadline data sets. Parameter recovery was again excellent, indicating the parameters can be safely interpreted. All estimates have psychologically sensible interpretations: drift rate sensitivity increases with increasing motion coherence and non-decision time estimates are plausible in light of the species and response modality differences. As in the speed-accuracy tradeoff data sets, we again see that the timing process is less variable than the evidence process.

One difference between the two deadline data sets is that the mean evidence rate was faster than the mean timing rate for the explicit deadlines (1.16 vs. .72) whereas the reverse was true for the implicit deadlines (.83 vs. 1.05). This indicates that the timer had a larger influence on the decision process for the implicit than explicit deadlines, corresponding to a greater predicted proportion of trials terminated by the timing process. 
Table 6

TRDM parameter estimates and parameter recovery for the data set with explicit deadlines (Evans, Hawkins, $\&$ Brown, 2019). Estimates are shown as a function of the stimulus coherence $(0 \%, 5 \%, 10 \%, 40 \%)$ where they were free to vary over this factor in the DICbest model (cf. Table 5). Column 4 shows the posterior median of the population mean distribution transformed to the natural scale, with underset values showing $95 \%$ credible interval in parentheses. Column 5 shows parameter recovery in the same format as Table 3.

\begin{tabular}{|c|c|c|c|c|}
\hline Process & Parameter & Coherence & $\begin{array}{c}\text { Posterior Median } \\
(95 \% \mathrm{CI})\end{array}$ & $\begin{array}{c}\text { Parameter Recovery } \\
(95 \% \text { CI })\end{array}$ \\
\hline \multirow{9}{*}{ Evidence } & $\begin{array}{l}\text { Rate } \\
\left(\gamma_{E, \mu}\right)\end{array}$ & - & $\begin{array}{c}1.16 \\
(1.06,1.28) \\
\end{array}$ & $\begin{array}{c}-.03 \\
(-.44, .18) \\
\end{array}$ \\
\hline & \multirow{4}{*}{$\begin{array}{l}\text { Sensitivity } \\
\quad\left(\gamma_{E, \zeta}\right)\end{array}$} & $0 \%$ & $\begin{array}{c}.03 \\
(-.05, .11)\end{array}$ & $\begin{array}{c}.03 \\
(-.28, .29)\end{array}$ \\
\hline & & $5 \%$ & $\begin{array}{c}.90 \\
(.76,1.04)\end{array}$ & $\begin{array}{l}.05 \\
(-.38, .46)\end{array}$ \\
\hline & & $10 \%$ & $\begin{array}{c}1.83 \\
(1.60,2.06)\end{array}$ & $(-.60, .65)$ \\
\hline & & $40 \%$ & $\begin{array}{c}4.83 \\
(4.37,5.26) \\
\end{array}$ & $\begin{array}{c}.14 \\
(-1.28,1.20)\end{array}$ \\
\hline & $\underset{\left(\alpha_{E, \mu}\right)}{\text { Threshold }}$ & - & 1 & - \\
\hline & $\begin{array}{c}\text { Bias } \\
\left(\alpha_{E, \zeta}\right) \\
\end{array}$ & - & $\begin{array}{c}.07 \\
(.03, .10) \\
\end{array}$ & $\begin{array}{c}0 \\
(-.10, .08) \\
\end{array}$ \\
\hline & $\underset{\left(\sigma_{E}\right)}{\text { Variability }}$ & - & $\begin{array}{c}.86 \\
(.81, .92)\end{array}$ & $\begin{array}{c}.01 \\
(-.14, .15)\end{array}$ \\
\hline & Non-decision time & - & $\begin{array}{c}.36 \\
(.34, .37)\end{array}$ & $\begin{array}{c}0 \\
(-.03, .04)\end{array}$ \\
\hline \multirow{4}{*}{ Timing } & $\underset{\left(\gamma_{T}\right)}{\text { Rate }}$ & - & $\begin{array}{l}.72 \\
(.64, .79)\end{array}$ & $\begin{array}{c}.06 \\
(-.13, .46)\end{array}$ \\
\hline & $\underset{\left(\alpha_{T}\right)}{\text { Threshold }}$ & - & 1 & - \\
\hline & $\begin{array}{c}\text { Variability } \\
\left(\sigma_{T}\right)\end{array}$ & - & $\begin{array}{c}.36 \\
(.31, .43)\end{array}$ & $\begin{array}{c}-.01 \\
(-.15, .08)\end{array}$ \\
\hline & $\underset{\left(\tau_{T}\right)}{\text { Onset }}$ & - & .05 & - \\
\hline
\end{tabular}

Latent Dynamics of Time-Controlled Decisions. We generated the posterior predictive proportion of trials terminated by the timing process to assess how it varied across the levels of the motion coherence difficulty manipulation. For the implicit deadlines, the proportion of timer responses was very large in the most difficult conditions and gradually decreased as decisions became easier; in order from hardest to easiest decisions: $41 \%(95 \%$ CI $[35,46]), 39 \%[33,44], 31 \%[27,36], 17 \%[14,20], 4 \%[2,6]$, and .5\% [.2,1]. There was a similar pattern for the external deadlines data set, though timer responses were generally less frequent: $22 \%[20,24], 21 \%[19,22], 17 \%[16,19], 9 \%[7,10]$. In both data sets the difference between the hardest and easiest conditions was very strong.

Figure 7 shows these results as a function of RT. To simplify visualization we collapsed across some of the coherence conditions in the implicit deadlines data: cells with $50 \%$ mean accuracy are labelled 'no signal', 50-70\% are 'hard', 70-90\% are 'medium', and $>90 \%$ are 'easy'. The relative proportion of evidence vs. timing processes predicted to terminate the decision process shifted systematically across difficulty levels: the timing process 
Table 7

TRDM parameter estimates and parameter recovery for the data set with implicit deadlines (Roitman ES Shadlen, 2002). Estimates are shown as a function of the stimulus coherence $(0 \%, 3.2 \%, 6.4 \%, 12.8 \%, 25.6 \%, 51.2 \%)$ where they were free to vary over this factor in the DIC-best model (cf. Table 5). Column 4 shows the posterior median and 95\% credible interval (underset) of individual participant posterior distributions, then averaged over the participants, transformed to the natural scale. Column 5 shows parameter recovery in the same format as Table 3.

\begin{tabular}{|c|c|c|c|c|}
\hline Process & Parameter & Coherence & $\begin{array}{c}\text { Posterior Median } \\
(95 \% \text { CI })\end{array}$ & $\begin{array}{c}\text { Parameter Recovery } \\
(95 \% \text { CI })\end{array}$ \\
\hline \multirow{11}{*}{ Evidence } & $\begin{array}{l}\text { Rate } \\
\left(\gamma_{E, \mu}\right)\end{array}$ & - & $\begin{array}{c}.83 \\
(.77, .89) \\
\end{array}$ & $\begin{array}{c}-.01 \\
(-.05, .06) \\
\end{array}$ \\
\hline & \multirow{6}{*}{$\begin{array}{l}\text { Sensitivity } \\
\quad\left(\gamma_{E, \zeta}\right)\end{array}$} & $0 \%$ & $\begin{array}{c}0 \\
(-.11, .12)\end{array}$ & $\begin{array}{c}0 \\
(-.12, .08)\end{array}$ \\
\hline & & $3.2 \%$ & $\begin{array}{c}.36 \\
(.24, .49)\end{array}$ & $\begin{array}{c}0 \\
(-.12, .09)\end{array}$ \\
\hline & & $6.4 \%$ & $\begin{array}{c}.80 \\
(.66, .97)\end{array}$ & $\begin{array}{c}-.01 \\
(-.14, .12)\end{array}$ \\
\hline & & $12.8 \%$ & $\begin{array}{c}1.55 \\
(1.37,1.72)\end{array}$ & $\begin{array}{c}-.03 \\
(-.21, .07)\end{array}$ \\
\hline & & $25.6 \%$ & $\begin{array}{c}2.72 \\
(2.45,3.01)\end{array}$ & $\begin{array}{c}-.01 \\
(-.29, .17)\end{array}$ \\
\hline & & $51.2 \%$ & $\begin{array}{c}4.22 \\
(3.82,4.64)\end{array}$ & $\begin{array}{c}-.03 \\
(-.46, .33)\end{array}$ \\
\hline & $\underset{\left(\alpha_{E, \mu}\right)}{\text { Threshold }}$ & - & 1 & - \\
\hline & $\begin{array}{c}\text { Bias } \\
\left(\alpha_{E, \zeta}\right)\end{array}$ & - & $\begin{array}{c}-.01 \\
(-.03, .02)\end{array}$ & $\begin{array}{c}0 \\
(-.01, .02) \\
\end{array}$ \\
\hline & $\begin{array}{c}\text { Variability } \\
\left(\sigma_{E}\right)\end{array}$ & - & $\begin{array}{c}.49 \\
(.46, .52)\end{array}$ & $\begin{array}{c}-.01 \\
(-.05, .03)\end{array}$ \\
\hline & Non-decision time & - & $\begin{array}{c}.09 \\
(.07, .11)\end{array}$ & $\begin{array}{c}0 \\
(-.02, .01)\end{array}$ \\
\hline \multirow{4}{*}{ Timing } & $\begin{array}{c}\text { Rate } \\
\left(\gamma_{T}\right)\end{array}$ & - & $\begin{array}{c}1.05 \\
(1.01,1.08) \\
\end{array}$ & $\begin{array}{c}0 \\
(-.01, .04)\end{array}$ \\
\hline & $\begin{array}{c}\text { Threshold } \\
\left(\alpha_{T}\right)\end{array}$ & - & 1 & - \\
\hline & $\begin{array}{c}\text { Variability } \\
\left(\sigma_{T}\right)\end{array}$ & - & $\begin{array}{c}.22 \\
(.21, .23)\end{array}$ & $\begin{array}{l}.01 \\
(0, .04)\end{array}$ \\
\hline & $\underset{\left(\tau_{T}\right)}{\text { Onset }}$ & - & .05 & - \\
\hline
\end{tabular}


terminated many of the harder decisions, and the correct accumulator (with a higher drift rate) becomes more likely to cross threshold first for the easier decisions. Interestingly, the relative probability of a timing response increased near the relevant deadline: $1.3 \mathrm{~s}$ for the explicit deadlines and the average 1s duration of the reward delay for the implicit deadlines. The TRDM predicted CAFs capture the main trends in the observed data.

We can also test the external validity of the timing process against a feature of Roitman and Shadlen's (2002) experimental design. If the monkeys were sensitive to the duration of the reward delay then the time-to-threshold of their internal timer ought to be calibrated to the time the reward became available in the experiment. A simple hypothesis is that the expected time-to-threshold for monkey B's timing process will be faster than monkey N's timing process, since the reward delay was set to a shorter duration for monkey B (800ms) than monkey N (1200ms; for details see Roitman \& Shadlen, 2002, p.9476). The hypothesis was supported by posterior predictive data. When considering the estimates for the timing process in isolation, the mean time-to-threshold for monkey B was $893 \mathrm{~ms}(95 \%$ CI [852, 941]) and for monkey $\mathrm{N}$ was $1026 \mathrm{~ms}$ [1005, 1049]. This was a reliable difference (133ms [82, 182]) that supports external validity: parameter estimates of the TRDM's timing process are associated with relevant time-based manipulations in the experimental design. We provide further evidence of the validity of the timing process in Part 3 of the paper.

Discussion. We demonstrated that data from time-controlled paradigms previously thought to require dynamic adjustments to evidence thresholds can be accommodated by the TRDM. The TRDM account requires no modification to model architecture from the information-controlled account, whereas there is little evidence to support any change of thresholds in information-controlled paradigms (Hawkins, Forstmann, et al., 2015). The timing process of the TRDM prevents the decision maker from committing too much time to any individual choice. Decision makers appear to calibrate their timing process to allow easy (high-drift) decisions to terminate in the evidence process while cutting off (truncating) some difficult (low-drift) decisions that would otherwise require a great deal of time to cross an evidence threshold with little gain in accuracy.

\section{Time-Controlled Choice: Multiple Explicit Deadlines}

To investigate the time course of information processing, some tasks interrupt some or all of the decision trials with a 'response signal' that indicates to the participant they must respond immediately. The response signal typically takes on a range of lags across trials. Response signal tasks have been highly instructive in evaluating the time course of information processing (e.g., Gao, Tortell, \& McClelland, 2011; McElree, 2001; McElree \& Dosher, 1989), and have been previously explained in terms of pure evidence accumulation processing (e.g., Gao et al., 2011; Rae et al., 2014; Ratcliff, 2006). Here we test whether the timing process of the TRDM is still required, or whether it hinders the model, in decision contexts where processing is interrupted with multiple explicit deadlines. We assume that the TRDM operates in response-signal tasks in the same manner as it operates in typical decision-making tasks, with the addition that the externally imposed response signal can interrupt and terminate processing. When a response signal occurs prior to the evidence accumulation or timing processes reaching their respective thresholds - that is, prior to decision commitment - we assume that it has the same effect as when the latent timing 


\section{Explicit Implicit \\ Deadlines Deadlines}
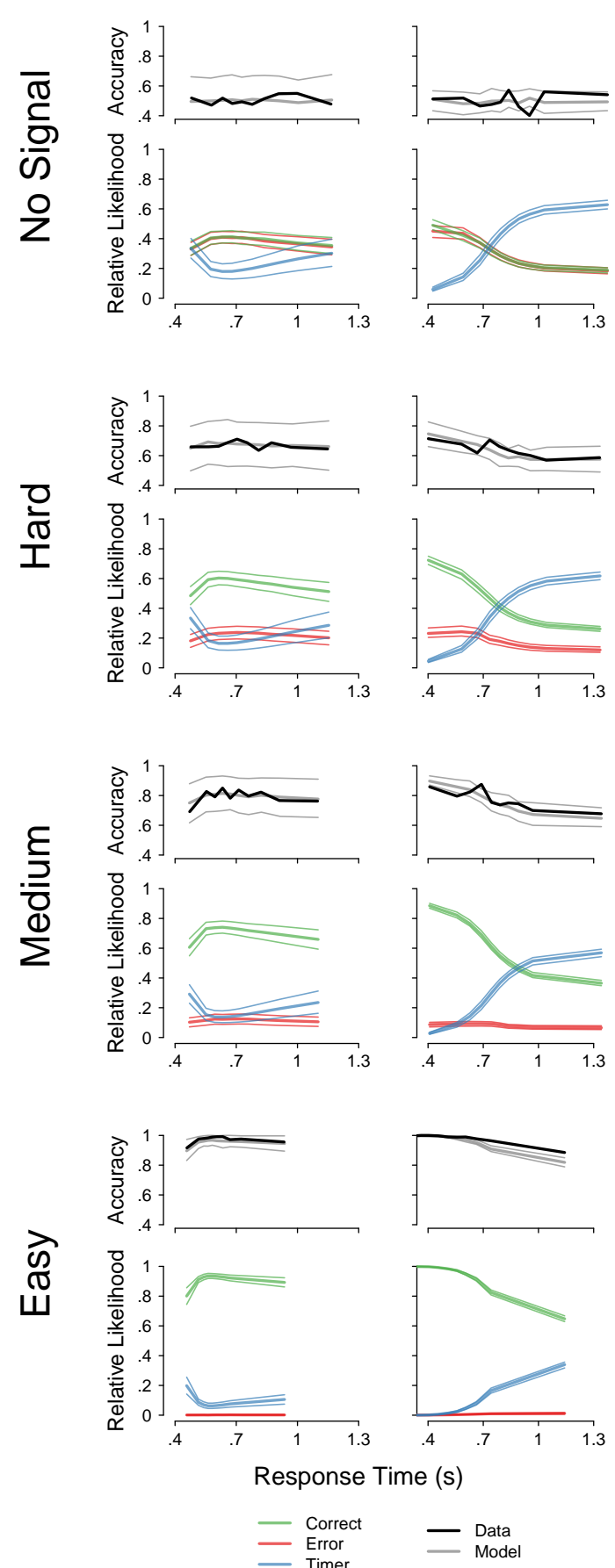

Figure 7. Posterior predicted probability of each accumulator winning the TRDM race (crossing threshold first; colored panels) and the conditional accuracy function (gray scale panels) as a function of the range of observed RTs in each experiment ( $x$-axes). Explicit deadlines (Evans, Hawkins, \& Brown, 2019) and implicit deadlines (Roitman \& Shadlen, 2002) are shown in columns. Four levels of stimulus discrimination difficulty are shown in rows. Assignment of the labels 'correct' and 'error' in the 'no signal' row is arbitrary. All other details are as described in Figure 4. 
process terminates the decision process - a stimulus-independent guess. We assume that the TRDM can terminate the decision process prior to a response signal, in which case the decision maker withholds their response until signal onset. Palestro et al. (2018) assumed a similar 'opt out' mechanism for responding prior to the response signal, which was best explained by a collapsing threshold mechanism.

We use a simulation-based approach to demonstrate the TRDM's capacity to explain typical performance in tasks with multiple explicit deadlines. The simulation was based on posterior mean parameters from the explicit deadline task of Evans, Hawkins, and Brown (2019) analyzed in the previous section (Table 6). The speed-accuracy tradeoff function was assessed across a range of response signal lags, from 0s to 1.3s; this upper limit was chosen as the time of the explicit deadline in Evans, Hawkins, and Brown (2019). Figure 8 shows that the TRDM predicts a speed-accuracy tradeoff function with the general trends that are widely observed in data: a short period of no sensitivity to stimulus information $\left(d^{\prime}=0\right)$ followed by a rapid increase in sensitivity along a negatively accelerated curve that approaches asymptote at longer lags.

We then used an optimization algorithm to estimate the best-fitting shifted exponential function to the TRDM simulated speed-accuracy tradeoff function. The shifted exponential has been shown to provide an accurate description of decision sensitivity as a function of external deadline (McElree, 2001; McElree \& Dosher, 1989). In this description the stimulus sensitivity at time $t, d^{\prime}(t)$, is

$$
d^{\prime}(t)= \begin{cases}\lambda\left(1-e^{-\beta(t-\delta)}\right), & \text { for } t>\delta \\ 0, & \text { for } t \leq \delta\end{cases}
$$

The parameters of the shifted exponential have meaningful interpretations: $\delta$ is the onset time of information processing, $\lambda$ is the asymptotic amount of processed information, and $\beta$ is the rate of approach to asymptote.

Figure 8 shows close correspondence between the TRDM speed-accuracy tradeoff function and the fit of the classic shifted exponential function; given the shifted exponential closely agrees with a range of response-signal data this provides a quantitative check on the TRDM's predictions. Table 8 shows that the parameters estimated from the shifted exponential function are all psychologically sensible: as stimulus coherence increased the exponential rate and asymptotic sensitivity grew larger but the onset time did not change. These estimates are closely related to estimates of the corresponding parameters of the TRDM: asymptotic $d^{\prime}$ sensitivity $(\lambda)$ was very similar to TRDM evidence sensitivity $\left(\gamma_{E, \zeta}\right.$; $.98,1.93,4.25$ vs $.90,1.83,4.83)$, and the onset time $(\delta)$ was very similar to TRDM nondecision time $\left(\tau_{E} ; .39, .39, .40\right.$ vs .36$) .{ }^{4}$ These results indicate that the TRDM provides an account of external deadline effects that is both descriptively accurate, psychologically plausible, and consistent with classic functions for assessing performance in the responsesignal paradigm.

\footnotetext{
${ }^{4}$ The $0 \%$ coherence condition generates a flat speed-accuracy tradeoff function so it is omitted from the comparisons.
} 


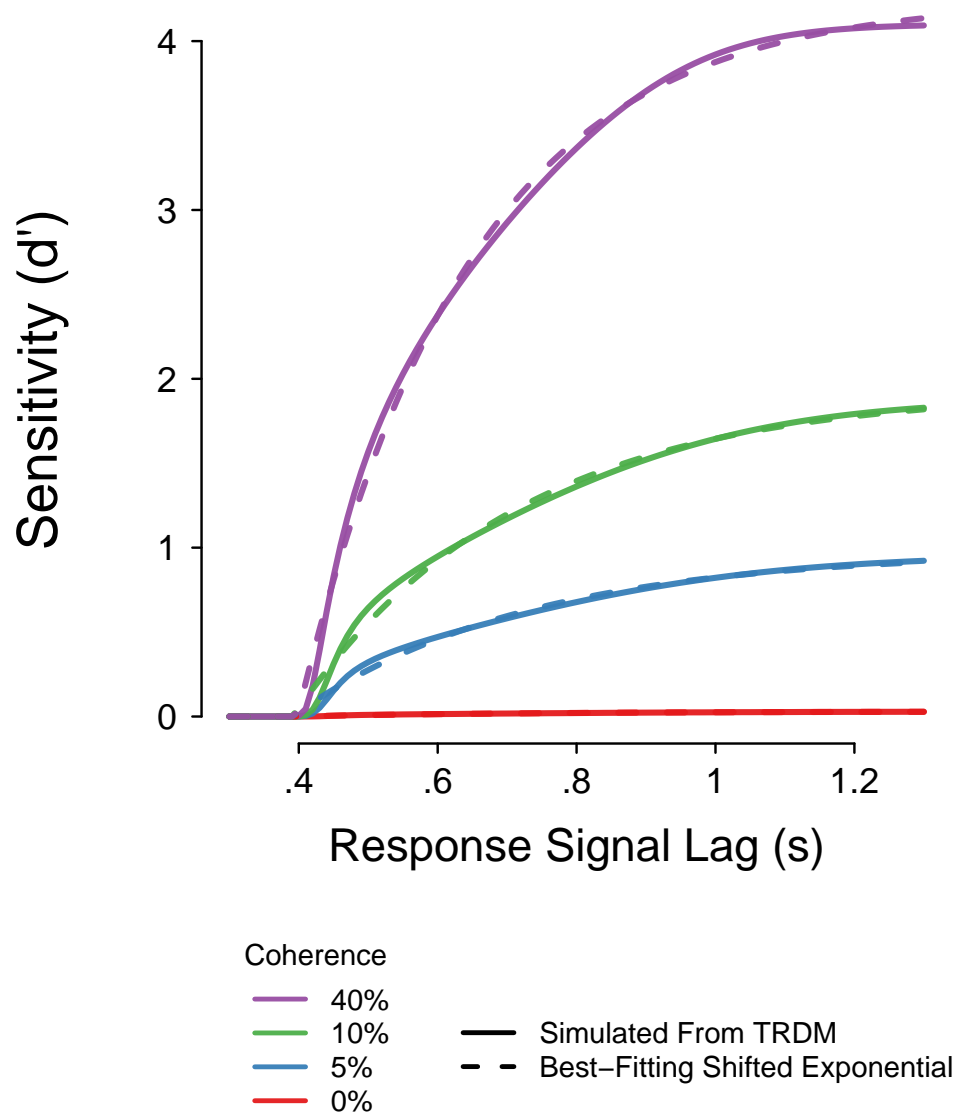

Figure 8. Speed-accuracy tradeoff function simulated from the TRDM using the posterior mean estimates from Evans, Hawkins, and Brown (2019) reported in Table 6 (solid lines) and the best-fitting shifted exponential function to those simulated data (dashed lines). Colors represent levels of motion coherence.

\section{Part 2: Implications of the TRDM's Simplifying Assumptions}

In this section we test the implications of two of the simplifying assumptions used in fitting the TRDM in the last section: no across-trial variability in parameters and unbiased guessing for responses generated by the timing process. We show that conclusions based on the TRDM are generally robust when the former assumption is violated, and that simple parameter-free adjustments can be used in paradigms where the latter assumption may not be true.

\section{Across-Trial Variability in Drift Rates}

We have argued that a strength of the TRDM is the absence of across-trial variability in model parameters. Nevertheless, in some circumstances the occurrence of such variability may be plausible. If items in a stimulus class that are all mapped to the same response differ substantially among each other in the evidence they provide for one choice vs. another then there will be across-trial variability in drift rates. In a lexical decision task, for example, 
Table 8

Parameter estimates from the shifted exponential function applied to the TRDM simulated data shown in Figure 8. 0\% coherence condition is a flat function so is omitted.

\begin{tabular}{cccc} 
Coherence & Onset $(\delta)$ & Asymptote $(\lambda)$ & Rate $(\beta)$ \\
\hline $5 \%$ & .39 & .98 & 3.03 \\
$10 \%$ & .39 & 1.93 & 3.14 \\
$40 \%$ & .40 & 4.25 & 4.06
\end{tabular}

words and non-words are heterogeneous and vary along many lexically-relevant dimensions (e.g., number of syllables, semantic content, concreteness, etc.), so they are likely to be processed differently. To take an extreme example, very low frequency words may be unknown to a particular participant and so produce evidence favoring a non-word response. Item differences, but in the context of recognition memory for words, were the initial motivation for the inclusion of across-trial variability in drift rates in the DDM (Ratcliff, 1978). However, trial-to-trial drift rate variability is also routinely assumed in applications of the DDM where there are minimal or no item differences (e.g., brightness discrimination in Ratcliff, Voskuilen, \& Teodorescu, 2018). In such cases across-trial variability in drift rate might be justified by fluctuations in attention, such as could be caused by mind wandering (Hawkins, Mittner, Forstmann, \& Heathcote, 2017). Although the latter possibility is difficult to directly test, and even in cases where there are objective item differences it would be more desirable to explicitly model them (e.g., Nosofsky \& Palmeri, 1997; Turner, 2019), we still thought it prudent to check by simulation whether ignoring systematic across-trial variability in drift rate produces biases that are likely to cause misinterpretation of the TRDM's parameter estimates.

Method. We performed a parameter recovery exercise to test the TRDM's robustness to across-trial variability in drift rate. We started with the TRDM parameters estimated from the DIC-best model of the lexical decisions in Part 1 (Wagenmakers et al., 2008). We used them to generate synthetic data with the same properties as the real data (i.e., number of trials for each stimulus, total number of trials, and number of participants) but with item effects added in the form of across-trial variability in drift rate, using the standard normal form (Ratcliff, 1978): drift rate $\gamma$ on trial $i$ in condition $j$ distributed $\gamma_{i j} \sim N\left(\gamma_{j}, \eta\right)$, where $\eta$ is the standard deviation (SD) of the across-trial distribution of drift rates.

We generated data from the TRDM under three conditions: low, moderate and high SD for the across-trial drift rate distribution. Appropriate SD values were based on Matzke and Wagenmakers (2009), who reported mean $(\gamma)$ and SD $(\eta)$ aggregated over many studies to be $\gamma=.223$ and $\eta=.133$. This makes the mean-to-SD ratio $\frac{\gamma}{\eta}=1.68$; that is, the drift rate mean was 1.68 times larger than the drift rate $\mathrm{SD}$. We set this ratio to be our moderate variability condition $\left(\frac{\gamma}{\eta}\right.$ moderate $\left.=1.68\right)$. For the high variability condition we halved the ratio $\left(\frac{\gamma}{\eta}_{\text {high }}=.84\right)$. This means there is more variability across trials than there is mean signal (i.e., $\eta>\gamma$ ), which is quite extreme. For the low variability condition we added the first two ratios together $\left(\frac{\gamma}{\eta}_{\text {low }}=\frac{\gamma}{\eta}_{\text {moderate }}+\frac{\gamma}{\eta}_{\text {high }}=2.52\right)$. This was set so that the difference between the successive SDs were constant $\left(\frac{\gamma}{\eta}_{\text {low }}-\frac{\gamma}{\eta_{\text {moderate }}}=\frac{\gamma}{\eta}_{\text {moderate }}-\frac{\gamma}{\eta}_{\text {high }}\right)$. For comparison, we also incuded a baseline condition that did not include across-trial variability 
in drift rate (i.e., the standard TRDM with parameter recovery shown in Table 4, repeated here for comparison). We used the mean-to-SD ratios to obtain participant-specific SDs by first averaging each participant's estimated drift rate across the 4 stimulus categories (HF, LF, VLF, NW) and 2 instruction conditions (speed, accuracy) and solving for the value of $\eta$ that returned the $\frac{\gamma}{\eta}$ ratio for the condition.

The parameter recovery exercise was conducted in the same manner as for the data sets reported in Part 1 with one exception: the entire procedure outlined in Appendix D was repeated 4 times, once for each $\frac{\gamma}{\eta}$ ratio (none, low, moderate, high). That is, the sampled parameter vectors were used to simulate data with across-trial variability in correct and error drift rates. This produced a total of 10 independent hierarchical estimation exercises for each of the four variability conditions, assessing parameter recovery from a total of 680 participant-level posterior distributions ( 17 participants $\times 4$ variability conditions $\times 10$ independent repetitions).

Results. Figure 9 shows the fit that the TRDM with no across-trial variability provides to data simulated from the TRDM with varying degrees of across-trial variability in drift rates. For all summary statistics, the goodness of fit decreased as rate variability increased, as shown by the RMSE values in each panel. This is a reasonable trend given the extent of model mis-specification is an increasing function of rate variability. Nevertheless, even at high levels of drift rate variability the TRDM still provides a good account of the data by conventional standards. This suggests the TRDM does not systematically misfit data in the presence of across-trial variability in drift rate, and that misfit of the TRDM could not be used to identify the presence of across-trial variability in drift rate.

Table 9 shows the parameter recovery results in a similar format to Table 3 with the addition of the rightmost 5 columns. These show the median of the data-generating parameters ("Generating Median") and the median and $95 \%$ credible interval of the distribution of differences between the median of the participant-level posterior distributions estimated from the simulated data and the individual-participant data generating parameters. As above, 0 represents perfect recovery, positive values indicate over-estimation relative to the data-generating parameters and negative values indicate under-estimation.

The "none" column shows that parameter recovery is very good for the standard TRDM, with all distributions centred at or very close to 0 . When random variability in drift rate is added to the TRDM the parameter recovery becomes noisier as reflected in larger $95 \%$ credible intervals on estimates. This is to be expected because the addition of acrosstrial drift rate variability makes the simulated choices and RTs more variable. However, for all but one parameter there was insufficient systematic bias in recovery to cause zero to fall outside of the $95 \%$ credible interval. The exception is within-trial variability in the evidence accumulation process $\left(\sigma_{E}\right)$, which is increasingly over-estimated as across trial variability increases.

This result is consistent with Tillman et al. (in press), who showed that data generated from a decision process with increasingly larger across-trial variability in drift rate is absorbed into increasingly larger estimates of within-trial drift rate variability in a RDM (i.e., racing Walds, without a timing process). Our recovery analysis extends that of Tillman et al. (in press) who forced all across-trial drift rate variability in the simulated data to be absorbed into within-trial drift rate variability in the estimated model. Our analysis allowed for the possibility that across-trial variability in drift rate might be absorbed into 

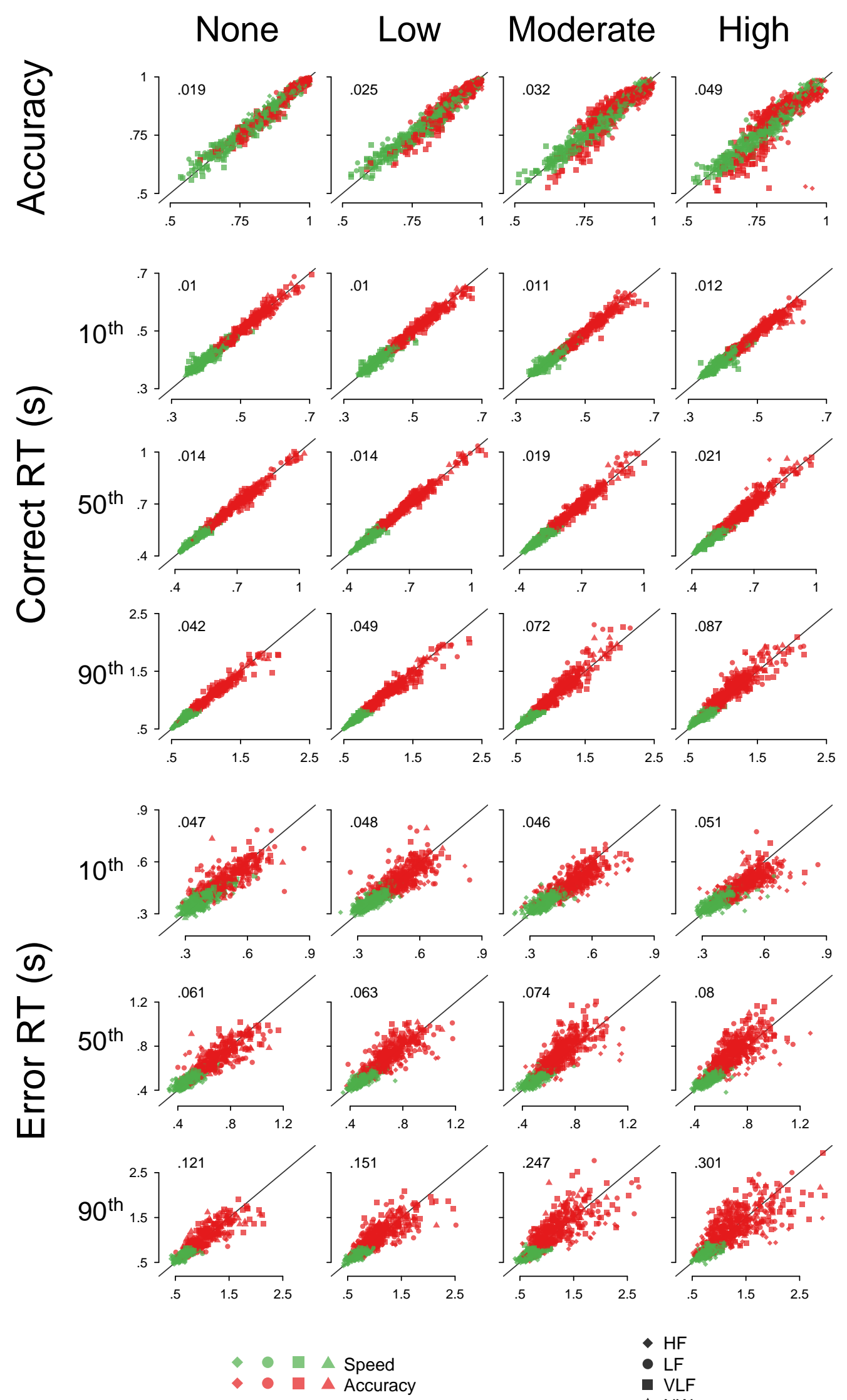

- HF

- LF

VLF

$\Delta \mathrm{NW}$

Figure 9. Simulated data for the parameter recovery exercise ( $x$-axes) vs. mean posterior predictive data from the TRDM ( $y$-axes) for individual (simulated) participants. Columns show the four levels of across-trial variability in simulated drift rates. All other details are as described in Figure 3. 
Table 9

Parameter recovery for the TRDM with varying degrees of across-trial variability in drift rate. Columns 1-4 are as described in Table 4. Column 5 shows the median data-generating parameter values. Columns 6-9 show the four levels of across-trial variability in drift rate - none, low, moderate, high - with cell entries showing the median of the difference distribution between the median of the participant-level posterior distributions estimated from the simulated data and the data-generating parameters, with underset values showing the 95\% credible interval. Positive values indicate over-estimation of a parameter and negative values indicate under-estimation of a parameter, relative to the data-generating values.

\begin{tabular}{|c|c|c|c|c|c|c|c|c|}
\hline \multirow{2}{*}{ Process } & \multirow{2}{*}{ Parameter } & \multirow{2}{*}{ Emphasis } & \multirow{2}{*}{ Stimulus } & \multirow{2}{*}{$\begin{array}{c}\text { Generating } \\
\text { Median }\end{array}$} & \multicolumn{4}{|c|}{ Estimated } \\
\hline & & & & & None & Low & Moderate & High \\
\hline \multirow{16}{*}{ Evidence } & \multirow{2}{*}{$\begin{array}{l}\text { Rate } \\
\left(\gamma_{E, \mu}\right)\end{array}$} & Speed & - & 1.87 & $\begin{array}{c}-.01 \\
(-.22, .20)\end{array}$ & $\begin{array}{c}-.03 \\
(-.38, .26)\end{array}$ & $\begin{array}{c}-.09 \\
(-.63, .30)\end{array}$ & $\begin{array}{c}-.17 \\
(-.94, .30)\end{array}$ \\
\hline & & Accuracy & - & 1.09 & $\begin{array}{c}.03 \\
(-.31, .27)\end{array}$ & $\begin{array}{c}-.17 \\
(-.64, .15)\end{array}$ & $\begin{array}{c}-.51 \\
(-1.22,-.05)\end{array}$ & $\begin{array}{c}-.63 \\
(-1.32,-.02)\end{array}$ \\
\hline & \multirow{8}{*}{$\underset{\left(\gamma_{E, \zeta}\right)}{\text { Sensitivity }}$} & \multirow{4}{*}{ Speed } & $\mathrm{HF}$ & 6.31 & $\begin{array}{c}-.21 \\
(-1.32, .88)\end{array}$ & $\begin{array}{c}-.21 \\
(-1.54,1.39)\end{array}$ & $\begin{array}{c}-.05 \\
(-1.50,1.16)\end{array}$ & $\begin{array}{c}-.06 \\
(-1.88,2.63)\end{array}$ \\
\hline & & & LF & 3.37 & -.17 & -.07 & -.10 & -.19 \\
\hline & & & VLF & 1.89 & $\begin{array}{c}(-.80, .59) \\
-.01 \\
(-.65, .86)\end{array}$ & $\begin{array}{c}(-.89, .85) \\
-.07 \\
(-.86, .80)\end{array}$ & $\begin{array}{c}(-.86,1.00) \\
-.01 \\
(-.90, .91)\end{array}$ & $\begin{array}{c}(-1.16, .99) \\
-.04 \\
(-.85, .89)\end{array}$ \\
\hline & & & NW & 4.10 & $\begin{array}{c}.10 \\
(-.89, .67)\end{array}$ & $\begin{array}{c}-.08 \\
(-.84, .72)\end{array}$ & $\begin{array}{c}-.01 \\
(-.94,1.04)\end{array}$ & $\begin{array}{c}-.20 \\
(-.92,1.22)\end{array}$ \\
\hline & & \multirow{4}{*}{ Accuracy } & $\mathrm{HF}$ & 5.16 & $\begin{array}{c}-.12 \\
(-1.58, .91)\end{array}$ & $\begin{array}{c}-.08 \\
(-1.41,1.04)\end{array}$ & $\begin{array}{c}-.18 \\
(-1.57,1.17)\end{array}$ & $\begin{array}{c}-.61 \\
(-2.50, .98)\end{array}$ \\
\hline & & & LF & 3.19 & -.08 & -.12 & -.35 & -.74 \\
\hline & & & VLF & 1.95 & -.08 & -.16 & -.38 & -.65 \\
\hline & & & NW & 3.02 & $\begin{array}{c}-.001 \\
-.01 \\
(-.69, .53)\end{array}$ & $\begin{array}{c}(-.64, .41) \\
-.07 \\
(-.73, .57)\end{array}$ & $\begin{array}{c}(-1.18, .37) \\
-.31 \\
(-1.27, .39)\end{array}$ & $\begin{array}{c}(-1.42,-.03) \\
-.73 \\
(-1.60, .17)\end{array}$ \\
\hline & \multirow{2}{*}{$\underset{\left(\alpha_{E, \mu}\right)}{\text { Threshold }}$} & Speed & - & 1 & - & - & - & - \\
\hline & & Accuracy & - & 1.05 & $\begin{array}{c}0 \\
(-.19, .21) \\
\end{array}$ & $\begin{array}{c}-.06 \\
(-.25, .15)\end{array}$ & $\begin{array}{c}-.14 \\
(-.42, .02)\end{array}$ & $\begin{array}{c}-.19 \\
(-.49, .05)\end{array}$ \\
\hline & $\begin{array}{c}\text { Bias } \\
\left(\alpha_{E, \zeta}\right) \\
\end{array}$ & - & - & .02 & $\begin{array}{c}.01 \\
(-.06, .09) \\
\end{array}$ & $\begin{array}{c}.01 \\
(-.08, .08)\end{array}$ & $\begin{array}{c}.01 \\
(-.07, .09) \\
\end{array}$ & $\begin{array}{c}0 \\
(-.08, .10) \\
\end{array}$ \\
\hline & $\begin{array}{c}\text { Variability } \\
\left(\sigma_{E}\right)\end{array}$ & - & - & 1.09 & $\begin{array}{c}0 \\
(-.19, .13)\end{array}$ & $\begin{array}{c}.01 \\
(-.15, .14)\end{array}$ & $\begin{array}{c}.16 \\
(-.06, .31)\end{array}$ & $\begin{array}{c}.30 \\
(.06, .67)\end{array}$ \\
\hline & \multirow{2}{*}{$\underset{\left(\tau_{E}\right)}{\mathrm{NDT}}$} & Speed & - & .31 & $\begin{array}{c}0 \\
(-.02, .02)\end{array}$ & $\begin{array}{c}0 \\
(-.02, .02)\end{array}$ & $\begin{array}{c}.01 \\
(-.01, .02)\end{array}$ & $\begin{array}{c}.01 \\
(0, .03)\end{array}$ \\
\hline & & Accuracy & - & .36 & $\begin{array}{c}0 \\
(-.03, .02) \\
\end{array}$ & $\begin{array}{c}0 \\
(-.02, .04) \\
\end{array}$ & $\begin{array}{c}.03 \\
(0, .07)\end{array}$ & $\begin{array}{c}.04 \\
(0, .09) \\
\end{array}$ \\
\hline \multirow{5}{*}{ Timing } & \multirow{2}{*}{$\begin{array}{c}\text { Rate } \\
\left(\gamma_{T}\right)\end{array}$} & Speed & - & 1.13 & $\begin{array}{c}0 \\
(-.28, .22)\end{array}$ & $\begin{array}{c}.02 \\
(-.31, .28)\end{array}$ & $\begin{array}{c}.01 \\
(-.44, .34)\end{array}$ & $\begin{array}{c}0 \\
(-.46, .44)\end{array}$ \\
\hline & & Accuracy & - & .24 & $\begin{array}{c}0 \\
(-.25, .16) \\
\end{array}$ & $\begin{array}{c}.14 \\
(-.24, .55) \\
\end{array}$ & $\begin{array}{c}.30 \\
(-.17, .63) \\
\end{array}$ & $\begin{array}{c}.37 \\
(-.22, .76) \\
\end{array}$ \\
\hline & $\begin{array}{c}\text { Threshold } \\
\left(\alpha_{T}\right)\end{array}$ & - & - & 1 & - & - & - & - \\
\hline & $\begin{array}{c}\text { Variability } \\
\left(\sigma_{T}\right)\end{array}$ & - & - & .53 & $\begin{array}{c}0 \\
(-.05, .05) \\
\end{array}$ & $\begin{array}{c}-.01 \\
(-.07, .05) \\
\end{array}$ & $\begin{array}{c}0 \\
(-.06, .06) \\
\end{array}$ & $\begin{array}{c}.01 \\
(-.07, .09) \\
\end{array}$ \\
\hline & $\underset{\left(\tau_{T}\right)}{\text { Onset }}$ & - & - & .05 & - & - & - & - \\
\hline
\end{tabular}


any of the estimated TRDM parameters, and we still find that the strongest systematic effect is that it is absorbed into within-trial drift rate variability.

There were also some weaker bias trends in the parameter recovery. For example, the recovered mean rates of evidence accumulation $\left(\gamma_{E, \mu, \text { Speed }}\right.$ and $\left.\gamma_{E, \mu, A c c}\right)$ tended to be lower than the generating values, at least at high variability. There was also a correspondingly small underestimation of drift rate sensitivity parameters $\left(\gamma_{E, \zeta .}\right)$, and a slight tendency to overestimate non-decision time in the evidence process $\left(\tau_{E}\right)$.

In summary, we argue that it is only important to include across-trial variability in drift rate in the TRDM if it occurs at very high levels. Otherwise, we believe the advantages of assuming no across-trial variability in rates outweigh the potential for confounding in the interpretation of the TRDM's parameter estimates. Where it is likely that one condition might have substantially greater across-trial variability than another, differences in withintrial variability might instead be interpreted as due to across-trial variability. Secondarily, greater across-trial variability might be the cause of an increase in non-decision time or a reduction in means and sensitivities of evidence rates. However, our simulation result shows differences in across-trial variability would likely have to be extreme to be of practical concern. More generally, our results support the possibility that across-trial variability in drift rates has been widely incorporated in evidence accumulation models because it absorbs variance caused by the combined effect of within-trial variability and a timing process (see O'Connell, Shadlen, Wong-Lin, \& Kelly, 2018, for a related argument that collapsing thresholds can replace across-trial variability in the DDM as an explanation of slow errors).

\section{Timing-Based Guessing Policies}

We have assumed that when the timing process terminates the decision process then the response is chosen by an unbiased guess. That is, for a choice between $N$ options the timing-based guessing probabilities for options $n=1, \ldots, N$ are $g_{n}=\frac{1}{N}$, so, for example, $g_{1}$ is the probability that a stimulus-uninformed guess favors option 1 . This is the simplest possible guessing assumption, though many others are possible. For instance, the guessing probability could be informed by the state of the evidence accumulation process at time $t$, conditioned on the evidence process not terminating prior to $t$ ("partial information", see Ratcliff, 1980, 1988, 2006). We did not investigate access to partial information in the TRDM for two reasons. First, we found that the simpler assumption of stimulus-uninformed guessing provided an excellent account of all data sets we examined. As mentioned earlier, this is not to say that we believe people never have access to partial information, just that we did not find evidence it was required in our data. Second, there is no closed-form solution for access to partial information in a racing Wald architecture. This means we would no longer obtain an analytic solution to the TRDM.

We investigated four variants of the TRDM with different guessing policies, none of which rely on access to the state of the evidence process, and each of which supports good parameter recovery. We show that different TRDM assumptions for timing-based guesses do not dramatically alter the explanation of data. We tested the four guessing-variants in a design with different base rates for stimuli corresponding to each response (Experiment 2 of Wagenmakers et al., 2008). This manipulation produces a strong bias in favor of the majority stimulus (Heathcote, Holloway, \& Sauer, 2019; Mulder, Wagenmakers, Ratcliff, 
Boekel, \& Forstmann, 2012). The bias is most often explained as due to a reduced distance from the start point to threshold for the majority stimulus than for the minority stimulus (White \& Poldrack, 2014) although in extreme manipulations there is also evidence that it can result in a fast guess in favor of the majority stimulus (Noorbaloochi, Sharon, \& McClelland, 2015; Simen et al., 2009). We tested whether the classic explanation based on strategic threshold adaptation in the evidence process is sufficient for the TRDM to explain the data, or if biased guessing policies are required in the timing process.

Method. Experiment 2 of Wagenmakers et al. (2008) was methodologically similar to their Experiment 1 (i.e., the lexical decision experiment addressed in Part 1 of this paper) except that a stimulus bias manipulation was substituted for the speed-accuracy tradeoff manipulation. In alternating blocks of trials, 19 participants made decisions about stimuli composed of $75 \%$ words and $25 \%$ non-words, or $75 \%$ non-words and $25 \%$ words. All blocks included high, low and very low frequency words, and non-words. For complete experimental details we refer the reader to the primary source.

Figure 10 shows the strong response bias effects observed in Wagenmakers et al.'s (2008) data. The $x$-axes show the bias for a word response for individual participants: the proportion correct for blocks with majority words minus the proportion correct for blocks with majority non-words. Positive values therefore indicate a bias to respond 'word' and negative values indicate a bias to respond 'non-word'. The three word stimulus classes have positive values, particularly for low and very low frequency words, while the non-word stimulus class has negative values.

We formalize the four guessing-variants of the TRDM with notation where the guessing probability of a response from the timing process $g$ has a two-term subscript: the first term describes the majority stimulus of a block (words $-w$, non-words $-n w$ ) and the second term describes the response (word $-W$, non-word $-N W$ ). The first TRDM variant is the standard TRDM with pure guessing for responses terminated by the timing process: $g_{(w, W)}=g_{(w, N W)}=.5$, and $g_{(n w, W)}=g_{(n w, N W)}=.5$. The second variant assumes a biased guessing probability that is freely estimated from data: $g_{(w, W)} \sim[0,1]$, and $g_{(w, N W)}=1-g_{(w, W)}$. We made the simplifying assumption that word and non-word responses biases are of equivalent magnitude; $g_{(n w, N W)}=g_{(w, W)}$, and $g_{(n w, W)}=1-g_{(n w, N W)}$. The bias probability has no theoretical motivation which makes this a descriptive model, though it serves as a useful "most-flexible" comparison (i.e., it should have the best overall performance as the other models are special cases). The third variant assumes that guessing proportions are set based on perfect knowledge of the bias proportions in the experimental design: $g_{(w, W)}=g_{(n w, N W)}=.75$ and $g_{(w, N W)}=g_{(n w, W)}=.25$. This variant approximates a process where participants learn the base rates of the stimulus categories and use these as their timing-based guessing probabilities, which is analogous to probability matching.

The fourth variant has a fully specified psychological-process motivation, mapping the magnitude of the evidence thresholds from the evidence accumulation process into a guessing probability. We choose a simple parameter-free mapping: in majority word blocks, the difference in evidence thresholds for a word response $\left(\alpha_{E, w, W}\right)$ and a non-word response $\left(\alpha_{E, w, N W}\right)$ relative to the overall height of thresholds. Hence, the guessing probability that a timing response favors a word response is $g_{(w, W)}=1-\frac{\alpha_{E, w, W}}{\alpha_{E, w, W}+\alpha_{E, w, N W}}$, and $g_{(w, N W)}=$ $1-g_{(w, W)}$. Similarly, for majority non-word blocks we have $g_{(n w, N W)}=1-\frac{\alpha_{E, n w, N W}}{\alpha_{E, n w, N W}+\alpha_{E, n w, W}}$ and $g_{(n w, W)}=1-g_{(n w, N W)}$. This variant does not assume the timing process has access 


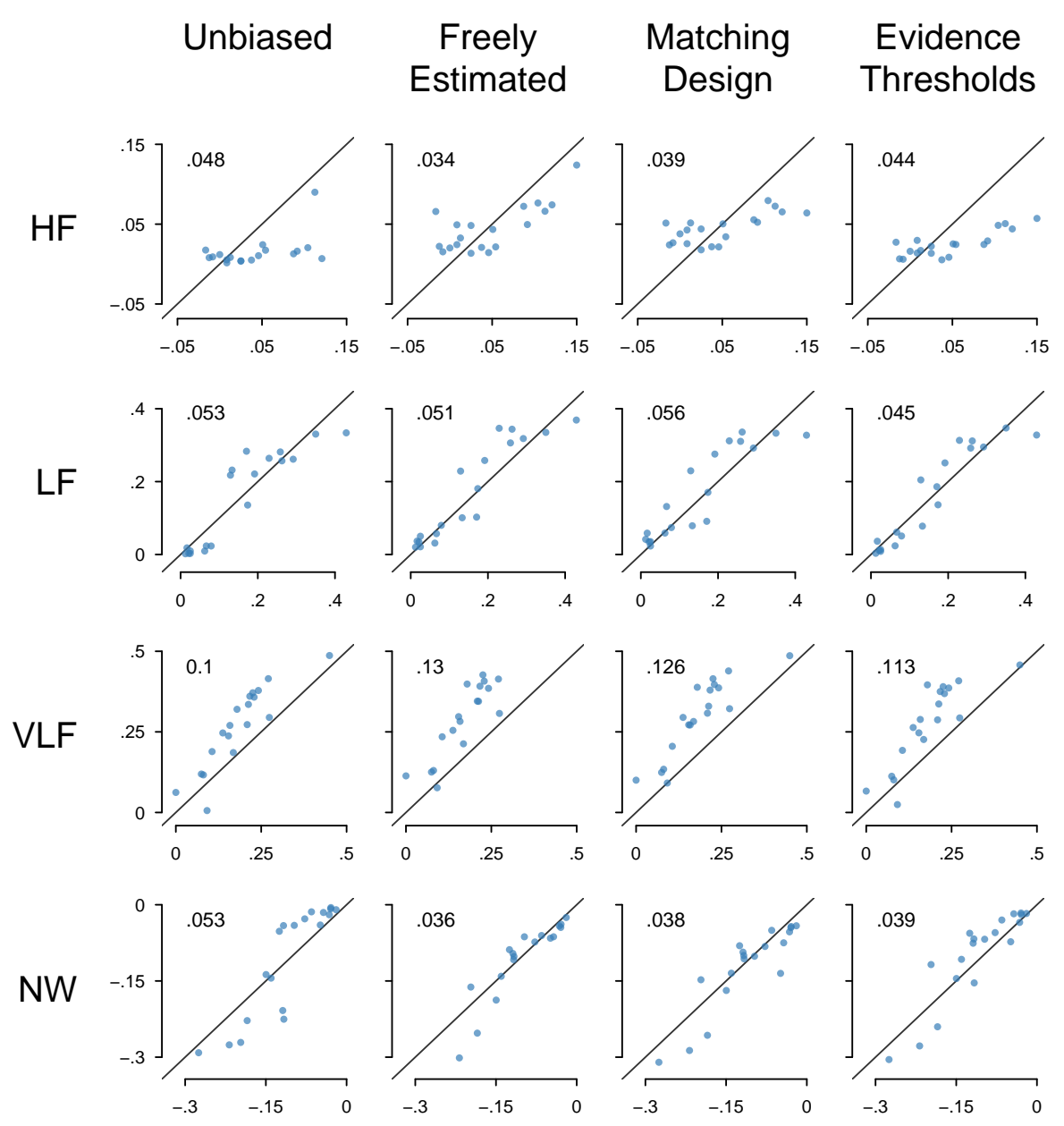

Figure 10. Observed ( $x$-axes) vs. predicted (y-axes) response bias for individual participants. Rows represent the four word frequency categories investigated in Experiment 2 of Wagenmakers et al. (2008). Columns represent the four guessing-variants of the TRDM (see main text for details). The symbols represent the proportion correct for majority word blocks - majority non-word blocks; positive values indicate a bias to respond 'word' and negative values indicate a bias to respond 'non-word'. The value in the upper left of each panel shows the root mean square error. 
to the partial state of the evidence accumulation process. Rather, it assumes the decision maker has knowledge of the level of a priori bias in the evidence accumulation process (i.e., strategic threshold settings) and this level of psychological bias is used to inform the guessing probability of the timing process.

We crossed the four TRDM guessing variants with a second factor: whether evidence thresholds in the evidence accumulation process are freely estimated for each response (word, non-word) or constrained to a common value. This tests whether the conventional explanation of asymmetric evidence thresholds for biased responding are still necessary in the TRDM. When evidence thresholds were constrained to be the same across evidence accumulators, the TRDM performed much more poorly - the DIC difference to the best performing models was over 4,000 units, so we do not report the equal-threshold models further.

Results. Table 10 shows zero-referenced DIC values for the four guessing variants of the TRDM. The model with freely estimated response bias provides the best explanation of the data, but has the drawback that it lacks any psychological motivation. However, Figure 10, which shows observed vs. posterior predicted choice proportions for individual participants, suggests that it offers only a negligible improvement over the parameter-free models. The choice proportion predictions are even similar across the two most distinct guessing variants (unbiased, freely estimated), despite the $\Delta$ DIC values in Table 10. Indeed, there is a very strong correlation between posterior predicted choice proportions of the freely-estimated and unbiased guessing variants, $r=.966$. The correlation was even stronger between the freely-estimated and evidence-thresholds variants, $r=.992$. This suggests that a parameter-free transformation of the evidence thresholds can usefully inform a biased probability of guessing for timing responses in the TRDM. Nevertheless, maintaining an assumption of unbiased guessing did not lead to a very noticeable decrease in the fit to choice data. This was also true for the RT data: Figure 1 in the supplementary material shows very similar posterior predictive RTs across the two most distinct guessing variants with no systematic differences in goodness of fit.

Table 10

DIC model comparison for four guessing-variants of the TRDM for Experiment 2 from Wagenmakers et al. (2008). Column 2 shows the number of model parameters freely estimated for each participant. Column 3 shows DICs zero-referenced to the DIC-preferred model such that positive values indicate a poorer explanation of the data. Notation is simplified for clarity; see main text for complete details.

\begin{tabular}{lcc} 
Guessing variant & $\begin{array}{c}\text { Number of } \\
\text { Free Parameters }\end{array}$ & $\Delta$ DIC \\
\hline Unbiased $\left(g_{1}=.5\right)$ & 12 & 199 \\
Bias freely estimated $\left(g_{1} \sim[0,1]\right)$ & 13 & 0 \\
Bias matching experimental design $\left(g_{1}=.75\right)$ & 12 & 75 \\
Bias derived from evidence thresholds $\left(g_{1}=1-\frac{\alpha_{E, 1}}{\alpha_{E, 1}+\alpha_{E, 2}}\right)$ & 12 & 89
\end{tabular}

Explaining performance in bias manipulation experiments warrants further, targeted investigation with the TRDM. However, the present results indicate that even in a base-rate manipulation experiment that produced strong bias the simple unbiased-guessing version of 
the TRDM is sufficient to produce a quite accurate account. There is weak evidence that the TRDM may require some form of biased guessing process in such bias manipulation tasks, in which case both parameter-free options offered a similar account. Bias matching the design is one option, but leaves open the question of how participants learn the required values. Transformation of the evidence thresholds also implicitly assumes some form of learning, but is more parsimonious in that the same learning process supports settings for both thresholds and bias.

\section{Part 3: Beyond Standard Choice Tasks}

In this section we test the capacity of the TRDM to explain two behavioral phenomena beyond the range of standard evidence accumulation models: high rates of pre-emptive responding and the relationship between individual differences in timing ability and decision making.

\section{Pre-Emptive Responses}

Hawkins et al. (2019) recently demonstrated that some contexts are particularly conducive to repetitive and rapid responding, which poses significant challenges for evidence accumulation models. They examined performance in the SART, a go-nogo task with a simple choice stimulus and a very high proportion of 'go' trials that induces what appears to be fast rhythmic (i.e., time-based) responding on some trials. These preemptive rhythmic responses produce a pattern of data that cannot be accommodated by conventional evidence accumulation models: very fast responses $(<.15 \mathrm{~s})$, a shallow leading edge of the RT distribution, and error RT distributions that are much faster than correct RT distributions.

To account for those data, Hawkins et al. (2019) developed a hybrid time-based and evidence-accumulation based model, the rhythmic race model (RRM). The RRM has structural similarities to the TRDM: a decision process races against an unconditionallytriggered 'runner' that we hypothesized is calibrated to the timing properties of the task. In the RRM the finishing time for this runner has a very flexible Weibull distributional form determined by three estimated parameters. Although this flexibility means that the RRM provides a very accurate description of SART data, the Weibull distribution lacks a process-based motivation, so its parameters do not have a clear psychological interpretation. We propose that the TRDM, although less flexible as its timing component has only two estimated parameters, provides an alternative, more psychologically motivated, explanation of the preemptive responding observed in the go-nogo tasks of Hawkins et al. (2019).

Method. We re-analyzed the data from Experiment 1 of Hawkins et al. (2019). The task involved recognition of single digits with a go vs. nogo response rule: withhold a response if a 3 is shown, and press a button when any other digit is shown (1-2, 4-9). Digits were uniformly sampled so that 8 in every 9 trials, on average, required a button press response. 19 participants each completed 640 go trials and 80 nogo trials.

The trial timeline commenced with a fixation cross shown for .25 seconds, followed by the digit stimulus for the trial shown for .25 seconds, followed by a blank display shown for .9 seconds. The participant could respond from the time of digit stimulus onset. Once the .9 second period of blank display elapsed, the next trial commenced with the fixation cross. This means the trial timeline was constant across trials. Although the response window was 
relatively short, we note that all responses from all participants were considerably faster than the 1.15 second response window (see $x$-axes of Figure 11).

We estimated the TRDM using identical methods as in previous applications with two exceptions. First, the onset time for the timing process was set to $\tau_{T}=0$, which mirrors the RRM setup in Hawkins et al. (2019). This is a reasonable assumption as all elements of the trial timeline were perfectly predictable: there was no variability in the stimulus display time or the inter-stimulus interval across trials. Second, we did not use the mean-sensitivity parameterization for drift rates in the evidence process. Rather, to facilitate comparison with the RRM we followed Hawkins et al.'s (2019) approach with separate evidence rates for go responses to go stimuli (denoted $\left.\gamma_{E, g o \mid g o}\right)$, go responses to nogo stimuli $\left(\gamma_{E, \text { go } \mid \text { nogo }}\right)$, and nogo responses to nogo stimuli $\left(\gamma_{E, \text { nogo|nogo }}\right) .^{5}$ There were so few nogo responses to go stimuli that we made the simplifying assumption $\gamma_{E, \text { nogo|go }}=0$, following Hawkins et al. (2019). Both the TRDM and RRM architectures assume one evidence accumulator corresponding to go responses and another corresponding to nogo responses. In both cases unbiased thresholds set at 1 were assumed to make the models identifiable. Appendix B has complete details of the parameterization.

Results. To facilitate comparison across studies, Figure 11 shows the posterior predictive distribution of the TRDM in a format that mirrors Hawkins et al.'s (2019) Figures 5 and 6 . The TRDM captures the key features in data: a substantial proportion of very fast responses $(<.15 \mathrm{~s})$, and RT distributions that are almost Gaussian for some participants. Although the RRM provided a better explanation of the data than the TRDM by a substantial margin of 1,229 DIC units, comparison of Figure 11 with Hawkins et al. (2019) reveals very little noticeable improvement in fit. We believe the advantage in DIC is most likely because the RRM was designed to explain data in this specific go-nogo task, whereas the TRDM is designed as a domain-general model of choice. This difference in scope means the TRDM is unlikely to provide as precise an explanation of data as domain-specific models, though its generality allows it to explain a broader range of data.

Reassuringly, there are convergent conclusions for the parameters common to both models. Table 11 shows there are no clear differences among the population means of the three estimated drift rates $\left(\gamma_{E, g o \mid g o}, \gamma_{E, g o \mid \text { nogo }}, \gamma_{E, \text { nogo|nogo }}\right)$ as indicated by overlapping 95\% CIs for the population mean distribution estimates; Hawkins et al. (2019) observed the same pattern. The estimated non-decision times of the evidence process are also very similar between models (TRDM: .13s, 95\% CI [.11, .16], RRM: .12s [.11, .14]).

However, the models also have important differences in their explanation for the unusual patterns in RT data. The RRM proposes that participants form a repetitive pattern of responding to protect against the negative side effects that mind wandering can have on responding (i.e., failing to respond on go trials). It generates a distribution of timed responding that mimics the shape and location of the distribution of anticipatory responses. To do so the shape parameter of the Weibull plays a crucial role, but that parameter has

\footnotetext{
${ }^{5}$ Drift rates for nogo responses $\left(\gamma_{E, \text { nogo|. }}\right)$ are identifiable in principle even though there is no response, because observing no response provides information about the outcome of the decision process. When the nogo accumulator reaches threshold before the go accumulator, the model generates a 'withhold' response (i.e., no overt behavioural response is observed). The proportion of withheld responses constrains the magnitude of the difference between the drift rates for go and nogo accumulators, relative to the variability of the accumulators.
} 


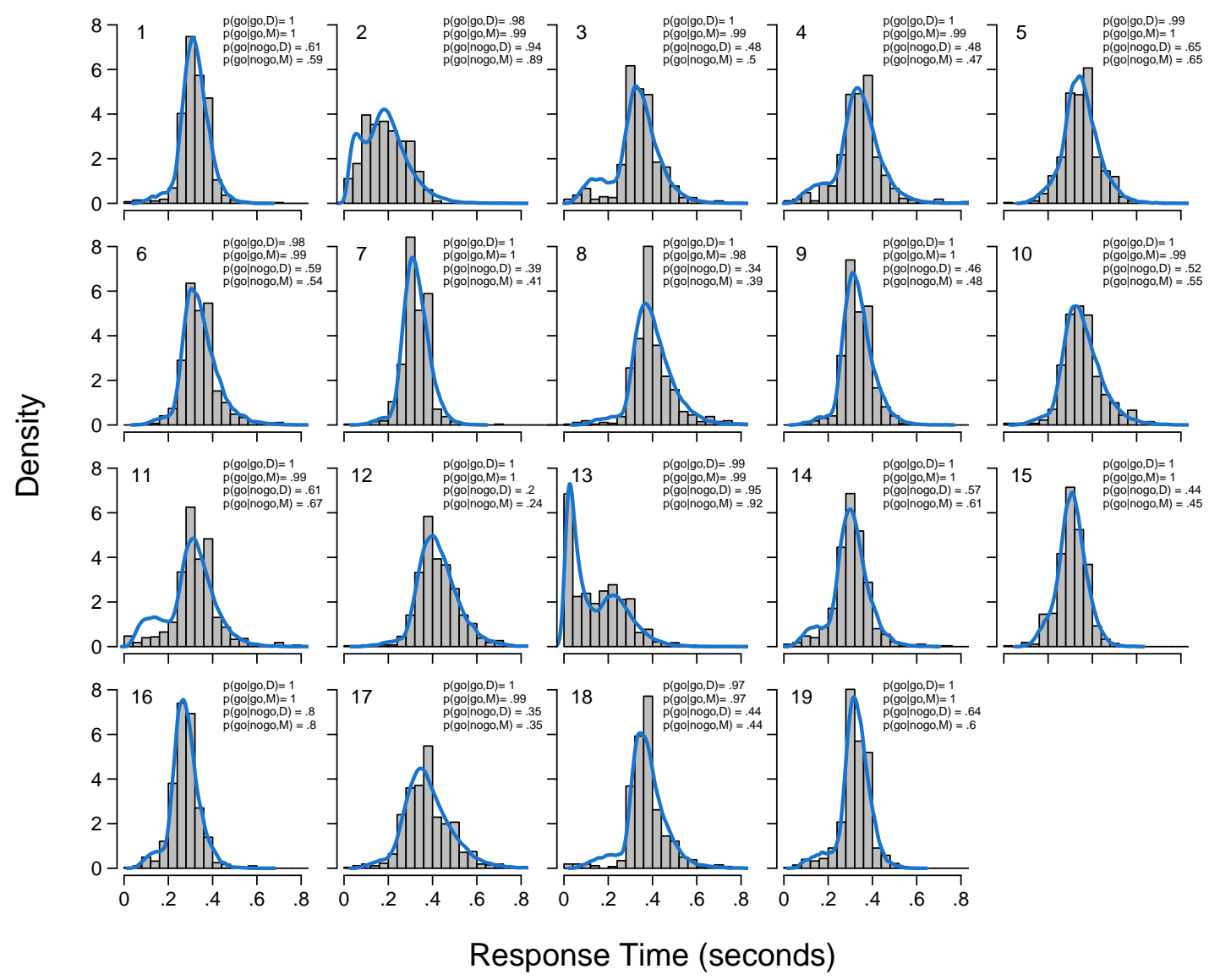

Figure 11. Individual participant (panels) RT distributions (gray histograms) with overlaid posterior predictive distribution of the TRDM (blue densities) from the go-nogo task in Experiment 1 of Hawkins et al. (2019), collapsing across correct and error RTs. The upper right of each panel shows the proportion of go responses on go and nogo trials observed in data for each participant, $p(g o \mid g o, D)$ and $p(g o \mid$ nogo, $D)$, respectively, and the same quantities as predicted by the TRDM, $p(g o \mid g o, M)$ and $p($ go $\mid$ nogo, $M)$. The upper left of each panel shows the participant ID. 
Table 11

TRDM parameter estimates and parameter recovery for the go-nogo task (Hawkins et al., 2019). Column 3 shows the posterior median of the population mean distribution transformed to the natural scale, with underset values showing $95 \%$ credible interval in parentheses. Column 4 shows parameter recovery in the same format as Table 3.

\begin{tabular}{|c|c|c|c|}
\hline Process & Parameter & $\begin{array}{l}\text { Posterior Median } \\
\quad(95 \% \mathrm{CI})\end{array}$ & $\begin{array}{l}\text { Parameter Recovery } \\
(95 \% \mathrm{CI})\end{array}$ \\
\hline \multirow{8}{*}{ Evidence } & Rate: go response to go stimuli & $\begin{array}{c}4.63 \\
(4.30,4.99)\end{array}$ & $\begin{array}{c}-.20 \\
(-1.47, .39)\end{array}$ \\
\hline & Rate: nogo response to go stimuli & 0 & - \\
\hline & Rate: go response to nogo stimuli & $\begin{array}{c}4.50 \\
(4.20,4.83)\end{array}$ & $\begin{array}{c}-.24 \\
(-1.44, .44)\end{array}$ \\
\hline & $\begin{array}{c}\text { Rate: nogo response to nogo stimuli } \\
\left(\gamma_{E, \text { nogo|nogo }}\right)\end{array}$ & $\begin{array}{c}4.47 \\
(3.80,5.24) \\
\end{array}$ & $\begin{array}{c}-.11 \\
(-1.42, .96) \\
\end{array}$ \\
\hline & $\begin{array}{c}\text { Threshold } \\
\left(\alpha_{E, \mu}\right)\end{array}$ & 1 & - \\
\hline & $\begin{array}{c}\text { Bias } \\
\left(\alpha_{E, \zeta}\right)\end{array}$ & 0 & - \\
\hline & $\begin{array}{c}\text { Variability } \\
\left(\sigma_{E}\right)\end{array}$ & $(.63, .82)$ & $\begin{array}{c}-.03 \\
-.28, .11)\end{array}$ \\
\hline & $\underset{\left(\tau_{E}\right)}{\operatorname{Non} \text {-decision time }}$ & $(.11, .16)$ & $\begin{array}{c}-.01 \\
(-.08, .02)\end{array}$ \\
\hline \multirow{4}{*}{ Timing } & $\begin{array}{c}\text { Rate } \\
\left(\gamma_{T}\right) \\
\end{array}$ & $\begin{array}{l}.10 \\
(.02, .72)\end{array}$ & $\begin{array}{c}.12 \\
(-.32,2.20) \\
\end{array}$ \\
\hline & $\underset{\left(\alpha_{T}\right)}{\text { Threshold }}$ & 1 & - \\
\hline & $\begin{array}{c}\text { Variability } \\
\left(\sigma_{T}\right)\end{array}$ & $\begin{array}{c}1.21 \\
(.98,1.50) \\
\end{array}$ & $\begin{array}{c}-.04 \\
(-.57, .09) \\
\end{array}$ \\
\hline & $\underset{\left(\tau_{T}\right)}{\text { Onset }}$ & 0 & - \\
\hline
\end{tabular}

no clear psychological interpretation in the present decision-making context. ${ }^{6}$

In contrast, the TRDM suggests that the unusual patterns in the go-nogo RT data are an outcome of a timing process that is more variable than the evidence process (i.e., $\sigma_{T}>$ $\sigma_{E}$; Table 11), even though the timing rate itself is quite low. This is a qualitatively different pattern to the four data sets examined in Part 1 of the paper, where the evidence process was consistently more variable than the timing process (i.e., $\sigma_{E}>\sigma_{T}$ ), and timing rates were generally higher. Yet the timing process still terminated a considerable proportion of trials on average: $21 \%$ [19, 22], aggregated across participants. However, in this context the aggregate is misleading. For example, one participant had $69 \%[66,71]$ of timer-predicted trials (participant 13 in Figure 11) and another had 43\% [38, 48] (participant 2). In contrast, other participants had fewer than $10 \%$ (e.g., participants 7, 9, and 12). These patterns make it clear that the TRDM explains the very fast responses observed in data as an outcome of

\footnotetext{
${ }^{6}$ The Weibull has an interpretation based on the asymptotic theory of extreme value statistics in terms of the fastest time to retrieve a large number of memory traces (Colonius, 1995). This has been used as a component of psychological theory in the context of the increasing number of traces accrued during skill acquisition (e.g., Logan, 1995) to provide a psychological motivation for the "Power Law of Practice" (but see Evans, Brown, Mewhort, \& Heathcote, 2018; Heathcote, Brown, \& Mewhort, 2000). However, this does not appear to provide a motivation for the Weibull in the context of timing.
} 
a rapid timing process.

The parameter estimates suggest a psychological interpretation that the precision of the timing process is difficult to maintain during highly monotonous tasks, as is the case with the SART, which was explicitly designed to induce mind wandering. Converging evidence is provided by the finding that participants with more variable timing processes tended to self-report greater rates of mind wandering during the task $\left(r=.60, B F_{10}=8.9\right) .{ }^{7}$ Participants with more variable evidence processes also tended to mind wander more often $\left(r=.61, B F_{10}=9.4\right)$; this pair of results is not likely to be attributable to a strong correlation between the variability parameters of the evidence and timing processes, which was only $r=.39\left(B F_{10}=1.4\right)$.

The associations between the two within-trial variability parameters of the TRDM and self-reported mind wandering are consistent with Hawkins et al. (2019). They found the strongest association between self-reported mind wandering and across-trial variability in drift rate of the RRM (based on the LBA architecture, $r=.66$ ), which is a remarkably similar result to the association we observed to within-trial variability in the evidence process $(r=.61)$. These results align with our earlier conclusion from the parameter recovery exercise in Part 2 of the paper: within-trial variability in the evidence process can absorb much of the effect that has previously been attributed to across-trial variability in drift rate. In support of this, there is a very strong correlation between across-trial variability in drift rate for the RRM (LBA architecture) and within-trial variability in the evidence process of the TRDM $\left(r=.80, B F_{10}=263\right)$.

\section{Individual Differences in Timing Precision}

In this final application we show that the timing process of the TRDM generalizes beyond decision making tasks, providing evidence that it is conceptually related to the representation of time. A core assumption of the TRDM is that timing is central to decision making. This implies that timing ability as measured in a timing-based task ought to relate to the timing process of the TRDM in a decision making task. Specifically, if the same sample of participants independently complete a choice task and an interval production task then we should observe an association between the parameters of the timing process of the TRDM in the choice task and the timing process run in isolation (without the evidence process) in the interval production task. The latter component is the standard application of the one-boundary diffusion process (inverse Gaussian distribution) to interval timing paradigms (e.g., Simen et al., 2011). We perform a targeted test of this hypothesis.

Method. We apply the TRDM to data from Experiment 2 of Miletić and Van Maanen (2019), where 64 participants each completed two tasks - a decision making task and an interval production task. Participants first completed a calibration phase of 250 trials of the decision making task. The task involved judging which of two circles 'flashed' more often, where a flash refers to displaying a circle and then quickly removing it. On each trial each circle had a fixed probability of flashing per display update, with the assignment of the target to the left or right circle randomized across trials. The target circle had a .7 probability of flashing and the distractor circle had a variable probability of flashing to

\footnotetext{
${ }^{7}$ Here and for all subsequent correlation analyses, we report non-parametric rank order correlation coefficients and corresponding Bayes Factors to circumvent potential violations of parametric assumptions. Details of the measurement of self-reported mind wandering are provided in Hawkins et al. (2019).
} 
manipulate choice difficulty $(.007, .21, .35, .49, .63)$, where the distractor probability was pseudo-randomly sampled on each trial such that there were 50 trials per difficulty level.

Performance in the calibration phase was used to identify participant-specific settings for the test blocks, including (1) probability of flashing for the distractor circle that led to approximately $80 \%$ correct responses and (2) the $75^{\text {th }}$ percentile of the participant's RT distribution. These tailored settings were used in two subsequent decision making blocks. There was a block with a 'short' deadline that used the participant-specific $75^{\text {th }}$ percentile from the calibration phase as an explicit response deadline. This block operated in much the same way as the explicit-deadline task we analyzed in Part 1 of the paper. There was also a 'long' deadline block that used a 5 s deadline. For both deadline blocks, if no response was given by the deadline the stimulus was removed and the participant received a penalty. Trials where participants gave a response after the deadline or failed to respond did not have a response recorded. Hence, in our modeling they were given a probability equal to the sum of the probabilities of making a decision for either outcome after the time of the deadline. For consistency with our earlier analyses, and given the general trends observed in Miletić and Van Maanen's (2019) data, we refer to the short deadline block as a 'speed emphasis' condition and the long deadline block as an 'accuracy emphasis' condition.

Participants also completed 200 trials of an interval production task. A trial in this task involved pressing a button to indicate the onset of an interval and pressing it again when the participant believed the required interval had ended. Participants were tasked with producing an interval that matched the $75^{\text {th }}$ percentile of their RT distribution from the calibration phase and received trialwise feedback on the accuracy of their interval production. For complete details we refer the reader to the primary source. One further detail that is relevant here is that Miletić and Van Maanen (2019) counterbalanced the order of the three test blocks across participants. For one group of participants the order was accuracy emphasis, then interval production and then speed emphasis, and for a second group the order was interval production, then speed emphasis and then accuracy emphasis. This ensured that the interval production task always directly preceded the speed emphasis condition, where the interval to produce was most relevant to decision performance. However, in exploratory analyses of the data we noticed that the second of these two orderings had an unintended effect: participants who completed the speed emphasis condition before the accuracy emphasis condition tended to show much smaller and sometimes no effect of the long deadline of the accuracy emphasis condition, because they appeared to carry over their speeded performance. This trend was clearly absent for the participants that completed the reverse ordering (accuracy before speed). For this reason, we restricted our analysis to the subset of 31 participants who completed the accuracy emphasis condition before the speed emphasis condition.

We estimated the TRDM based on the data from the choice and interval production tasks. The parameters of the choice task were estimated with the same assumptions as the information-controlled paradigms in Part 1 of the paper, and we estimated separate timing rate and precision parameters from the interval production task. This means parameters estimated from the choice task did not influence parameter estimates in the timing task, and vice versa. Appendix B has full details of the parameterization. All other modeling details were as described for the previous estimation exercises.

We used parameter estimates from the model fit to conduct a targeted test of our 
hypothesis that the timing process in the choice task is associated with the timing process in the interval production task. Specifically, we hypothesized that the precision of the timing process would be positively correlated across the two tasks (choice and interval production). We targeted the precision parameter as this is thought to index an individual difference trait, compared to the timer's rate parameter which is more task dependent (Simen et al., 2011, 2016).

Results. Figure 12 shows that the TRDM provides an excellent explanation of the data from both tasks: accuracy, correct and error RTs, and interval production RTs. Table 12 shows parameter estimates and once again demonstrates excellent parameter recovery. The TRDM mostly attributes the speed-accuracy tradeoff to a lower evidence threshold and a much faster timing rate under speed emphasis, lacking the non-decision time and mean rate components found for the data analyzed in Part 1 of the paper. Some differences are to be expected given that the choice task in this section is more like a combination of the two types of data sets we examined in Part 1: two conditions emphasized different elements of decision making (speed vs accuracy, like the information-controlled tasks) through the imposition of response deadlines (like the time-controlled tasks). Nevertheless, the TRDM still predicts a greater proportion of timer-terminated decisions when speed is emphasized $(36 \%[33,38])$ compared to accuracy $(19 \%[16,22])$, which is a reliable difference $(17 \%[13,20])$.

As in the results reported in Part 1, variability was lower for the timing accumulator than the evidence accumulators in the choice task. Variability was by far the lowest in the interval production task, suggesting that it was easier to achieve precise timing when that is the sole focus of the task. Most importantly, as hypothesized, the precision parameter of the timing process was positively correlated between the choice and interval production tasks $\left(r=.57, B F_{10}=43\right)$. This suggests that participants who made decisions with more precise timing processes were also more precise in the temporal intervals they produced. Importantly, these parameters were estimated independently: data from the choice task did not inform estimation of the parameters from the interval production task, and vice versa. That these results emerged from the structure in participants' data across two different tasks provides strong evidence for our proposition that the latent component of the TRDM we labeled as a timing process is indeed related to a latent ability to produce temporal intervals.

There was also a positive correlation between the precision parameter of the timing process in the interval production task and the precision of the evidence accumulation process in the choice task $\left(r=.56, B F_{10}=38\right)$. Taken together, the two positive associations suggest the presence of a trait indicating general processing variability, though this is speculative at this stage and requires further investigation. Nevertheless, consistent with this interpretation there was substantially less evidence for all other correlations between the precision of the timing process in the interval production task and the eight evidencerelated parameters of the choice task, all $.4<B F_{10}<10$. This provides evidence for divergent validity: the precision of temporal processing in the interval production task was not associated with non-precision related processing in the choice task.

\section{Conclusions}

We developed and tested a new model of dynamic decision making - the Timed Racing Diffusion Model (TRDM). The TRDM integrates theoretical accounts from tradi- 
Table 12

TRDM parameter estimates and parameter recovery for the choice and interal production tasks (Miletić \& Van Maanen, 2019). Estimates and recovered values are shown as a function of the instruction emphasis (speed, accuracy) where they were free to vary over this factor in the choice task. Column 4 shows the posterior median of the population mean distribution transformed to the natural scale, with underset values showing $95 \%$ credible interval in parentheses. Column 5 shows parameter recovery in the same format as Table 3.

\begin{tabular}{|c|c|c|c|c|}
\hline Process & Parameter & Emphasis & $\begin{array}{l}\text { Posterior Median } \\
(95 \% \mathrm{CI})\end{array}$ & $\begin{array}{c}\text { Parameter Recovery } \\
(95 \% \mathrm{CI})\end{array}$ \\
\hline \multirow{10}{*}{ Evidence } & \multirow{2}{*}{$\begin{array}{l}\text { Rate } \\
\left(\gamma_{E, \mu}\right)\end{array}$} & Speed & $\begin{array}{c}1.03 \\
(.76,1.38)\end{array}$ & $\begin{array}{c}-.07 \\
(-.60, .82)\end{array}$ \\
\hline & & Accuracy & $\begin{array}{c}1.10 \\
(.85,1.41)\end{array}$ & $\begin{array}{c}-.07 \\
(-.70, .68)\end{array}$ \\
\hline & \multirow{2}{*}{$\begin{array}{l}\text { Sensitivity } \\
\qquad\left(\gamma_{E, \zeta}\right)\end{array}$} & Speed & $\begin{array}{c}1.99 \\
(1.59,2.37)\end{array}$ & $\begin{array}{c}.06 \\
(-1.36, .90)\end{array}$ \\
\hline & & Accuracy & $\begin{array}{c}1.79 \\
(1.39,2.19)\end{array}$ & $\begin{array}{c}.09 \\
(-1.39, .76)\end{array}$ \\
\hline & \multirow[b]{2}{*}{$\begin{array}{l}\text { Threshold } \\
\qquad\left(\alpha_{E, \mu}\right)\end{array}$} & Speed & 1 & - \\
\hline & & Accuracy & $\begin{array}{c}1.61 \\
(1.42,1.83)\end{array}$ & $\begin{array}{c}0 \\
(-.60, .63) \\
\end{array}$ \\
\hline & $\begin{array}{c}\text { Bias } \\
\left(\alpha_{E, \zeta}\right) \\
\end{array}$ & - & $\begin{array}{c}.06 \\
(.02, .10)\end{array}$ & $\begin{array}{c}0 \\
(-.10, .08) \\
\end{array}$ \\
\hline & $\begin{array}{c}\text { Variability } \\
\left(\sigma_{E}\right)\end{array}$ & - & $\begin{array}{c}.94 \\
(.81,1.08) \\
\end{array}$ & $\begin{array}{c}.02 \\
(-.40, .20)\end{array}$ \\
\hline & \multirow{2}{*}{$\underset{\left(\tau_{E}\right)}{\text { Non-decision time }}$} & & $\begin{array}{c}.16 \\
(.13, .18)\end{array}$ & $\begin{array}{c}0 \\
(-.04, .11)\end{array}$ \\
\hline & & Accuracy & $\begin{array}{c}.10 \\
(.07, .15)\end{array}$ & $\begin{array}{c}.01 \\
(-.14, .10) \\
\end{array}$ \\
\hline \multirow{5}{*}{$\begin{array}{l}\text { Timing } \\
\text { (Choice) }\end{array}$} & \multirow{2}{*}{$\underset{\left(\gamma_{T}\right)}{\text { Rate }}$} & & $\begin{array}{c}1.31 \\
(1.10,1.55)\end{array}$ & $\begin{array}{c}.03 \\
(-.48, .61)\end{array}$ \\
\hline & & Accuracy & $\begin{array}{c}.24 \\
(.14, .40) \\
\end{array}$ & $\begin{array}{c}.08 \\
(-.57, .42)\end{array}$ \\
\hline & $\begin{array}{c}\text { Threshold } \\
\left(\alpha_{T}\right)\end{array}$ & - & 1 & - \\
\hline & $\begin{array}{c}\text { Variability } \\
\left(\sigma_{T}\right)\end{array}$ & - & $\begin{array}{c}.53 \\
(.43, .64) \\
\end{array}$ & $\begin{array}{c}-.01 \\
(-.19, .15)\end{array}$ \\
\hline & $\begin{array}{c}\text { Onset } \\
\left(\tau_{T}\right)\end{array}$ & - & .05 & - \\
\hline \multirow{4}{*}{$\begin{array}{c}\text { Timing } \\
\text { (Interval Production) }\end{array}$} & $\begin{array}{c}\text { Rate } \\
\left(\gamma_{T}\right)\end{array}$ & - & $\begin{array}{c}1.06 \\
(.90,1.25)\end{array}$ & $\begin{array}{c}0 \\
(-.04, .04) \\
\end{array}$ \\
\hline & $\begin{array}{c}\text { Threshold } \\
\left(\alpha_{T}\right)\end{array}$ & - & 1 & - \\
\hline & $\begin{array}{c}\text { Variability } \\
\left(\sigma_{T}\right)\end{array}$ & - & $\begin{array}{c}.14 \\
(.12, .17)\end{array}$ & $\begin{array}{c}0 \\
(-.02, .02) \\
\end{array}$ \\
\hline & $\underset{\left(\tau_{T}\right)}{\text { Onset }}$ & - & .05 & - \\
\hline
\end{tabular}




\section{Choice Task}
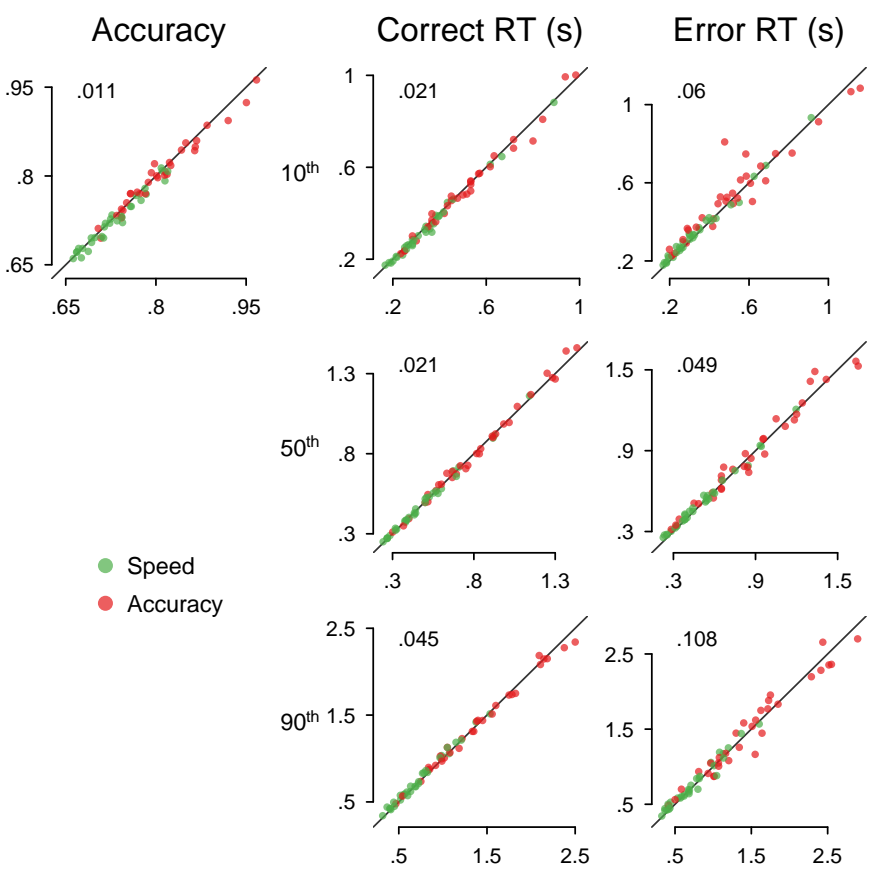

\section{Timing Task}
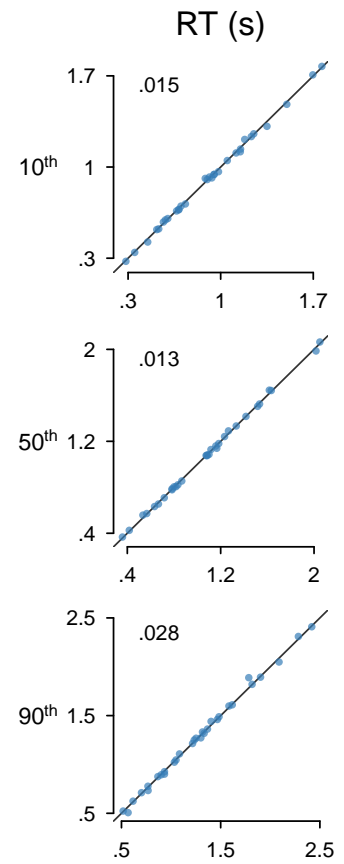

Figure 12. Observed ( $x$-axes) vs. predicted ( $y$-axes) data for individual participants in the choice and interval production tasks of Experiment 2 in Miletić and Van Maanen (2019). The leftmost three columns represent the choice task (accuracy, correct RTs, error RTs) and the rightmost column represents the interval production task (RTs). For RT data, the rows show the $10^{t h}, 50^{t h}$ and $90^{t h}$ percentiles of RT distribution. Each dot represents a single participant. The value in the upper left of each panel shows the root mean square error.

tionally disparate fields of decision making (evidence accumulation) and time representation (temporal accumulation). It does this in a unified architecture with surprisingly simple yet theoretically and neurally motivated distributional forms: one-boundary diffusion processes, for both evidence and timing accumulation processes. This simplicity is one of the primary strengths of the TRDM.

We provided evidence for the descriptive adequacy of the TRDM and its ability to provide theoretically coherent parametric explanation of decision making in a range of contexts. This included information-controlled phenomena, like the speed-accuracy tradeoff, and time-controlled phenomena, like decisions in the presence of internally or externally imposed response deadlines. These are major phenomena typically considered to be the 'benchmarks' new models must meet to be competitive in the field. To our knowledge, the TRDM is the only model that provides a complete explanation of performance in the informationand time-controlled paradigms without any modification to its architecture. Recent debates in the literature have suggested that dynamic decision models with collapsing thresholds or urgency signals are essential to ensure decisions are made before response deadlines (timecontrolled phenomena), however these assumptions are not typically required for decisions 
in the absence of deadlines (information-controlled phenomena), and sometimes hurt performance. We showed that the TRDM - with identical structural assumptions - explains performance in information- and time-controlled paradigms. Furthermore, and unlike many modern dynamic decision models, the TRDM is a complete measurement model in the sense that parameter recovery was excellent for all parameterizations in all of the designs we examined (cf. Tables 3, 4, 6, 7, 11, and 12).

We departed from recent literature in simplifying the architecture of the TRDM, eschewing the standard assumption of across-trial variability in parameters. We do not argue that there is never variability across trials, and indeed it remains possible that in some tasks it is an important component. In such cases it may be useful to combine a timing accumulator with a choice model that assumes across-trial variability, such as the LBA, as it is likely that this combination would inherit many of the advantages afforded by the TRDM. That said, our findings with the TRDM do demonstrate that across-trial variability does not need to be included in order to explain the relative speed of correct and error responses. Not having to necessarily include across-trial variability makes the TRDM theoretically and computationally simpler than its competitors, and avoids the need to make assumptions about the distributional form of across-trial variability that can be hard to test (Jones \& Dzhafarov, 2014). Theoretically, the shift in the explanatory role typically assigned to across-trial variability to the relative speed and precision of the timing process opens the door to novel investigations into contextual factors affecting the timing process; for example, does mind wandering cause timing to be more variable, and how do response deadlines modulate temporal processing? Computationally, the TRDM is a closed-form combination of the well-understood one-boundary diffusion process (i.e., shifted Wald, inverse Gaussian distribution). This makes computation of the TRDM's density and distribution functions extremely rapid; in this regard it is on par with the currently most tractable yet complete model of decision making, the LBA. We hope this ease of use will increase uptake of the TRDM. To that end, code for applying the TRDM to all data sets considered in this paper is available at osf.io/5cf $7 \mathrm{w}$.

Despite the TRDM's simplifying assumptions, it has an explanatory scope that goes beyond standard choice tasks. This imbues it with greater generality than standard evidence accumulation models of decision making, opening new avenues for investigation. We provided preliminary evidence for this in two domains. First, the TRDM is a domaingeneral choice model that can serve as a drop-in replacement for a domain-specific model of pre-emptive responding, with minimal loss in goodness of fit. The TRDM also provided theoretically consistent associations with participants' self-reported mind wandering. Although not informed by the self-report data it produced inferences consistent with contemporaneous literature on mind wandering. The TRDM also provided the first unified explanation of performance across exemplar tasks from two literatures that have traditionally been studied in isolation: decision making and interval production. The TRDM provided an excellent explanation of performance in both tasks, and demonstrated a strong association between individual timing precision differences in decision making and temporal interval production tasks. This supports the validity of our assumption that subjective timing is a central component to decision making and opens up new research avenues between how we make decisions and how we perceive the passing of time. 


\section{Future Directions}

While we tested some TRDM assumptions in this paper, the model will benefit from additional targeted empirical tests. For instance, in all applications we assumed that timingbased responses were pure guesses. That is, for a choice between $N$ options the response probabilities for timer responses were $\frac{1}{N}$, implying that timer responses are not informed by the state of the evidence process at the time of response. This was sufficient in the scenarios we studied, though it is likely to be insufficient in others. Consider a brightness discrimination task where the participant is to select the brightest stimulus from $N=3$ options, where two options are bright and one is dark. ${ }^{8}$ Given its similarity to the bright target, the bright distractor may be more likely to be erroneously chosen than the dark distractor such that the distribution of errors is not uniform over options. For timerbased errors, the TRDM as presented here predicts both distractors will be chosen with probability $\frac{1}{N}$. Fitting such data may, therefore, require the TRDM to permit access to partial information, or some other biased guessing process.

Although our focus has been at the level of pure guessing vs. partial information, the ideas behind these error models have also been investigated beyond the speeded decision making context we studied here. As a case in point, Loomes, Moffatt, and Sugden (2002) raised parallel questions in the context of risky choice. They implemented different error models in an expected utility framework for choice between risky prospects. Two of those error models are essentially static (i.e., constant over time) versions of the partial information and pure-guessing TRDMs discussed here. Respectively, their Fechner model assumed randomness in the comparison of the risky prospect to latent (true) preferences, like a noisy evaluation of stimulus evidence, and their constant error or tremble model assumed randomness at the stage of action, like a lapse-based response that does not consider stimulus information. A key insight that can be exploited in future tests is that tremble models, like the pure-guessing version of the TRDM, are insensitive to the properties of distractors. This makes the various error models difficult to discriminate in the two-alternative choice tasks we considered here though they will be of key interest in multi-choice applications of the TRDM.

Beyond additional assumption tests, the TRDM has considerable scope that will be exciting to explore in future research. One potential application is don't know responding, where participants have three responses: two definitive choices about the stimulus and a don't know response. Don't-know responses give the decision maker a way to 'opt out' of the choice. The TRDM's timing process is essentially a don't-know signal: if the decision process times out then the decision maker has concluded that insufficient evidence was evaluated in the time frame they were willing to commit to the decision. Although 'don't know' is a common outcome of every day decisions, don't-know responding has no natural implementation in most models of two-alternative forced choice decisions (but see Reynolds et al., in press, for a mechanism based on the relative evidence for the definitive choices).

The timing process can even be considered in a completely non-timed manner - as a reference level in absolute judgment. Consider a line-up judgment where the decision maker is tasked with identifying a perpetrator (the target) from other candidate suspects (distractors), or identifying that the perpetrator is absent. This is an absolute judgment: the

\footnotetext{
${ }^{8}$ We thank an anonymous reviewer for this suggested experiment.
} 
decision maker must collect enough information to be sufficiently confident the perpetrator is present. In this context, the input signal to the timing process serves as the reference level of evidence. To illustrate with a toy example, let's say the perpetrator has evidence strength 5 and a distractor has evidence strength 1 , and the judgment criterion is 3 . When the true perpetrator is presented, the accumulator for a 'positive' response is driven by a rate of $5-3=2$, the accumulator for a 'negative' response is driven by $3-5=-2$, and the 'opt out' accumulator is driven by a constant rate of, say, 1. This allows the opt-out accumulator to censor the slow 'positive' responses, which are themselves the most likely to be uncertain (i.e., lower signal strength, on average). The principle here is that the TRDM allows us to consider situations where the decision maker sets a reference level of evidence that they wish to exceed before taking action based on the evidence. This contrasts with conventional evidence accumulation models where given enough time an evidence threshold associated with choosing a suspect is guaranteed to be exceeded if there is at least one positive accumulation rate.

We leave it to future research to investigate these prospects. Our hope is that models from different literatures - like speeded decision making and risky choice - and different types of decision outcomes - like forced choice, don't know and opting out - can be unified when viewed through the lens of the TRDM framework. This form of linking across decision making and timing literatures was a major motivation for the TRDM's development, and it is our hope that such links can be extended further in the future. 


\section{References}

Audley, R. (1960). A stochastic model for individual choice behavior. Psychological Review, 67, $1-15$.

Balci, F., \& Simen, P. (2014). Decision processes in temporal discrimination. Acta Psychologica, 149, 157-168.

Balci, F., \& Simen, P. (2016). A decision model of timing. Current Opinion in Behavioral Sciences, 8, 94-101.

Bhatia, S., \& Mullett, T. L. (2016). The dynamics of deferred decision. Cognitive Psychology, 86, $112-151$.

Boag, R. J., Strickland, L., Loft, S., \& Heathcote, A. (2019). Strategic attention and decision control support prospective memory in a complex dual-task environment. Cognition, 191, 103974.

Bowman, N. E., Kording, K. P., \& Gottfried, J. A. (2012). Temporal integration of olfactory perceptual evidence in human orbitofrontal cortex. Neuron, 75, 916-927.

Brooks, S. P., \& Gelman, A. (1998). General methods for monitoring convergence of iterative simulations. Journal of Computational and Graphical Statistics, 7, 434-455.

Brown, S. D., \& Heathcote, A. (2005). A ballistic model of choice response time. Psychological Review, 112, 117-128.

Brown, S. D., \& Heathcote, A. (2008). The simplest complete model of choice response time: Linear ballistic accumulation. Cognitive psychology, 57(3), 153-178.

Churchland, A. K., Kiani, R., \& Shadlen, M. N. (2008). Decision-making with multiple alternatives. Nature Neuroscience, 11, 693-702.

Cisek, P., Puskas, G. A., \& El-Murr, S. (2009). Decisions in changing conditions: the urgency-gating model. The Journal of Neuroscience, 29(37), 11560-11571.

Colonius, H. (1995). The instance theory of automaticity: Why the Weibull? Psychological Review, 102(4), 744.

Coltheart, M., Rastle, K., Perry, C., Langdon, R., \& Ziegler, J. (2001). DRC: A dual route cascaded model of visual word recognition and reading aloud. Psychological Review, 108, 204-ñ256.

Diller, D. E., Nobel, P. A., \& Shiffrin, R. M. (2001). An arc-rem model for accuracy and response time in recognition and recall. Journal of Experimental Psychology: Learning, Memory, and Cognition, $27(2), 414-435$.

Ditterich, J. (2006). Evidence for time-variant decision making. European Journal of Neuroscience, $24(12), 3628-3641$.

Donkin, C., Brown, S. D., \& Heathcote, A. (2009). The overconstraint of response time models: Rethinking the scaling problem. Psychonomic Bulletin \& Review, 16(6), 1129-1135.

Drugowitsch, J., Moreno-Bote, R., Churchland, A. K., Shadlen, M. N., \& Pouget, A. (2012). The cost of accumulating evidence in perceptual decision making. Journal of Neuroscience, 32, $3612-3628$.

Dutilh, G., Annis, J., Brown, S. D., Cassey, P., Evans, N. J., Grasman, R. P., ... Donkin, C. (2018). The quality of response time data inference: A blinded, collaborative assessment of the validity of cognitive models. Psychonomic Bulletin $\&$ Review.

Evans, N. J., Brown, S. D., Mewhort, D. J. K., \& Heathcote, A. (2018). Refining the law of practice. Psychological Review, 125(4), 592-605.

Evans, N. J., \& Hawkins, G. E. (2019). When humans behave like monkeys: Feedback delays and extensive practice increase the efficiency of speeded decisions. Cognition, 184, 11-18.

Evans, N. J., Hawkins, G. E., \& Brown, S. D. (2019). The role of passing time in decision-making. Journal of Experimental Psychology: Learning, Memory, and Cognition.

Evans, N. J., Trueblood, J. S., \& Holmes, W. R. (2019). A parameter recovery assessment of time-variant models of decision-making. Behavior Research Methods, 1-14.

Forstmann, B. U., Dutilh, G., Brown, S., Neumann, J., Von Cramon, D. Y., Ridderinkhof, K. R., \& Wagenmakers, E.-J. (2008). Striatum and pre-sma facilitate decision-making under time 
pressure. Proceedings of the National Academy of Sciences, 105 (45), 17538-17542.

Frazier, P. I., \& Yu, A. J. (2008). Sequential hypothesis testing under stochastic deadlines. Advances in Neural Information Processing Systems 20.

Gao, J., Tortell, R., \& McClelland, J. L. (2011). Dynamic integration of reward and stimulus information in perceptual decision-making. PloS one, $6(3)$.

Garton, R., Reynolds, A., Hinder, M. R., \& Heathcote, A. (2019). Equally flexible and optimal response bias in older compared to younger adults. Psychology and Aging, 34(6), 821-835.

Grainger, J., \& Jacobs, A. M. (1996). Orthographic processing in visual word recognition: A multiple read-out model. Psychological Review, 103, 518-565.

Grondin, S. (2010). Timing and time perception: A review of recent behavioral and neuroscience findings and theoretical directions. Attention, Perception, \&6 Psychophysics, 72 (3), 561-582.

Hawkins, G. E., Forstmann, B. U., Wagenmakers, E.-J., Ratcliff, R., \& Brown, S. D. (2015). Revisiting the evidence for collapsing boundaries and urgency signals in perceptual decisionmaking. Journal of Neuroscience, 35, 2476-2484.

Hawkins, G. E., Mittner, M., Forstmann, B. U., \& Heathcote, A. (2017). On the efficiency of neurally-informed cognitive models to identify latent cognitive states. Journal of Mathematical Psychology, 76, 142-155.

Hawkins, G. E., Mittner, M., Forstmann, B. U., \& Heathcote, A. (2019). Modeling distracted performance. Cognitive psychology, 112, 48-80.

Hawkins, G. E., Wagenmakers, E.-J., Ratcliff, R., \& Brown, S. D. (2015). Discriminating evidence accumulation from urgency signals in speeded decision making. Journal of Neurophysiology, $114,40-47$.

Heathcote, A. (2004). Fitting Wald and ex-Wald distributions to response time data: An example using functions for the S-PLUS package. Behavior Research Methods, Instruments, \& Computers, 36, 678-694.

Heathcote, A., Brown, S., \& Mewhort, D. J. K. (2000). The power law repealed: The case for an exponential law of practice. Psychonomic Bulletin 85 Review, 7, 185-207.

Heathcote, A., Brown, S. D., \& Wagenmakers, E.-J. (2015). An introduction to good practices in cognitive modeling. In B. U. Forstmann \& E.-J. Wagenmakers (Eds.), An introduction to model-based cognitive neuroscience. New York: Springer.

Heathcote, A., Holloway, E., \& Sauer, J. (2019). Confidence and varieties of bias. Journal of Mathematical Psychology, 90, 31-46.

Heathcote, A., Lin, Y.-S., Reynolds, A., Strickland, L., Gretton, M., \& Matzke, D. (2019). Dynamic models of choice. Behavior Research Methods, 51(2), 961-985.

Heathcote, A., \& Love, J. (2012). Linear deterministic accumulator models of simple choice. Frontiers in Psychology, 3, n/a. doi: 10.3389/fpsyg.2012.00292.

Heathcote, A., Wagenmakers, E.-J., \& Brown, S. D. (2014). The falsifiability of actual decisionmaking models.

Heitz, R. P. (2014). The speed-accuracy tradeoff: History, physiology, methodology, behavior. Frontiers in Neuroscience, 8, n/a. doi: 10.3389/fpsyg.2014.00150.

Jones, M., \& Dzhafarov, E. N. (2014). Unfalsifiability and mutual translatability of major modelling schemes for choice reaction time. Psychological Review, 121, 1-32.

Katsimpokis, D., Hawkins, G. E., \& Van Maanen, L. (in press). Not all speed-accuracy tradeoff manipulations have the same psychological effect. Computational Brain $\&$ Behavior.

Laming, D. R. J. (1968). Information theory of choice-reaction times. London: Academic Press.

Logan, G. D. (1995). The Weibull distribution, the power law, and the instance theory of automaticity. Psychological Review, 102, 751-756.

Logan, G. D., Van Zandt, T., Verbruggen, F., \& Wagenmakers, E.-J. (2014). On the ability to inhibit thought and action: General and special theories of an act of control. Psychological Review, 121, 66-95.

Loomes, G., Moffatt, P. G., \& Sugden, R. (2002). A microeconometric test of alternative stochastic 
theories of risky choice. Journal of Risk and Uncertainty, 24(2), 103-130.

Matzke, D., \& Wagenmakers, E.-J. (2009). Psychological interpretation of the ex-Gaussian and shifted Wald parameters: A diffusion model analysis. Psychonomic Bulletin E Review, 16, $798-817$.

McElree, B. (2001). Working memory and focal attention. Journal of Experimental Psychology: Learning, Memory, and Cognition, 27(3), 817.

McElree, B., \& Dosher, B. A. (1989). Serial position and set size in short-term memory: the time course of recognition. Journal of Experimental Psychology: General, $118(4), 346$.

Merchant, H., Harrington, D. L., \& Meck, W. H. (2013). Neural basis of the perception and estimation of time. Annual Review of Neuroscience, 36, 313-336.

Miletić, S., \& Van Maanen, L. (2019). Caution in decision-making under time pressure is mediated by timing ability. Cognitive Psychology, 110, 16-29.

Milosavljevic, M., Malmaud, J., Huth, A., Koch, C., \& Rangel, A. (2010). The drift diffusion model can account for the accuracy and reactime of value-based choices under high and low time pressure. Judgment and Decision Making, 5, 437-449.

Mulder, M. J., Wagenmakers, E.-J., Ratcliff, R., Boekel, W., \& Forstmann, B. U. (2012). Bias in the brain: A diffusion model analysis of prior probability and potential payoff. Journal of Neuroscience, 32, 2335-2343.

Nobel, P. A., \& Shiffrin, R. M. (2001). Retrieval processes in recognition and cued recall. Journal of Experimental Psychology: Learning, Memory, and Cognition, 27(2), 384-413.

Noorbaloochi, S., Sharon, D., \& McClelland, J. L. (2015). Payoff information biases a fast guess process in perceptual decision making under deadline pressure: Evidence from behavior, evoked potentials, and quantitative model comparison. Journal of Neuroscience, 35(31), 1098911011.

Nosofsky, R. M., Little, D., Donkin, C., \& Fific, M. (2011). Short-term memory scanning viewed as exemplar-based categorization. Psychological Review, 118, 280-315.

Nosofsky, R. M., \& Palmeri, T. J. (1997). An exemplar-based random walk model of speeded classification. Psychological Review, 104, 266-300.

O’Connell, R. G., Shadlen, M. N., Wong-Lin, K., \& Kelly, S. P. (2018). Bridging neural and computational viewpoints on perceptual decision-making. Trends in Neurosciences, 41(11), 838-852.

Ollman, R. T., \& Billington, M. J. (1972). The deadline model for simple reaction times. Cognitive Psychology, 3(2), 311-336.

Palestro, J. J., Weichart, E., Sederberg, P. B., \& Turner, B. M. (2018). Some task demands induce collapsing bounds: Evidence from a behavioral analysis. Psychonomic Bulletin 6 Review, 25, 1225-1248.

Rae, B., Heathcote, A., Donkin, C., Averell, L., \& Brown, S. (2014). The hare and the tortoise: Emphasizing speed can change the evidence used to make decisions. Journal of Experimental Psychology: Learning, Memory, and Cognition, 40(5), 1226.

Ratcliff, R. (1978). A theory of memory retrieval. Psychological Review, 85, 59-108.

Ratcliff, R. (1980). A note on modeling accumulation of information when the rate of accumulation changes over time. Journal of Mathematical Psychology.

Ratcliff, R. (1988). Continuous versus discrete information processing: Modeling the accumulation of partial information. Psychological Review, 95, 238-255.

Ratcliff, R. (2002). A diffusion model account of response time and accuracy in a brightness discrimination task: Fitting real data and failing to fit fake but plausible data. Psychonomic Bulletin \& Review, 9, 278-291.

Ratcliff, R. (2006). Modeling response signal and response time data. Cognitive Psychology, 53, $195 \tilde{n}-237$.

Ratcliff, R., Gomez, P., \& McKoon, G. (2004). Diffusion model account of lexical decision. Psychological Review, 111, 159-182. 
Ratcliff, R., \& McKoon, G. (2008). The diffusion decision model: Theory and data for two-choice decision tasks. Neural Computation, 20, 873-922.

Ratcliff, R., \& Rouder, J. N. (1998). Modeling response times for two-choice decisions. Psychological Science, 9(5), 347-356.

Ratcliff, R., Smith, P. L., Brown, S. D., \& McKoon, G. (2016). Diffusion decision model: Current issues and history. Trends in Cognitive Sciences, 20, 260-281.

Ratcliff, R., \& Tuerlinckx, F. (2002). Estimating parameters of the diffusion model: Approaches to dealing with contaminant reaction times and parameter variability. Psychonomic Bulletin $\&$ Review, 9, 438-481.

Ratcliff, R., Van Zandt, T., \& McKoon, G. (1999). Connectionist and diffusion models of reaction time. Psychological Review, 102, 261-300.

Ratcliff, R., Voskuilen, C., \& Teodorescu, A. (2018). Modeling 2-alternative forced-choice tasks: Accounting for both magnitude and difference effects. Cognitive Psychology, 103, 1-22.

Reynolds, A., Garton, R., Kvam, P., Griffin, V., Sauer, J., Osth, A., \& Heathcote, A. (in press). A dynamic model of deciding not to choose. Journal of Experimental Psychology: General.

Ridderinkhof, R. (2002). Micro- and macro-adjustments of task set: Activation and suppression in conflict tasks. Psychological Research, 66, 312-323.

Robertson, I. H., Manly, T., Andrade, J., Baddeley, B. T., \& Yiend, J. (1997). 'Oops!': Performance correlates of everyday attentional failures in traumatic brain injured and normal subjects. Neuropsychologia, 35, 747-758.

Roitman, J. D., \& Shadlen, M. N. (2002). Response of neurons in the lateral intraparietal area during a combined visual discrimination reaction time task. The Journal of Neuroscience, 22(21), 9475-9489.

Shadlen, M. N., \& Kiani, R. (2013). Decision making as a window on cognition. Neuron, 80, 791-806.

Shi, Z., Allenmark, F., Zhu, X., Elliott, M. A., \& Müller, H. J. (2020). To quit or not to quit in dynamic search. Attention, Perception, \& Psychophysics, 82, 799-817.

Simen, P., Balci, F., deSouza, L., Cohen, J. D., \& Holmes, P. (2011). A model of interval timing by neural integration. The Journal of Neuroscience, 31, 9238-9253.

Simen, P., Contreras, D., Buck, C., Hu, P., Holmes, P., \& Cohen, J. D. (2009). Reward rate optimization in two-alternative decision making: Empirical tests of theoretical predictions. Journal of Experimental Psychology: Human Perception and Performance, 35, 1865-1897.

Simen, P., Rivest, F., Ludvig, E. A., Balci, F., \& Killeen, P. (2013). Timescale invariance in the pacemaker-accumulator family of timing models. Timing \& Time Perception, 1(2), 159-188.

Simen, P., Vlasov, K., \& Papadakis, S. (2016). Scale (in)variance in a unified diffusion model of decision making and timing. Psychological Review, 123, 151-181.

Singmann, H., Brown, S., Gretton, M., Heathcote, A., Voss, A., Voss, J., \& Terry, A. (2019). rtdists: Response time distributions [Computer software manual]. ( $\mathrm{R}$ package version $0.10-0$ )

Smallwood, J., Davies, J. B., Heim, D., Finnigan, F., Sudberry, M., O'Connor, R., \& Obonsawin, M. (2004). Subjective experience and the attentional lapse: Task engagement and disengagement during sustained attention. Consciousness and Cognition, 13, 657-690.

Smallwood, J., \& Schooler, J. W. (2006). The restless mind. Psychological Bulletin, 132, 946-958.

Smith, P. L. (2000). Stochastic dynamic models of response time and accuracy: A foundational primer. Journal of Mathematical Psychology, 44, 408-463.

Smith, P. L. (2010). From poisson shot noise to the integrated Ornstein-Uhlenbeck process: Neurally principled models of information accumulation in decision-making and response time. Journal of Mathematical Psychology, 54 (2), 266-283.

Smith, P. L., Ratcliff, R., \& McKoon, G. (2014). The diffusion model is not a deterministic growth model: Comment on jones and dzhafarov (2014).

Spiegelhalter, D. J., Best, N. G., Carlin, B. P., \& van der Linde, A. (2002). Bayesian measures of model complexity and fit. Journal of Royal Statistician Society Series B, 64(4), 583-639. 
Spieser, L., Servant, M., Hasbroucq, T., \& Burle, B. (2017). Beyond decision! Motor contribution to speed-accuracy trade-off in decision-making. Psychonomic Bulletin \& Review, 24(3), 950956.

Starns, J. J., Pazzaglia, A. M., Rotello, C. M., Hautus, M. J., \& Macmillan, N. A. (2013). Unequalstrength source zROC slopes reflect criteria placement and not (necessarily) memory processes. Journal of Experimental Psychology: Learning, Memory, and Cognition, 39(5), 1377-1392.

Stone, M. (1960). Models for choice-reaction time. Psychometrika, 25, 251-260.

Strickland, L., Loft, S., Remington, R. W., \& Heathcote, A. (2018). Racing to remember: A theory of decision control in event-based prospective memory. Psychological Review, 125(6), 851-887.

Tajima, S., Drugowitsch, J., \& Pouget, A. (2016). Optimal policy for value-based decision-making. Nature Communications, 7, 12400.

Tillman, G., Van Zandt, T., \& Logan, G. D. (in press). Sequential sampling models without random between-trial variability: The racing diffusion model of speeded decision making. Psychonomic Bulletin 8 Review.

Turner, B. M. (2019). Toward a common representational framework for adaptation. Psychological Review, $126(5), 660$.

Turner, B. M., \& Sederberg, P. B. (2014). A generalized, likelihood-free method for posterior estimation. Psychonomic Bulletin \& Review, 21(2), 227-250.

Turner, B. M., Sederberg, P. B., Brown, S. D., \& Steyvers, M. (2013). A method for efficiently sampling from distributions with correlated dimensions. Psychological Methods, 18, 368-384.

Turner, B. M., Sederberg, P. B., \& McClelland, J. L. (2016). Bayesian analysis of simulation-based models. Journal of Mathematical Psychology, 72, 191-199.

Usher, M., \& McClelland, J. L. (2001). The time course of perceptual choice: the leaky, competing accumulator model. Psychological review, 108(3), 550-592.

Van Zandt, T., Colonius, H., \& Proctor, R. W. (2000). A comparison of two response time models applied to perceptual matching. Psychonomic Bulletin \& Review, 7, 208-256.

Voskuilen, C., Ratcliff, R., \& Smith, P. L. (2016). Comparing fixed and collapsing boundary versions of the diffusion model. Journal of Mathematical Psychology, 73, 59-79.

Wagenmakers, E.-J., \& Farrell, S. (2004). AIC model selection using Akaike weights. Psychonomic Bulletin \& Review, 11, 192-196.

Wagenmakers, E.-J., Ratcliff, R., Gomez, P., \& McKoon, G. (2008). A diffusion model account of criterion shifts in the lexical decision task. Journal of Memory and Language, 58 (1), 140-159.

White, C. N., \& Poldrack, R. A. (2014). Decomposing bias in different types of simple decisions. Journal of Experimental Psychology: Learning, Memory, and Cognition, 40(2), 385.

Wolfe, J. M., \& Van Wert, M. J. (2010). Varying target prevalence reveals two dissociable decision criteria in visual search. Current Biology, 20(2), 121-124. 
Appendix A

Hierarchical Bayesian Parameter Estimation

We used a hierarchical Bayesian framework to simultaneously estimate participant and group-level parameters for all of the decision making models discussed in the main text (TRDM, RDM, LBA, DDM). Parameters were estimated by differential evolution Markov chain Monte Carlo (DE-MCMC) sampling, using the default settings unless noted otherwise (Turner, Sederberg, Brown, \& Steyvers, 2013). We took 8,000 posterior samples from each of $3 k$ MCMC chains, where $k$ was the number of model parameters at the participant (i.e., data) level and varied as a function of study design, with a burnin period of 6,000 samples. During the first 2,000 samples of the burnin period we conducted a "migration" update step for participant-level parameters with probability .05, which helps to remove chains stuck in low likelihood regions of the parameter space (for details, see Turner et al., 2013). We kept every second sample from the final set of 2,000 posterior samples for a total of 1,000k samples from the posterior distribution of the parameters. Convergence was monitored through visual inspection and the multivariate potential scale reduction factor $(\widehat{R}$; Brooks \& Gelman, 1998).

For all models, parameters $\theta_{i}$ for participant $i$, where $i \in\{1,2, \ldots, S\}$ and $S$ is the number of participants in each data set, were transformed to the real line and drawn hierarchically from Normally-distributed group-level hyper distributions with mean $\theta^{\mu}$ and standard deviation $\theta^{\sigma}$, for $\theta=\left(\theta_{1}, \ldots, \theta_{k}\right)$. We assumed mildly informative prior distributions for the group-level parameters, which allowed the estimated parameters to vary across a range of plausible values.

\section{Timed Racing Diffusion Model (TRDM)}

The TRDM has 10 parameters in total, as described in the main text. However, we did not freely estimate the onset time or the threshold of the timing process in any data set, instead fixing them to constants $\left(\tau_{T}=.05 \mathrm{~s}, \alpha_{T}=1\right)$. We also set the mean evidence threshold to a fixed value as the scaling parameter $\left(\alpha_{E, \mu}=1\right)$; we only estimated a mean evidence threshold when the study design required more than one evidence threshold across conditions (e.g., speed vs accuracy emphasis conditions). Together this reduced the TRDM to 7 base parameters to estimate from data. The parameter vector for participant $i$ was 
$\theta_{i} \in\left\{\gamma_{E, \mu}, \gamma_{E, \zeta}, \alpha_{E, \mu}, \alpha_{E, \zeta}, \sigma_{E}, \tau_{E}, \gamma_{T}, \sigma_{T}\right\}$. Formally, the TRDM was defined as:

Data level :

$\left(R T_{i}\right.$, response $\left._{i}\right) \sim \operatorname{TRDM}\left(\theta_{i}\right)$

Group level :

$$
\begin{aligned}
\log \left(\theta_{i}\right) & \sim N\left(\theta^{\mu}, \theta^{\sigma}\right), \quad \forall \theta_{i} \in\left\{\theta_{i}\right\} \backslash\left\{\gamma_{E, \zeta, i}, \alpha_{E, \zeta, i}\right\} \\
\gamma_{E, \zeta, i} & \sim N\left(\gamma_{E, \zeta}^{\mu}, \gamma_{E, \zeta}^{\sigma}\right) \\
\alpha_{E, \zeta, i} & \sim N\left(\alpha_{E, \zeta}^{\mu}, \alpha_{E, \zeta}^{\sigma}\right)
\end{aligned}
$$

Prior distributions :

$$
\begin{aligned}
\gamma_{E, \mu}^{\mu} & \sim N(.5,1) \\
\gamma_{E, \zeta}^{\mu}, \gamma_{T}^{\mu} & \sim N(1,1) \\
\alpha_{E, \mu}^{\mu}, \alpha_{E, \zeta}^{\mu}, \tau_{E}^{\mu} & \sim N(0,1) \\
\sigma_{E}^{\mu}, \sigma_{T}^{\mu} & \sim N(-.5,1) \\
\gamma_{E, \mu}^{\sigma}, \gamma_{E, \zeta}^{\sigma}, \alpha_{E, \mu}^{\sigma}, \alpha_{E, \zeta}^{\sigma}, \sigma_{E}^{\sigma}, \tau_{E}^{\sigma}, \gamma_{T}^{\sigma}, \sigma_{T}^{\sigma} & \sim \Gamma(1,1)
\end{aligned}
$$

where $N(a, b)$ denotes the Normal distribution with mean $a$ and standard deviation $b$ and $\Gamma(c, d)$ denotes the Gamma distribution with shape $c$ and scale $d$.

\section{Racing Diffusion Model (RDM)}

The RDM was composed of the 6 evidence-related parameters of the TRDM (i.e., parameters of the timing process were omitted, so subscript $E$ is also omitted for clarity), with the same scaling parameter setup for the mean evidence threshold. The parameter 
vector for participant $i$ was $\theta_{i} \in\left\{\gamma_{\mu}, \gamma_{\zeta}, \alpha_{\mu}, \alpha_{\zeta}, \sigma, \tau\right\}$. The RDM was defined as:

Data level :

$\left(R T_{i}\right.$, response $\left._{i}\right) \sim R D M\left(\theta_{i}\right)$

Group level :

$$
\begin{aligned}
\log \left(\theta_{i}\right) & \sim N\left(\theta^{\mu}, \theta^{\sigma}\right), \quad \forall \theta_{i} \in\left\{\theta_{i}\right\} \backslash\left\{\gamma_{\zeta, i}, \alpha_{\zeta, i}\right\} \\
\gamma_{\zeta, i} & \sim N\left(\gamma_{\zeta}^{\mu}, \gamma_{\zeta}^{\sigma}\right) \\
\alpha_{\zeta, i} & \sim N\left(\alpha_{\zeta}^{\mu}, \alpha_{\zeta}^{\sigma}\right)
\end{aligned}
$$

Prior distributions :

$$
\begin{aligned}
\gamma_{\mu}^{\mu} & \sim N(.5,1) \\
\gamma_{\zeta}^{\mu} & \sim N(1,1) \\
\alpha_{\mu}^{\mu}, \alpha_{\zeta}^{\mu}, \tau^{\mu} & \sim N(0,1) \\
\sigma^{\mu} & \sim N(-.5,1) \\
\gamma_{\mu}^{\sigma}, \gamma_{\zeta}^{\sigma}, \alpha_{\mu}^{\sigma}, \alpha_{\zeta}^{\sigma}, \sigma^{\sigma}, \tau^{\sigma} & \sim \Gamma(1,1)
\end{aligned}
$$

\section{Linear Ballistic Accumulator (LBA)}

The LBA has 7 parameters in total. We parameterized the LBA in a way that mirrored the TRDM and RDM, which differs to the standard LBA parameterization. In our setup, the LBA had a mean drift rate $\left(\gamma_{\mu}\right)$, drift rate sensitivity $\left(\gamma_{\zeta}\right)$, mean response threshold $\left(\alpha_{\mu}\right)$, threshold bias $\left(\alpha_{\zeta}\right)$, non-decision time $(\tau)$, maximum value of the start point distribution $(\chi)$, and across-trial variability in drift rate $(s)$. As with the TRDM and RDM, the mean response threshold was set as the scaling parameter $\left(\alpha_{\mu}=1\right)$. The parameter vector for participant $i$ was $\theta_{i} \in\left\{\gamma_{\mu}, \gamma_{\zeta}, \alpha_{\mu}, \alpha_{\zeta}, \tau, \chi, s\right\}$. The LBA likelihood function of the rtdists $\mathrm{R}$ package (Singmann et al., 2019) was used for all estimation routines. The 
LBA was defined as:

$$
\begin{aligned}
\text { Data level: } & \\
\left(R T_{i}, \text { response }_{i}\right) & \sim \operatorname{LBA}\left(\theta_{i}\right) \\
\text { Group level : } & \\
\log \left(\theta_{i}\right) & \sim N\left(\theta^{\mu}, \theta^{\sigma}\right), \quad \forall \theta_{i} \in\left\{\theta_{i}\right\} \backslash\left\{\gamma_{\zeta, i}, \alpha_{\zeta, i}\right\} \\
\gamma_{\zeta, i} & \sim N\left(\gamma_{\zeta}^{\mu}, \gamma_{\zeta}^{\sigma}\right) \\
\alpha_{\zeta, i} & \sim N\left(\alpha_{\zeta}^{\mu}, \alpha_{\zeta}^{\sigma}\right)
\end{aligned}
$$

Prior distributions :

$$
\begin{aligned}
\gamma_{\mu}^{\mu} & \sim N(.5,1) \\
\gamma_{\zeta}^{\mu} & \sim N(1,1) \\
\alpha_{\mu}^{\mu}, \alpha_{\zeta}^{\mu}, \tau^{\mu}, \chi^{\mu} & \sim N(0,1) \\
s^{\mu} & \sim N(-.5,1) \\
\gamma_{\mu}^{\sigma}, \gamma_{\zeta}^{\sigma}, \alpha_{\mu}^{\sigma}, \alpha_{\zeta}^{\sigma}, \tau^{\sigma}, \chi^{\sigma}, s^{\sigma} & \sim \Gamma(1,1)
\end{aligned}
$$

\section{Diffusion Decision Model (DDM)}

The DDM has 8 parameters in total: mean drift rate $(v)$, boundary separation $(a)$, relative start point of evidence accumulation $(z)$, non-decision time $\left(t_{e r}\right)$, within-trial variability in drift $(s)$, and across-trial variability in drift rate $\left(s_{v}\right)$, start point $\left(s_{z}\right)$, and nondecision time $\left(s_{t}\right)$. The within-trial variability parameter was set as the scaling parameter $(s=1)$. The DDM likelihood function of the rtdists $\mathrm{R}$ package (Singmann et al., 2019) was used for all estimation routines. The parameter vector for participant $i$ was 
$\theta_{i} \in\left\{v, a, z, t_{e r}, s_{v}, s_{z}, s_{t}\right\}$. The DDM was defined as:

Data level :

$\left(R T_{i}\right.$, response $\left._{i}\right) \sim \operatorname{DDM}\left(\theta_{i}\right)$

Group level :

$$
\begin{aligned}
\log \left(\theta_{i}\right) & \sim N\left(\theta^{\mu}, \theta^{\sigma}\right), \quad \forall \theta_{i} \in\left\{\theta_{i}\right\} \backslash\left\{v_{i}, z_{i}\right\} \\
v_{i} & \sim N\left(v^{\mu}, v^{\sigma}\right) \\
\operatorname{probit}\left(z_{i}\right) & \sim N\left(z^{\mu}, z^{\sigma}\right)
\end{aligned}
$$

Prior distributions :

$$
\begin{aligned}
v^{\mu} & \sim N(3,3) \\
a^{\mu} & \sim N(1,1) \\
z^{\mu}, t_{e r}^{\mu}, s_{v}^{\mu} & \sim N(0,1) \\
s_{z}^{\mu}, s_{t}^{\mu} & \sim N(-1,1) \\
v^{\sigma}, a^{\sigma}, z^{\sigma}, t_{e r}^{\sigma}, s_{v}^{\sigma}, s_{z}^{\sigma}, s_{t}^{\sigma} & \sim \Gamma(1,1)
\end{aligned}
$$


Appendix B

Model Parameterization

This appendix outlines the parameterization of each model reported in the main text. All prior distributions were as outlined in Appendix A.

\section{Part 1: Information-Controlled Decisions}

We tested a family of parameterizations for each of the four models for the perceptual decision data set. We tested all model parameterizations formed by factorially crossing the following parameters to freely vary across the speed, neutral and accuracy emphasis conditions, or be constrained to a single value across conditions:

- TRDM: $\gamma_{E, \mu}, \gamma_{E, \zeta}, \alpha_{E, \mu}, \tau_{E}$ and $\gamma_{T}$. We omitted the null model where no parameters were free to vary across emphasis conditions, producing $2^{5}-1=31$ TRDMs.

- RDM and LBA: $\gamma_{\mu}, \gamma_{\zeta}$ and $\tau$, producing $2^{3}=8$ RDMs and 8 LBAs. We assumed that the evidence threshold $\alpha_{\mu}$ always varied across conditions.

- DDM: $v$ and $t_{e r}$, producing $2^{2}=4$ DDMs. We again assumed that boundary separation $a$ always varied across conditions.

We estimated a single value of the following parameters across conditions for all model parameterizations:

- TRDM: $\alpha_{E, \zeta}, \sigma_{E}, \sigma_{T}$.

- RDM: $\alpha_{\zeta}, \sigma$.

- LBA: $\alpha_{\zeta}, \chi$, separate $s$ for correct and error drift rates.

- DDM: $z, s_{v}, s_{z}, s_{t}$.

The models for the lexical decisions were parameterized as for the perceptual decisions with the following two exceptions: (1) there were only speed and accuracy emphasis conditions (no neutral emphasis condition), and (2) there was a separate drift rate sensitivity parameter $\left(\alpha_{E, \zeta}, \alpha_{\zeta}\right.$ or $v$, depending on the model) for each word frequency condition (HF, LF, VLF, NW).

\section{Part 1: Time-Controlled Decisions}

The models for the explicit and implicit deadlines data sets were parameterized with a separate evidence sensitivity parameter for each coherence level: TRDM $-\gamma_{E, \zeta}, \mathrm{RDM}$ and LBA $-\gamma_{\zeta}$, and DDM $-v$. All other parameters were estimated as a single value across coherence conditions. The one exception was that the LBA estimated separate $s$ for correct and error drift rates.

The implicit deadline data contained just two subjects so we estimated parameters independently for each subject (i.e., without a hierarchy). The prior distributions for the subject-level parameters were set to the same values as the population mean prior distributions (i.e., $\theta^{\mu}$ ) reported in Appendix A. 


\section{Part 2: Timing-Based Guessing Policies}

For majority word and majority non-word conditions, we estimated separate values of $\alpha_{E, \mu}$ and $\alpha_{E, \zeta}$. As with the lexical decisions in Part 1, there was a separate $\gamma_{E, \zeta}$ for each word frequency condition (HF, LF, VLF, NW). All remaining parameters were estimated as a single value across conditions. For the TRDM guessing variant with a biased guessing probability, $g$, that is freely estimated from data we assumed the prior distribution

$$
\begin{aligned}
\text { Group level: } & \\
\operatorname{probit}\left(g_{i}\right) & \sim N\left(g^{\mu}, g^{\sigma}\right) \\
\text { Prior distributions : } & \\
g^{\mu} & \sim N(0,1) \\
g^{\sigma} & \sim \Gamma(1,1) .
\end{aligned}
$$

\section{Part 3: Pre-Emptive Responses}

The TRDM was estimated from the go-nogo data with a different parameterization for the evidence drift rates, instead using the parameterization of Hawkins et al. (2019). There were separate evidence rates for go responses to go stimuli $\left(\gamma_{E, g o \mid g o}\right)$, go responses to nogo stimuli $\left(\gamma_{E, g o \mid n o g o}\right)$ and nogo responses to nogo stimuli $\left(\gamma_{E, \text { nogo|nogo }}\right)$, estimated with the prior distributions

$$
\begin{aligned}
& \text { Group level: } \\
& \log \left(\gamma_{E, g o \mid g o, i}\right) \sim N\left(\gamma_{E, g o \mid g o}^{\mu}, \gamma_{E, g o \mid g o}^{\sigma}\right) \\
& \log \left(\gamma_{E, \text { go } \mid \text { nogo }, i}\right) \sim N\left(\gamma_{E, \text { go|nogo }}^{\mu}, \gamma_{E, \text { go|nogo }}^{\sigma}\right) \\
& \log \left(\gamma_{E, \text { nogo } \mid \text { nogo }, i}\right) \sim N\left(\gamma_{E, \text { nogo|nogo }}^{\mu}, \gamma_{E, \text { nogo|nogo }}^{\sigma}\right) \\
& \text { Prior distributions : } \\
& \gamma_{E, \text { go|go }}^{\mu}, \gamma_{E, \text { go|nogo }}^{\mu}, \gamma_{E, \text { nogo } \mid \text { nogo }}^{\mu} \sim N(1,1) \\
& \gamma_{E, g o \mid g o}^{\sigma}, \gamma_{E, g o \mid \text { nogo }}^{\sigma}, \gamma_{E, \text { nogo } \mid \text { nogo }}^{\sigma} \sim \Gamma(1,1) \text {. }
\end{aligned}
$$

There were so few nogo responses to go stimuli we made the simplifying assumption that $\gamma_{E, \text { nogo|go }}=0$, following Hawkins et al. (2019). The mean evidence threshold was set as the scaling parameter $\left(\alpha_{E, \mu}=1\right.$, cf. Appendix A) and because one of the response outcomes is unobserved we cannot estimate threshold bias, so we set $\alpha_{E, \zeta}=0$. The remaining parameters were estimated as standard and with the prior distributions specified in Appendix A.

\section{Part 3: Individual Differences in Timing Precision}

The TRDM was simultaneously estimated from data in the choice and interval production tasks. In the choice task, we freely estimated separate values of the following 
parameters across the speed and accuracy emphasis conditions: $\gamma_{E, \mu}, \gamma_{E, \zeta}, \alpha_{E, \mu}, \tau_{E}$ and $\gamma_{T}$, which mirrors the free parameters estimated in the information-controlled tasks in Part 1. We estimated a single value of the remaining TRDM parameters from the choice task. We independently estimated $\gamma_{T}$ and $\sigma_{T}$ from the interval production task. 
Appendix C

Complete Model Comparison Results

Tables $\mathrm{C} 1$ and $\mathrm{C} 2$ show the results of the model comparison between the set of 51 models from the perceptual and lexical data sets reported in Part 1 of the paper. Tables $\mathrm{C} 1$ and $\mathrm{C} 2$ are summarized into Tables 1 and 2 of the main text by showing only the best performing (i.e., smallest DIC) parameterization of each of the four model classes under consideration (TRDM, RDM, LBA, DDM). 
Table C1

DIC model comparison for perceptual decisions (Forstmann et al., 2008). Rows show models. Columns 2-6 indicate whether a parameter was freely estimated across the levels of the time pressure manipulation $(\checkmark)$ or constrained to a single value (blank; - indicates a parameter does not exist in a model). Column 7 shows DICs zero-referenced to the DIC-preferred model such that positive values indicate a poorer explanation of the data. Rows are ordered by DIC. Colors added to aid interpretation, where lighter and darker shades of blue show TRDMs that freely estimate or constrain evidence thresholds, respectively.

\begin{tabular}{|c|c|c|c|c|c|c|}
\hline Model & $\begin{array}{l}\text { Evidence } \\
\text { Threshold }\end{array}$ & $\begin{array}{l}\text { Evidence } \\
\text { Rate }\end{array}$ & $\begin{array}{l}\text { Evidence } \\
\text { Sensitivity }\end{array}$ & $\begin{array}{l}\text { Evidence } \\
\text { NDT }\end{array}$ & $\begin{array}{l}\text { Timing } \\
\text { Rate }\end{array}$ & $\begin{array}{c}\text { Perceptual Decisions } \\
\Delta \text { DIC }\end{array}$ \\
\hline TRDM & $\checkmark$ & $\checkmark$ & & $\checkmark$ & $\checkmark$ & 0 \\
\hline TRDM & $\checkmark$ & & $\checkmark$ & $\checkmark$ & $\checkmark$ & 40 \\
\hline TRDM & $\checkmark$ & $\checkmark$ & $\checkmark$ & $\checkmark$ & $\checkmark$ & 79 \\
\hline TRDM & $\checkmark$ & $\checkmark$ & & & $\checkmark$ & 89 \\
\hline TRDM & & $\checkmark$ & $\checkmark$ & $\checkmark$ & $\checkmark$ & 118 \\
\hline DDM & $\checkmark$ & - & & $\checkmark$ & - & 118 \\
\hline TRDM & $\checkmark$ & $\checkmark$ & $\checkmark$ & & $\checkmark$ & 127 \\
\hline DDM & $\checkmark$ & - & $\checkmark$ & $\checkmark$ & - & 138 \\
\hline TRDM & & & $\checkmark$ & $\checkmark$ & $\checkmark$ & 140 \\
\hline TRDM & $\checkmark$ & & & & $\checkmark$ & 141 \\
\hline TRDM & $\checkmark$ & $\checkmark$ & $\checkmark$ & $\checkmark$ & & 151 \\
\hline TRDM & & $\checkmark$ & & $\checkmark$ & $\checkmark$ & 154 \\
\hline TRDM & $\checkmark$ & & & $\checkmark$ & $\checkmark$ & 199 \\
\hline TRDM & & $\checkmark$ & $\checkmark$ & $\checkmark$ & & 200 \\
\hline TRDM & $\checkmark$ & & $\checkmark$ & & $\checkmark$ & 214 \\
\hline TRDM & $\checkmark$ & $\checkmark$ & & $\checkmark$ & & 238 \\
\hline TRDM & & $\checkmark$ & & $\checkmark$ & & 306 \\
\hline TRDM & & & & $\checkmark$ & $\checkmark$ & 316 \\
\hline TRDM & $\checkmark$ & & $\checkmark$ & $\checkmark$ & & 334 \\
\hline TRDM & $\checkmark$ & $\checkmark$ & $\checkmark$ & & & 358 \\
\hline TRDM & $\checkmark$ & & $\checkmark$ & & & 428 \\
\hline TRDM & $\checkmark$ & $\checkmark$ & & & & 457 \\
\hline LBA & $\checkmark$ & $\checkmark$ & $\checkmark$ & & - & 472 \\
\hline LBA & $\checkmark$ & $\checkmark$ & $\checkmark$ & $\checkmark$ & - & 495 \\
\hline TRDM & $\checkmark$ & & & $\checkmark$ & & 502 \\
\hline LBA & $\checkmark$ & & $\checkmark$ & $\checkmark$ & - & 507 \\
\hline TRDM & $\checkmark$ & & & & & 549 \\
\hline RDM & $\checkmark$ & $\checkmark$ & $\checkmark$ & $\checkmark$ & - & 621 \\
\hline LBA & $\checkmark$ & & $\checkmark$ & & - & 658 \\
\hline DDM & $\checkmark$ & - & & & - & 662 \\
\hline TRDM & & $\checkmark$ & $\checkmark$ & & $\checkmark$ & 685 \\
\hline DDM & $\checkmark$ & - & $\checkmark$ & & - & 687 \\
\hline RDM & $\checkmark$ & $\checkmark$ & $\checkmark$ & & - & 708 \\
\hline RDM & $\checkmark$ & $\checkmark$ & & $\checkmark$ & - & 712 \\
\hline LBA & $\checkmark$ & $\checkmark$ & & $\checkmark$ & - & 747 \\
\hline RDM & $\checkmark$ & $\checkmark$ & & & - & 751 \\
\hline RDM & $\checkmark$ & & $\checkmark$ & $\checkmark$ & - & 771 \\
\hline RDM & $\checkmark$ & & $\checkmark$ & & - & 782 \\
\hline TRDM & & $\checkmark$ & & & $\checkmark$ & 797 \\
\hline RDM & $\checkmark$ & & & $\checkmark$ & - & 813 \\
\hline RDM & $\checkmark$ & & & & - & 819 \\
\hline TRDM & & & $\checkmark$ & & $\checkmark$ & 823 \\
\hline LBA & $\checkmark$ & & & $\checkmark$ & - & 858 \\
\hline LBA & $\checkmark$ & $\checkmark$ & & & - & 886 \\
\hline LBA & $\checkmark$ & & & & - & 903 \\
\hline TRDM & & & $\checkmark$ & $\checkmark$ & & 1,166 \\
\hline TRDM & & & & $\checkmark$ & & 1,278 \\
\hline TRDM & & & & & $\checkmark$ & 1,340 \\
\hline TRDM & & $\checkmark$ & & & & 1,379 \\
\hline TRDM & & $\checkmark$ & $\checkmark$ & & & 1,421 \\
\hline TRDM & & & $\checkmark$ & & & 2,719 \\
\hline
\end{tabular}


Table C2

DIC model comparison for lexical decisions (Wagenmakers et al., 2008). All details are as described in Table C1.

\begin{tabular}{|c|c|c|c|c|c|c|}
\hline Model & $\begin{array}{l}\text { Evidence } \\
\text { Threshold }\end{array}$ & $\begin{array}{c}\text { Evidence } \\
\text { Rate }\end{array}$ & $\begin{array}{l}\text { Evidence } \\
\text { Sensitivity }\end{array}$ & $\begin{array}{l}\text { Evidence } \\
\text { NDT }\end{array}$ & $\begin{array}{l}\text { Timing } \\
\text { Rate }\end{array}$ & $\begin{array}{c}\text { Lexical Decisions } \\
\Delta \text { DIC }\end{array}$ \\
\hline TRDM & $\checkmark$ & $\checkmark$ & $\checkmark$ & $\checkmark$ & $\checkmark$ & 0 \\
\hline TRDM & & $\checkmark$ & $\checkmark$ & $\checkmark$ & $\checkmark$ & 29 \\
\hline TRDM & $\checkmark$ & $\checkmark$ & & $\checkmark$ & $\checkmark$ & 146 \\
\hline LBA & $\checkmark$ & $\checkmark$ & $\checkmark$ & $\checkmark$ & - & 175 \\
\hline TRDM & $\checkmark$ & & $\checkmark$ & $\checkmark$ & $\checkmark$ & 189 \\
\hline TRDM & $\checkmark$ & $\checkmark$ & $\checkmark$ & & $\checkmark$ & 191 \\
\hline TRDM & & $\checkmark$ & & $\checkmark$ & $\checkmark$ & 203 \\
\hline TRDM & & & $\checkmark$ & $\checkmark$ & $\checkmark$ & 225 \\
\hline LBA & $\checkmark$ & $\checkmark$ & $\checkmark$ & & - & 250 \\
\hline TRDM & $\checkmark$ & & $\checkmark$ & & $\checkmark$ & 305 \\
\hline TRDM & $\checkmark$ & $\checkmark$ & & & $\checkmark$ & 357 \\
\hline TRDM & $\checkmark$ & $\checkmark$ & $\checkmark$ & $\checkmark$ & & 539 \\
\hline TRDM & & $\checkmark$ & $\checkmark$ & $\checkmark$ & & 550 \\
\hline TRDM & $\checkmark$ & & & $\checkmark$ & $\checkmark$ & 586 \\
\hline TRDM & $\checkmark$ & & & & $\checkmark$ & 695 \\
\hline TRDM & $\checkmark$ & $\checkmark$ & & $\checkmark$ & & 803 \\
\hline TRDM & & $\checkmark$ & & $\checkmark$ & & 880 \\
\hline LBA & $\checkmark$ & & $\checkmark$ & $\checkmark$ & - & 982 \\
\hline DDM & $\checkmark$ & - & $\checkmark$ & $\checkmark$ & - & 1,022 \\
\hline TRDM & $\checkmark$ & $\checkmark$ & $\checkmark$ & & & 1,054 \\
\hline TRDM & & $\checkmark$ & $\checkmark$ & & $\checkmark$ & 1,147 \\
\hline DDM & $\checkmark$ & - & & $\checkmark$ & - & 1,275 \\
\hline TRDM & $\checkmark$ & $\checkmark$ & & & & 1,308 \\
\hline TRDM & & & $\checkmark$ & & $\checkmark$ & 1,339 \\
\hline TRDM & & $\checkmark$ & & & $\checkmark$ & 1,451 \\
\hline LBA & $\checkmark$ & $\checkmark$ & & $\checkmark$ & - & 1,467 \\
\hline LBA & $\checkmark$ & & & $\checkmark$ & - & 1,598 \\
\hline LBA & $\checkmark$ & & $\checkmark$ & & - & 1,619 \\
\hline LBA & $\checkmark$ & $\checkmark$ & & & - & 1,646 \\
\hline DDM & $\checkmark$ & - & $\checkmark$ & & - & 1,729 \\
\hline TRDM & $\checkmark$ & & $\checkmark$ & & & 1,844 \\
\hline DDM & $\checkmark$ & - & & & - & 1,872 \\
\hline TRDM & $\checkmark$ & 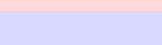 & $\checkmark$ & $\checkmark$ & & 1,987 \\
\hline LBA & $\checkmark$ & & & & - & 2,062 \\
\hline TRDM & & $\checkmark$ & $\checkmark$ & & & 2,114 \\
\hline TRDM & & $\checkmark$ & & & & 2,353 \\
\hline RDM & $\checkmark$ & $\checkmark$ & $\checkmark$ & $\checkmark$ & - & 2,358 \\
\hline TRDM & & & & $\checkmark$ & $\checkmark$ & 2,372 \\
\hline TRDM & $\checkmark$ & & & $\checkmark$ & & 2,546 \\
\hline TRDM & $\checkmark$ & & & & & 2,589 \\
\hline RDM & $\checkmark$ & $\checkmark$ & $\checkmark$ & & - & 2,620 \\
\hline RDM & $\checkmark$ & $\checkmark$ & & $\checkmark$ & - & 2,805 \\
\hline RDM & $\checkmark$ & $\checkmark$ & & & - & 3,024 \\
\hline TRDM & & & $\checkmark$ & $\checkmark$ & & 3,086 \\
\hline RDM & $\checkmark$ & & $\checkmark$ & $\checkmark$ & - & 3,551 \\
\hline RDM & $\checkmark$ & & $\checkmark$ & & - & 3,816 \\
\hline RDM & $\checkmark$ & & & $\checkmark$ & - & 3,950 \\
\hline RDM & $\checkmark$ & & & & - & 4,200 \\
\hline TRDM & & & & & $\checkmark$ & 4,852 \\
\hline TRDM & & & & $\checkmark$ & & 5,013 \\
\hline TRDM & & & $\checkmark$ & & & 5,233 \\
\hline
\end{tabular}




\section{Appendix D \\ Parameter Recovery Method}

We performed a parameter recovery exercise for each data set to confirm that the parameters of the TRDM can be reliably recovered from data. We started with the TRDM parameters estimated from the DIC-best model in each data set. We randomly sampled a parameter vector from each participant's posterior distribution of the TRDM parameters and used them to generate synthetic data with the same properties as the real data (i.e., number of trials for each stimulus and emphasis condition, total number of trials, number of participants). We then estimated the TRDM from these simulated data using identical methods as those applied to the real data (Appendix A). We independently repeated the procedure 10 times. This produced 10 independent hierarchical estimation exercises that assessed parameter recovery from a total of $10 \times S$ participant-level posterior distributions, where $S$ is the number of participants in each data set. The focus of the recovery analysis is at the participant level.

We quantify parameter recovery in terms of summary statistics of the difference distribution between the median of the participant-level posterior distributions estimated from the simulated data and the data-generating parameters. These summary statistics are shown in Tables 3, 4, 6, 7, 9, 11 and 12 of the main text. Perfect recovery produces a distribution centred at 0 . There is good coverage indicated when the $95 \%$ credible interval contains the data-generating values (i.e., when it contains 0 ). Positive values indicate that the recovered parameters are over-estimates of the data-generating parameters and negative values indicate that recovered parameters are under-estimates of the data-generating parameters. In all cases, the difference distributions were centered at or near 0 for all TRDM parameters. This indicates good parameter recovery: the parameters of the TRDM can be recovered in typically-sized data sets and thus it is safe to interpret the parameter estimates. 
Appendix E

Parameter Effects For Information-Controlled Decisions

Tables E1 and E2 show estimates of the standardized effect size $(z)$ of the difference between the speed and accuracy conditions of the perceptual and lexical decisions from Part 1 of the paper. We follow Strickland et al.'s (2018) approach to calculate these measures. Separately for each posterior sampling iteration, we take the difference between each participant's parameter estimates in the speed and accuracy conditions and then average the differences over participants. Across iterates, this forms the posterior distribution of the across-participant average difference between the speed and accuracy conditions for each parameter. Tables E1 and E2 summarize these distributions with the median and 95\% credible interval. We also use these distributions to estimate the standardized effect size as the mean over the distribution of average differences divided by its standard deviation. 
Table E1

Parameter effects and standardized effect sizes for the speed-accuracy tradeoff manipulation in the perceptual decision data (Forstmann et al., 2008). Effects are shown for the DIC-best parameterization for each of the four models from the main text (cf. Table 1). Effects are calculated as the average participant-level difference between the speed and accuracy conditions as a function of the parameters that were free to vary over this factor. Column 3 shows the median of the posterior distribution of the across-participant average difference between the speed and accuracy conditions, with underset values showing $95 \%$ credible interval in parentheses. Column 4 shows the standardized effect size of the difference between speed and accuracy. Negative values indicate the speed condition has a lower value of the parameter than the accuracy condition, and positive values indicate the speed condition has a higher value of the parameter than the accuracy condition. See Appendix E for details.

\begin{tabular}{|c|c|c|c|}
\hline Model & Parameter & $\begin{array}{c}\text { Posterior Median } \\
(95 \% \mathrm{CI})\end{array}$ & $\begin{array}{l}\text { Standardized } \\
\text { Effect Size }(z)\end{array}$ \\
\hline \multirow{4}{*}{ TRDM } & $\underset{\left(\alpha_{E, \mu}\right)}{\text { Evidence Threshold }}$ & $\begin{array}{c}-.09 \\
(-.14,-.04)\end{array}$ & -3.5 \\
\hline & $\underset{\left(\gamma_{E, \mu}\right)}{\text { Evidence Rate }}$ &. .63 & 5.9 \\
\hline & $\underset{\left(\tau_{E}\right)}{\text { Non-decision time }}$ & $\begin{array}{c}-.05 \\
(-.06,-.04)\end{array}$ & -14.1 \\
\hline & $\underset{\left(\gamma_{T}\right)}{\operatorname{Timer} \text { Rate }}$ & $\begin{array}{c}.87 \\
(.74,1.01)\end{array}$ & 13.1 \\
\hline \multirow{2}{*}{ DDM } & $\begin{array}{c}\text { Boundary Separation } \\
\qquad(a)\end{array}$ & $\begin{array}{c}-.36 \\
(-.39,-.33)\end{array}$ & -25.7 \\
\hline & $\underset{(\tau)}{\text { Non-decision time }}$ & $\begin{array}{c}-.04 \\
(-.04,-.03)\end{array}$ & -21.2 \\
\hline \multirow{3}{*}{ LBA } & $\underset{\left(\alpha_{\mu}\right)}{\text { Threshold }}$ & $\begin{array}{c}-.27 \\
(-.30,-.24)\end{array}$ & -17.4 \\
\hline & $\underset{\left(\gamma_{\mu}\right)}{\text { Evidence Rate }}$ & $\begin{array}{c}.95 \\
(.82,1.10)\end{array}$ & 13.6 \\
\hline & $\begin{array}{c}\text { Evidence Sensitivity } \\
\left(\gamma_{\zeta}\right)\end{array}$ & $\begin{array}{c}-1.46 \\
(-1.66,-1.28) \\
\end{array}$ & -14.9 \\
\hline \multirow{4}{*}{ RDM } & $\underset{\left(\alpha_{\mu}\right)}{\text { Threshold }}$ & $\begin{array}{c}-.22 \\
(-.27,-.17)\end{array}$ & -8.6 \\
\hline & $\underset{\left(\gamma_{\mu}\right)}{\operatorname{Evidence} \text { Rate }}$ & $\begin{array}{c}.86 \\
(.73, .98)\end{array}$ & 13.4 \\
\hline & $\begin{array}{c}\text { Evidence Sensitivity } \\
\left(\gamma_{\zeta}\right)\end{array}$ & $\begin{array}{c}-.72 \\
(-.88,-.56)\end{array}$ & -8.6 \\
\hline & $\underset{(\tau)}{\text { Non-decision time }}$ & $\begin{array}{c}-.03 \\
(-.04,-.03)\end{array}$ & -9.8 \\
\hline
\end{tabular}


Table E2

Parameter effects and standardized effect sizes for the speed-accuracy tradeoff and word frequency manipulations in the lexical decision data (Wagenmakers et al., 2008). Effects are shown for the DIC-best parameterization for each of the four models from the main text (cf. Table 2). All other details are as described in Table E1.

\begin{tabular}{|c|c|c|c|c|}
\hline Model & Parameter & Stimulus & $\begin{array}{l}\text { Posterior Median } \\
\qquad(95 \% \mathrm{CI})\end{array}$ & $\begin{array}{l}\text { Standardized } \\
\text { Effect Size }(z)\end{array}$ \\
\hline \multirow{8}{*}{ TRDM } & $\underset{\left(\alpha_{E, \mu}\right)}{\operatorname{Evidence} \text { Threshold }}$ & - & $\begin{array}{c}-.08 \\
(-.12,-.04)\end{array}$ & -4.1 \\
\hline & $\underset{\left(\gamma_{E, \mu}\right)}{\text { Evidence Rate }}$ & - & $(.64, .80)$ & 17.3 \\
\hline & \multirow{4}{*}{$\begin{array}{l}\text { Evidence Sensitivity } \\
\qquad\left(\gamma_{E, \zeta}\right)\end{array}$} & $\mathrm{HF}$ & $\begin{array}{c}.74 \\
(.44,1.04)\end{array}$ & 4.82 \\
\hline & & $\mathrm{LF}$ & $(-.16, .31)$ & .65 \\
\hline & & VLF & $\begin{array}{c}-.09 \\
(-.29, .11)\end{array}$ & -.88 \\
\hline & & NW & $\begin{array}{c}1.06 \\
(.90,1.23)\end{array}$ & 12.7 \\
\hline & $\underset{\left(\tau_{E}\right)}{\text { Non-decision time }}$ & - & $\begin{array}{c}-.05 \\
(-.05,-.04)\end{array}$ & -14.8 \\
\hline & $\underset{\left(\gamma_{T}\right)}{\underset{1}{\operatorname{Timer}} \text { Rate }}$ & - & $\begin{array}{c}.87 \\
(.82, .92) \\
\end{array}$ & 32.4 \\
\hline \multirow{7}{*}{ LBA } & $\underset{\left(\alpha_{\mu}\right)}{\text { Threshold }}$ & - & $\begin{array}{c}.08 \\
(.04, .12)\end{array}$ & 3.9 \\
\hline & $\underset{\left(\gamma_{\mu}\right)}{\operatorname{Evidence} \text { Rate }}$ & - & $\begin{array}{c}1.40 \\
(1.34,1.46)\end{array}$ & 47.7 \\
\hline & \multirow{4}{*}{$\underset{\left(\gamma_{\zeta}\right)}{\text { Evidence Sensitivity }}$} & $\mathrm{HF}$ & $\begin{array}{c}-1.76 \\
(-1.84,-1.68)\end{array}$ & -41.8 \\
\hline & & $\mathrm{LF}$ & $\begin{array}{c}-1.71 \\
(-1.79,-1.63)\end{array}$ & -44.4 \\
\hline & & VLF & $\begin{array}{c}-1.63 \\
(-1.71,-1.55)\end{array}$ & -41.7 \\
\hline & & NW & $\begin{array}{c}-1.48 \\
(-1.54,-1.41)\end{array}$ & -44.0 \\
\hline & $\underset{(\tau)}{\text { Non-decision time }}$ & - & $\begin{array}{l}-.05 \\
(-.06,-.04)\end{array}$ & -9.7 \\
\hline \multirow{6}{*}{ DDM } & $\underset{(a)}{\text { Boundary Separation }}$ & - & $\begin{array}{c}-.67 \\
(-.70,-.64)\end{array}$ & -45.3 \\
\hline & \multirow{4}{*}{$\underset{(v)}{\operatorname{Drift}}$ Rate } & $\mathrm{HF}$ & $\begin{array}{c}.10 \\
(-.10, .29)\end{array}$ & .94 \\
\hline & & $\mathrm{LF}$ & $\begin{array}{c}-.49 \\
(-.65,-.34)\end{array}$ & -6.4 \\
\hline & & VLF & $\begin{array}{c}-.60 \\
(-.74,-.46)\end{array}$ & -8.5 \\
\hline & & NW & $\begin{array}{c}-.07 \\
(-.17, .03)\end{array}$ & -1.36 \\
\hline & $\underset{(\tau)}{\text { Non-decision time }}$ & - & $\begin{array}{c}-.04 \\
(-.04,-.04)\end{array}$ & -24.5 \\
\hline \multirow{7}{*}{$\mathrm{RDM}$} & $\underset{\left(\alpha_{\mu}\right)}{\text { Threshold }}$ & - & $\begin{array}{c}-.04 \\
(-.08,-.01)\end{array}$ & -2.6 \\
\hline & $\underset{\left(\gamma_{\mu}\right)}{\operatorname{Evidence}}$ Rate & - & $\begin{array}{c}.88 \\
(.83, .92)\end{array}$ & 37.1 \\
\hline & \multirow{4}{*}{$\underset{\left(\gamma_{\zeta}\right)}{\text { Evidence Sensitivity }}$} & $\mathrm{HF}$ & $\begin{array}{c}-.04 \\
(-.19, .10)\end{array}$ & -.6 \\
\hline & & $\mathrm{LF}$ & $\begin{array}{c}-.45 \\
(-.57,-.33)\end{array}$ & -7.3 \\
\hline & & VLF & $\begin{array}{c}-.53 \\
(-.65,-.42)\end{array}$ & -9.0 \\
\hline & & NW & $\begin{array}{c}.09 \\
(.01, .17)\end{array}$ & 2.1 \\
\hline & $\underset{(\tau)}{\text { Non-decision time }}$ & & $\begin{array}{c}-.06 \\
(-.06,-.05)\end{array}$ & -14.3 \\
\hline
\end{tabular}

